\title{
Photo-mediated Decarboxylative Giese-Type Reaction using Organic Pyrimidopteridine Photoredox Catalysts
}

\author{
Firas El-Hage, Christopher Schöll and Jola Pospech* \\ Leibniz Institute for Catalysis, Albert-Einstein-Straße 29a, 18057 Rostock. \\ Jola.Pospech@catalysis.de
}

\section{Supporting Information}

\section{Contents}

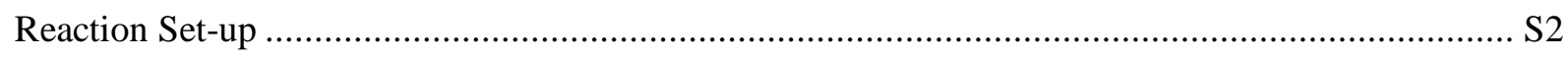

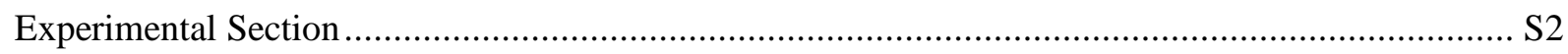

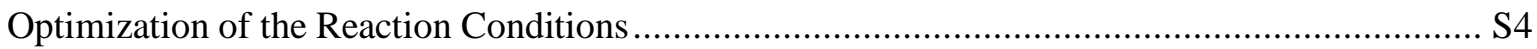

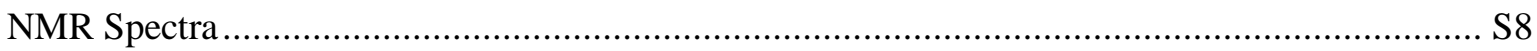

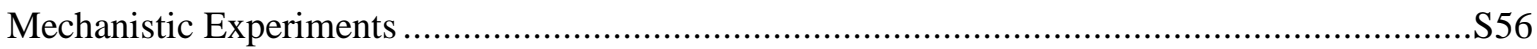

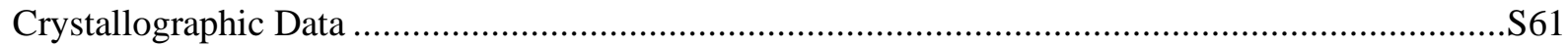

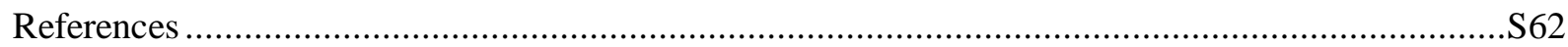




\section{Reaction Set-up}

Unless otherwise stated, all reactions were performed on a $0.5 \mathrm{mmol}$ scale and a molarity of $0.1 \mathrm{M}$ under inert atmosphere of argon in a $5 \mathrm{ml}$ microwave vessel (Biotage $\left.{ }^{\circledR}\right)$. The reactions were irradiated using 2 x $30 \mathrm{~W}$ UV-lamp ONFURO IP66 in 5.7 and $10.4 \mathrm{~cm}$ distance (Figure 1). The emission spectra of the light source and the superimposed emission spectra of the light source and the absorption spectra of the PrPPT (5b) and PrPPTNO (4b) catalyst are shown in Figure 2.
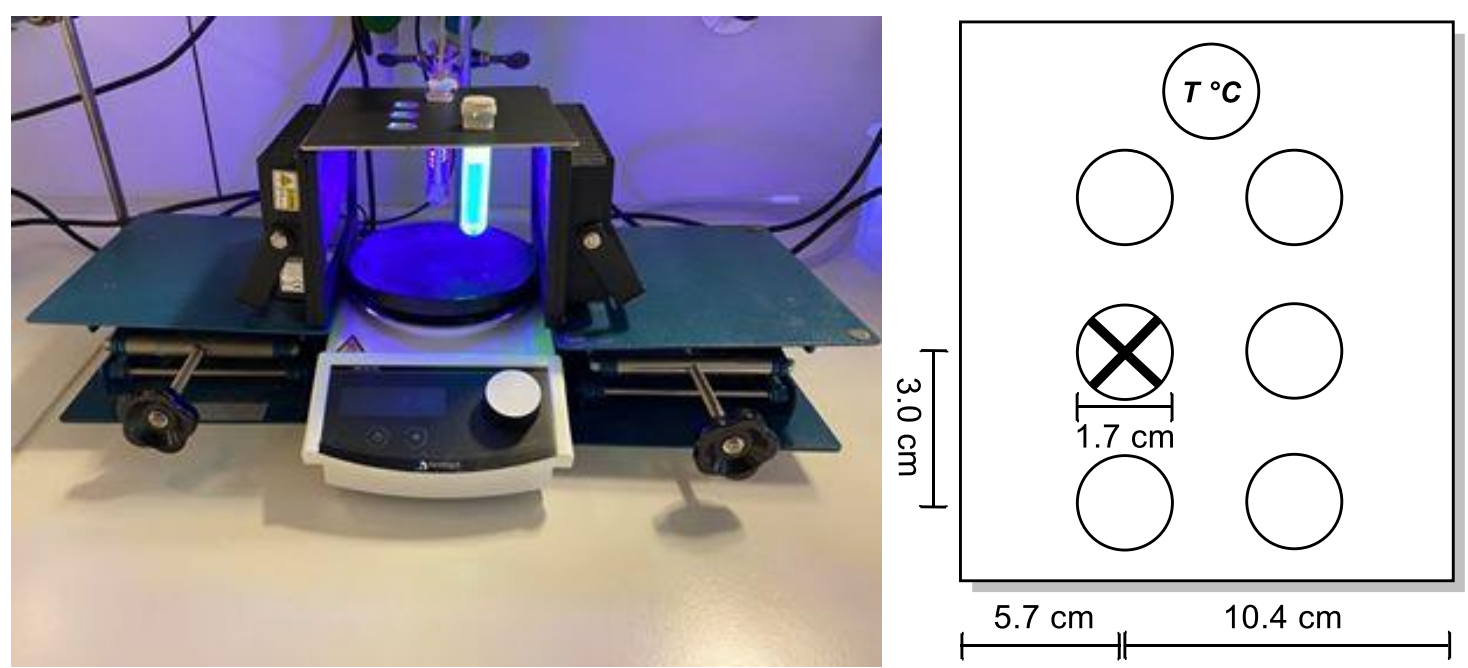

Figure S1. Reaction set-up.
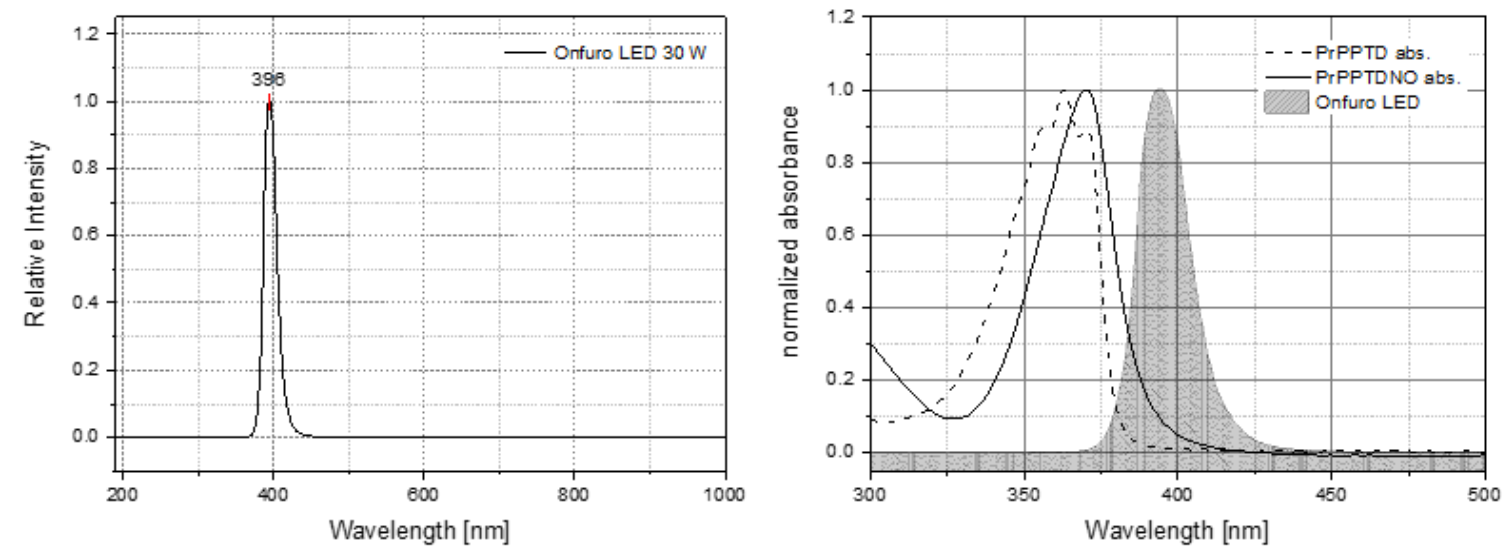

Figure S2. Emission spectrum of the utilized UV-lamp ONFURO IP66 (30 W). Absorption spectra of PrPPTNO (4b) and PrPPT (5b) and emission spectra of ONFURO IP66 LED. 
Figure S3. Photocatalysts.

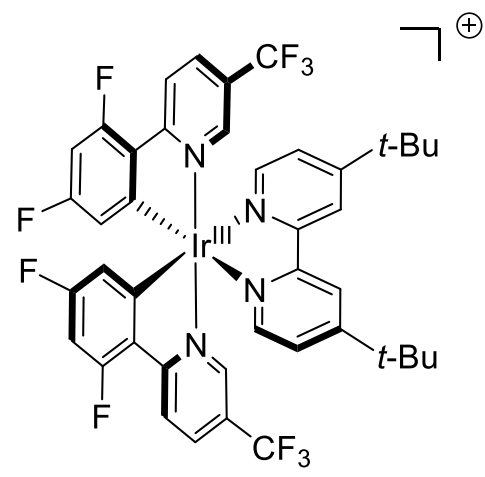

$\operatorname{Ir}\left[\mathrm{dF}\left(\mathrm{CF}_{3}\right) \mathrm{ppy}\right]_{2}\left(\mathrm{dtbbpy}^{+}\right.$

(1)<smiles></smiles>

Eosin $Y$

(2)<smiles></smiles>

[Acr-Mes] ${ }^{+}$

(3)
$E_{1 / 2}\left(P C^{*} / P C_{-}\right)=+1.32 \mathrm{~V}$
$\mathrm{E}_{1 / 2}\left(\mathrm{PC}^{*} / \mathrm{PC}^{--}\right)=+0.83 \mathrm{~V}$
$E_{1 / 2}\left(P C^{*} / P C^{-}\right)=+2.06 \mathrm{~V}$
$E_{1 / 2}(P C / P C)=-1.37 \mathrm{~V}$
$E_{1 / 2}\left(P C / P C^{-}\right)=-1.14 \mathrm{~V}$

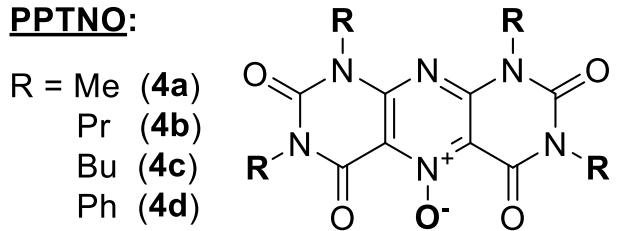

Pyrimidopteridine $\mathbf{N}$-oxide (PPTNO)<smiles>[R]n1c(=O)c2nc3c(=O)n([R])c(=O)n([R])c3nc2n([R])c1=O</smiles>

Pyrimidopteridine (PPT)
PPT:

$R=\operatorname{Me}(5 a)$

$\operatorname{Pr}(5 \mathbf{b})$

$\mathrm{Bu}(5 \mathrm{c})$

$\mathrm{Ph}(5 \mathrm{~d})$ 


\section{Experimental Section}

\section{General procedure A: Photocatalytic Giese-type addition}

Reactions were performed under Schlenk conditions using a $5 \mathrm{ml}$ process vial equipped with a NS 14.5 rubber septum. To this vial was added the appropriate carboxylic acid $(0.50 \mathrm{mmol}$, 1.0 equiv.), photocatalyst PrPPTNO (5.0 mol- $\%$ ) and $\mathrm{K}_{3} \mathrm{PO}_{4}$ (20 mol-\%). Next, the vial was evacuated and purged with argon for 3 times. The corresponding olefin $(1.0 \mathrm{mmol}, 2.0$ equiv.) was then added to the flask under argon. Afterwards, acetonitrile $(4.5 \mathrm{~mL})$ and degassed water $(0.5 \mathrm{ml})$ were added and the reaction mixture was stirred for 2 minutes in the dark. The reaction mixture was then irradiated for the indicated time and quenched afterwards upon exposure to air and bubbling air through the solution using a pipette. The reaction was concentrated under reduced pressure and the crude mixture was purified by column chromatography.

\section{Optimization of the Reaction Conditions}

Table S1. Photocatalysts screening with PPTNO- and PPT derivatives.

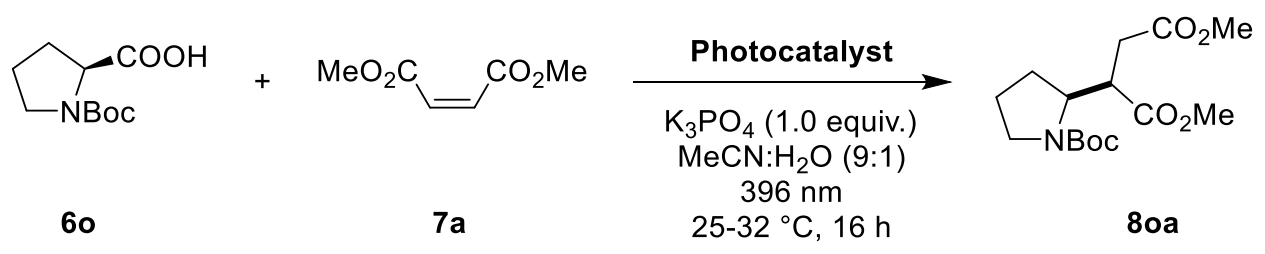

\begin{tabular}{|c|c|c|c|}
\hline Entry & Photocatalyst & Catalyst loading & Yield $^{[a]}$ \\
\hline 1 & PrPPTNO (4b) & $5.0 \mathrm{~mol}-\%$ & $96 \%$ (88\%) \\
\hline 2 & PrPPTN (5b) & $5.0 \mathrm{~mol}-\%$ & quant. (quant.) \\
\hline 3 & BUPPTNO (4c) & $5.0 \mathrm{~mol}-\%$ & $96 \%$ (87\%) \\
\hline 4 & BuPPTN (5c) & $5.0 \mathrm{~mol}-\%$ & $98 \%$ (91\%) \\
\hline 5 & MePPTNO (4a) & $5.0 \mathrm{~mol}-\%$ & $49 \%$ \\
\hline 6 & MePPTN (5a) & $5.0 \mathrm{~mol}-\%$ & $56 \%$ \\
\hline 7 & PhPPTNO (4d) & $5.0 \mathrm{~mol}-\%$ & $38 \%$ \\
\hline 8 & PhPPTN (5d) & $5.0 \mathrm{~mol}-\%$ & $43 \%$ \\
\hline
\end{tabular}


Table S2. Solvent Screening.

\begin{tabular}{|c|c|c|c|c|}
\hline & $y_{-\mathrm{NBOC}}^{\mathrm{COOH}}$ & $\mathrm{MeO}_{2} \mathrm{C} \_\mathrm{CO}_{2} \mathrm{Me}$ & $\begin{array}{c}\text { PrPPTNO (4b) } \\
5 \text { mol- } \% \\
\mathrm{~K}_{3} \mathrm{PO}_{4}(1.0 \text { eq.) } \\
\text { Solvent } \\
396 \mathrm{~nm} \\
25-32{ }^{\circ} \mathrm{C}, 16 \mathrm{~h}\end{array}$ & $\int_{80 a}^{\mathrm{CO}_{2} \mathrm{Me}}$ \\
\hline Entry & Solvent & Ratio & Molarity & Yield $^{[\mathrm{aj}]}$ \\
\hline 1 & $\mathrm{MeCN}: \mathrm{H}_{2} \mathrm{O}$ & 9:1 & $0.10 \mathrm{M}$ & $96 \%(88 \%)$ \\
\hline 2 & $\mathrm{MeCN}: \mathrm{H}_{2} \mathrm{O}$ & $7: 3$ & $0.10 \mathrm{M}$ & $73 \%$ \\
\hline 3 & $\mathrm{MeCN}$ & & $0.10 \mathrm{M}$ & $73 \%$ \\
\hline 4 & DCM & & $0.10 \mathrm{M}$ & $61 \%$ \\
\hline 5 & Acetone & & $0.10 \mathrm{M}$ & $58 \%$ \\
\hline 6 & DCE & & $0.10 \mathrm{M}$ & $40 \%$ \\
\hline 7 & iPrOH & & $0.10 \mathrm{M}$ & $17 \%$ \\
\hline 8 & THF & & $0.10 \mathrm{M}$ & $12 \%$ \\
\hline 9 & $\mathrm{H}_{2} \mathrm{O}$ & & $0.10 \mathrm{M}$ & $8 \%$ \\
\hline 10 & HFIP & & $0.10 \mathrm{M}$ & $8 \%$ \\
\hline 11 & DMSO & & $0.10 \mathrm{M}$ & $1 \%$ \\
\hline
\end{tabular}

[a] Yields were determined by GC using biphenyl as internal standard. Yields in parentheses represent isolated yields. 
Table S3. Variation of Catalyst loadings.

\begin{tabular}{|c|c|c|c|}
\hline $6 c$ & $+\mathrm{MeO}_{2} \mathrm{C}$ & 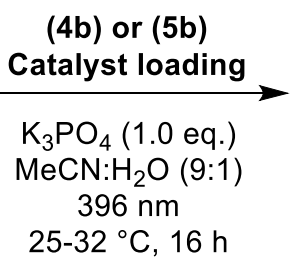 & $\mathrm{O}_{2} \mathrm{Me}$ \\
\hline Entry & Photocatalyst & Catalyst loading & Yield $^{[a]}$ \\
\hline 1 & PrPPTNO (4b) & $2.5 \mathrm{~mol}-\%$ & $58 \%$ \\
\hline 2 & PrPPTN (5b) & $2.5 \mathrm{~mol}-\%$ & $72 \%$ \\
\hline 3 & PrPPTNO (4b) & $1.0 \mathrm{~mol}-\%$ & $41 \%$ \\
\hline 4 & PrPPTN (5b) & $1.0 \mathrm{~mol}-\%$ & $41 \%$ \\
\hline 5 & PrPPTNO (4b) & $0.5 \mathrm{~mol}-\%$ & $18 \%$ \\
\hline 6 & PrPPTN (5b) & $0.5 \mathrm{~mol}-\%$ & $28 \%$ \\
\hline
\end{tabular}

${ }^{\text {[a] }}$ Yields were determined by GC using biphenyl as internal standard.

Table S4. Variation of the reaction molarity.

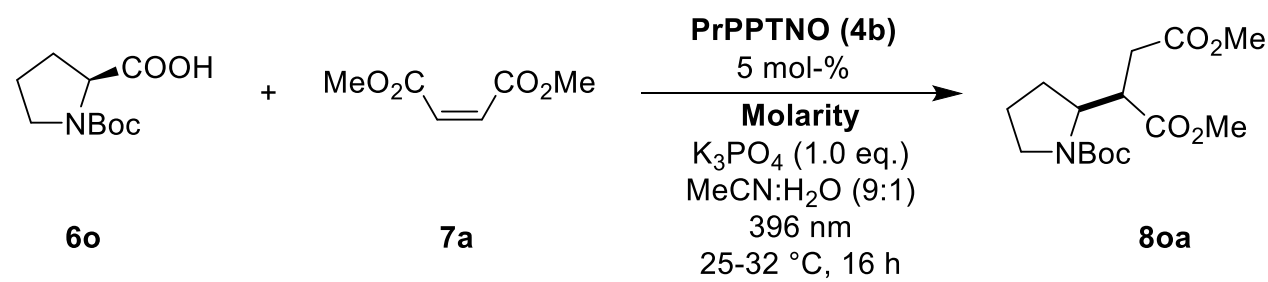

\begin{tabular}{ccc}
\hline Entry & Molarity & Yield $^{[a]}$ \\
\hline 1 & $0.05 \mathrm{M}$ & $83 \%$ \\
2 & $0.15 \mathrm{M}$ & $69 \%$ \\
3 & $0.20 \mathrm{M}$ & $50 \%$ \\
\hline
\end{tabular}

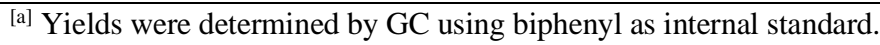


Table S5. Base screening.

\begin{tabular}{|c|c|c|c|}
\hline$\underbrace{C O O}_{60}$ & $\mathrm{CO}_{2} \mathrm{Me}$ & $\begin{array}{c}\text { PrPPTNO (4b) } \\
5 \text { mol- } \% \\
\text { Base }(1.0 \text { eq.) } \\
\text { MeCN: } \mathrm{H}_{2} \mathrm{O}(9: 1) \\
\mathrm{hv}=396 \mathrm{~nm} \\
25-32{ }^{\circ} \mathrm{C}, 16 \mathrm{~h}\end{array}$ & $\int_{\mathbf{N o a}}^{\mathrm{CO}_{2} \mathrm{Me}}$ \\
\hline Entry & Base & & Yield $^{[\mathrm{a}]}$ \\
\hline 1 & $\mathrm{~K}_{3} \mathrm{PO}_{4}$ & & $88 \%$ \\
\hline 2 & $\mathrm{~K}_{2} \mathrm{HPO}_{4}$ & & $64 \%$ \\
\hline 3 & $\mathrm{~K}_{2} \mathrm{CO}_{3}$ & & $72 \%$ \\
\hline 4 & $\mathrm{Cs}_{2} \mathrm{CO}_{3}$ & & $88 \%$ \\
\hline 5 & $\mathrm{Na}_{2} \mathrm{CO}_{3}$ & & $83 \%$ \\
\hline 6 & $\mathrm{NaHCO}_{3}$ & & $86 \%$ \\
\hline 8 & $\mathrm{NaOH}$ & & $71 \%$ \\
\hline 9 & CsF & & $85 \%$ \\
\hline 7 & $\mathrm{NaOAC}$ & & $63 \%$ \\
\hline 10 & $\mathrm{NEt}_{3}$ & & $28 \%$ \\
\hline 11 & DIPEA & & $34 \%$ \\
\hline 12 & Pyridine & & $35 \%$ \\
\hline
\end{tabular}


<smiles>COC(=O)CC(COC(C)=O)C1CCCN1C(=O)OC(C)(C)C</smiles>

$80 a$

${ }^{1} \mathrm{H}$ NMR

$\left(300 \mathrm{MHz}, \mathrm{CDCl}_{3}\right)$

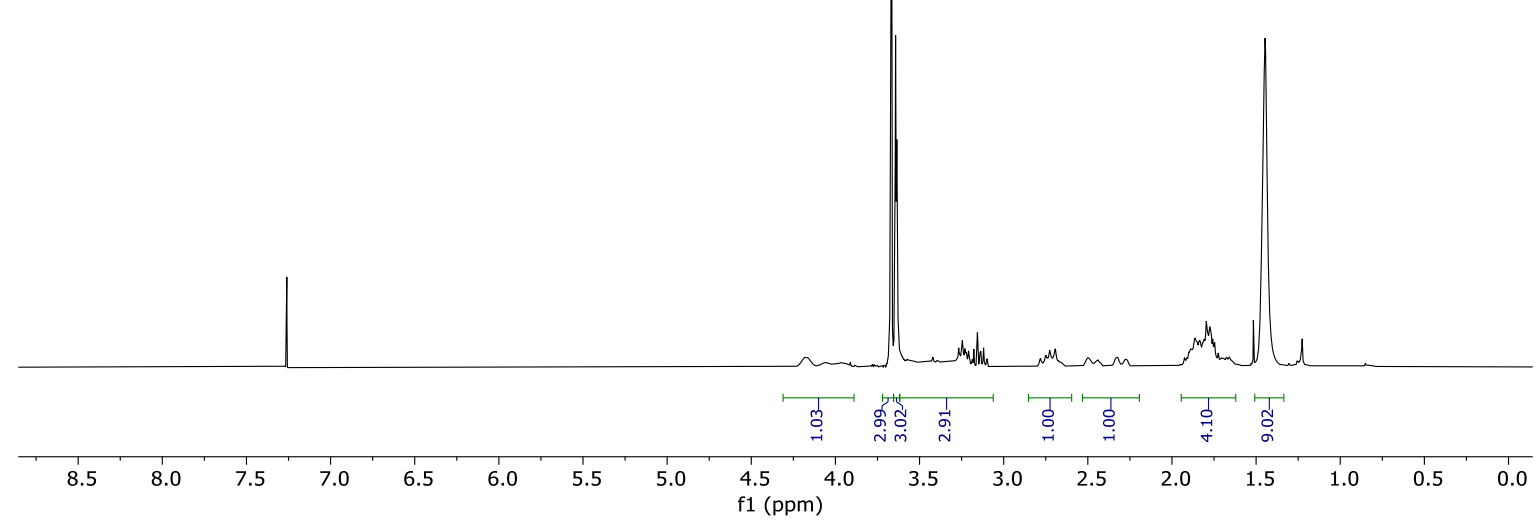

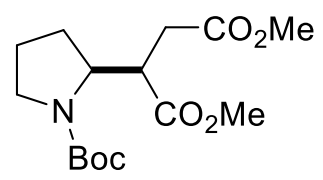

$80 a$

${ }^{13} \mathrm{C}$ NMR

$\left(75 \mathrm{MHz}^{\mathrm{CDCl}}{ }_{3}\right.$ )

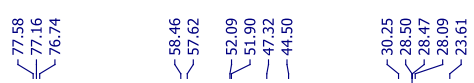

约
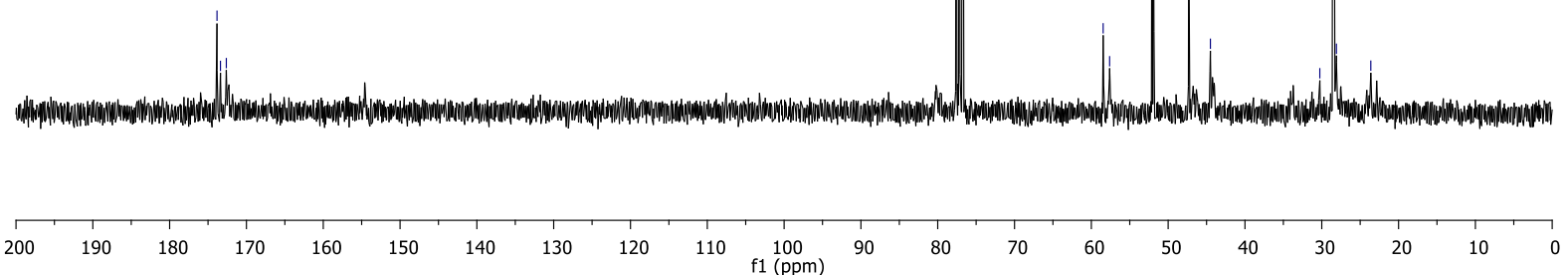

Figure S4. $1 \mathrm{H}$ - and $13 \mathrm{C}$ NMR spectra of $80 a$ 


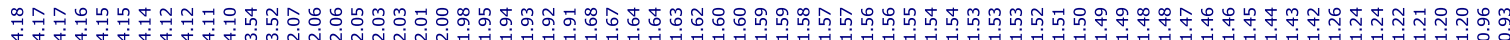<smiles>CCOC(=O)C(C)C(C)C12CC3CC(CC(C3)C1)C2</smiles>

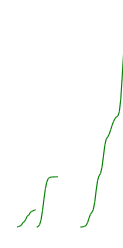

$8 \mathbf{a b}$

${ }^{1} \mathrm{H}$ NMR

$\left(300 \mathrm{MHz}, \mathrm{CDCl}_{3}\right.$ )

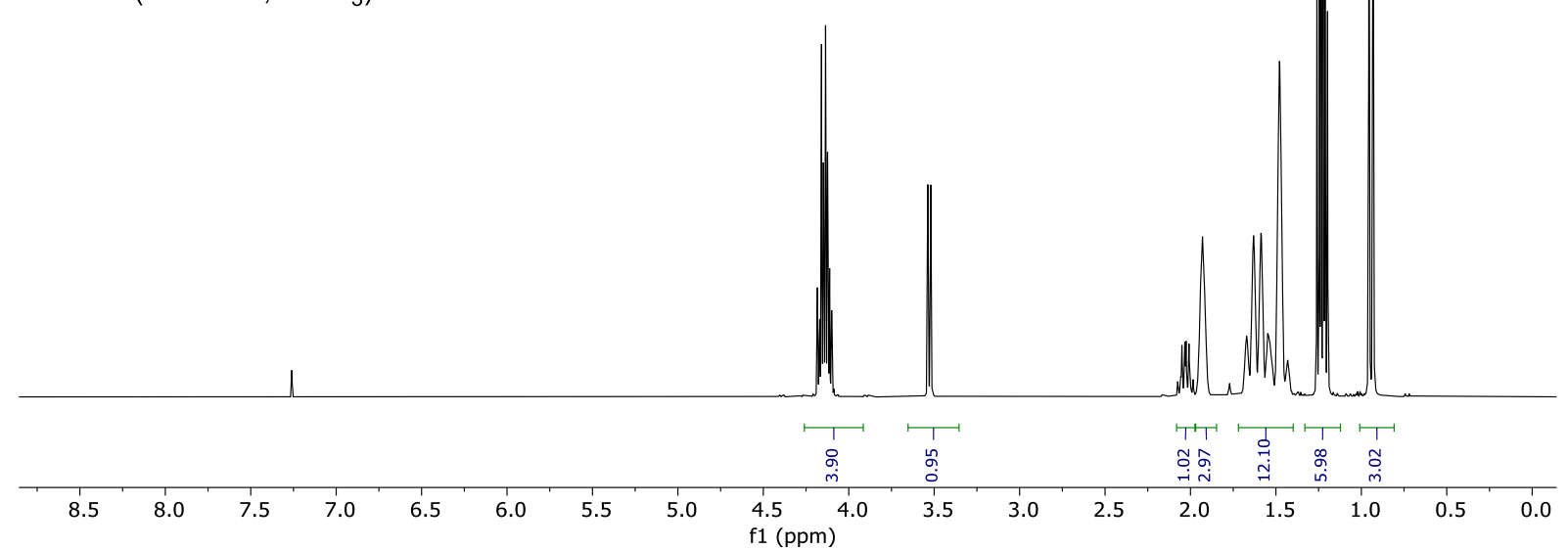

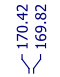<smiles>CCOC(=O)C(C)C(C)C12CC3CC(CC(C3)C1)C2</smiles>

$8 \mathrm{ab}$

${ }^{13} \mathrm{C} \mathrm{NMR}$

(75 MHz, $\mathrm{CDCl}_{3}$ )

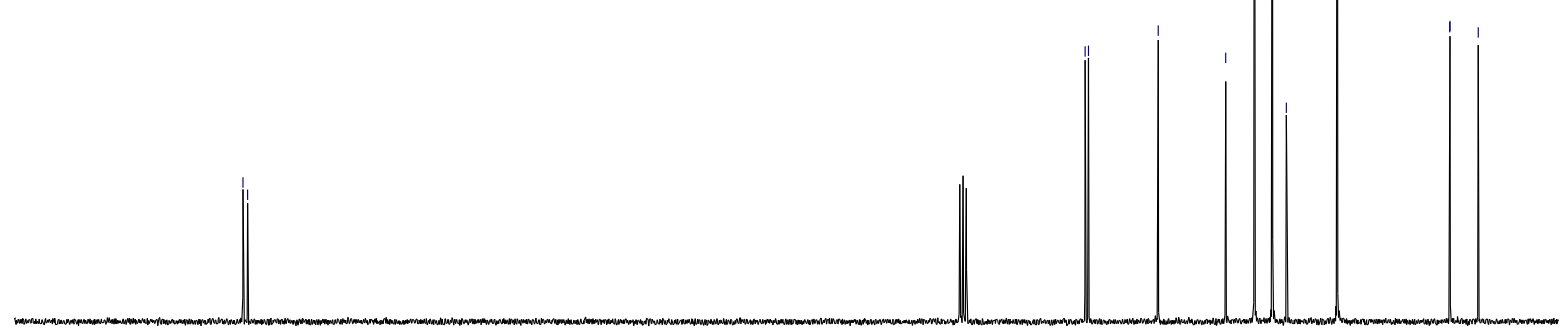

200

190

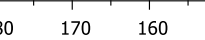

$140 \quad 1$

$10 \quad \begin{aligned} & 100 \\ & \text { f1 }\end{aligned}$

90

Figure S5. $1 \mathrm{H}$ - and $13 \mathrm{C}$ NMR spectra of $8 \mathrm{ab}$ 
<smiles>CCOC(=O)C(C)C(C)C12CCC(CC(O)(CC)C1)C2</smiles>

m

$8 \mathrm{bb}$

${ }^{1} \mathrm{H}$ NMR

$\left(300 \mathrm{MHz}, \mathrm{CDCl}_{3}\right)$

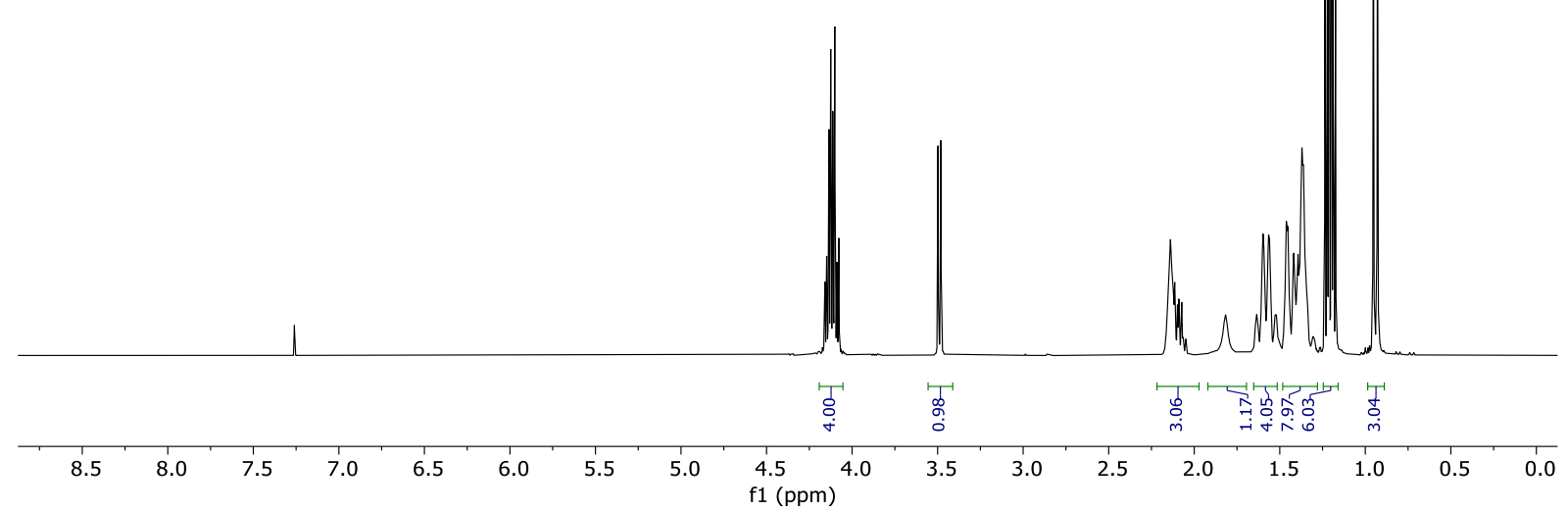<smiles>CCOC(=O)C(C)C(C)C12CC3CC(CC(O)(C3)C1)C2</smiles>

|

$8 b b$

${ }^{13} \mathrm{C}$ NMR

$\left(75 \mathrm{MHz}, \mathrm{CDCl}_{3}\right)$

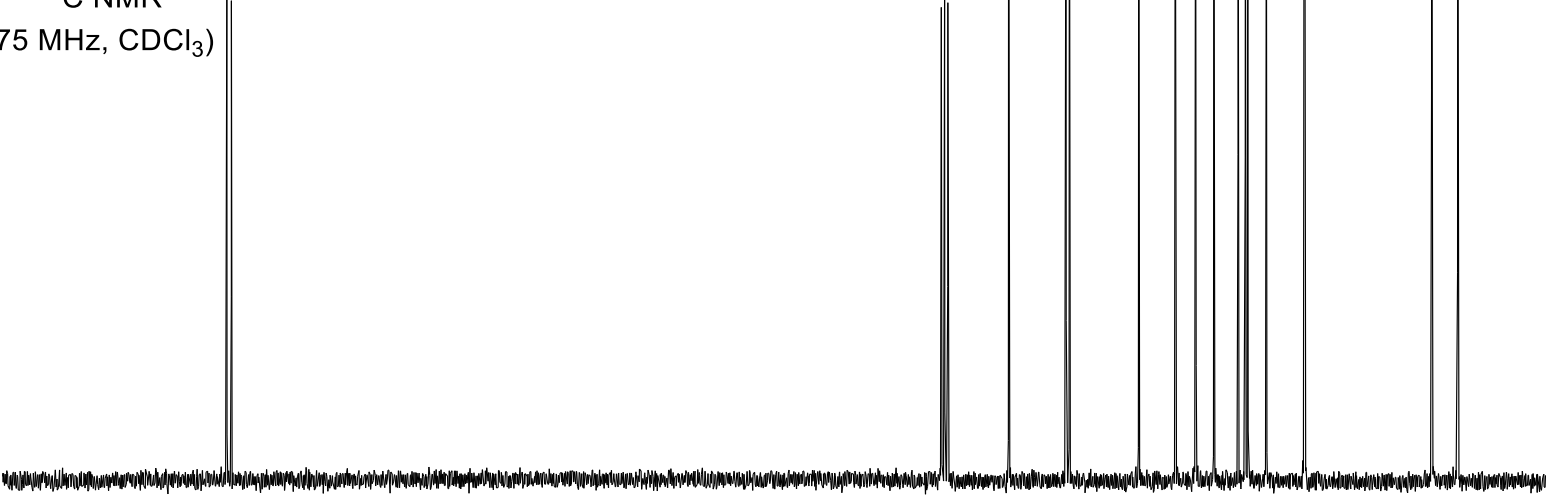

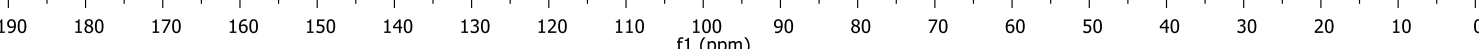

Figure $\mathbf{5 6} .1 \mathrm{H}$ - and $13 \mathrm{C}$ NMR spectra of $\mathbf{8 b b}$. 
$\mathrm{EtO}_{2} \mathrm{C}$
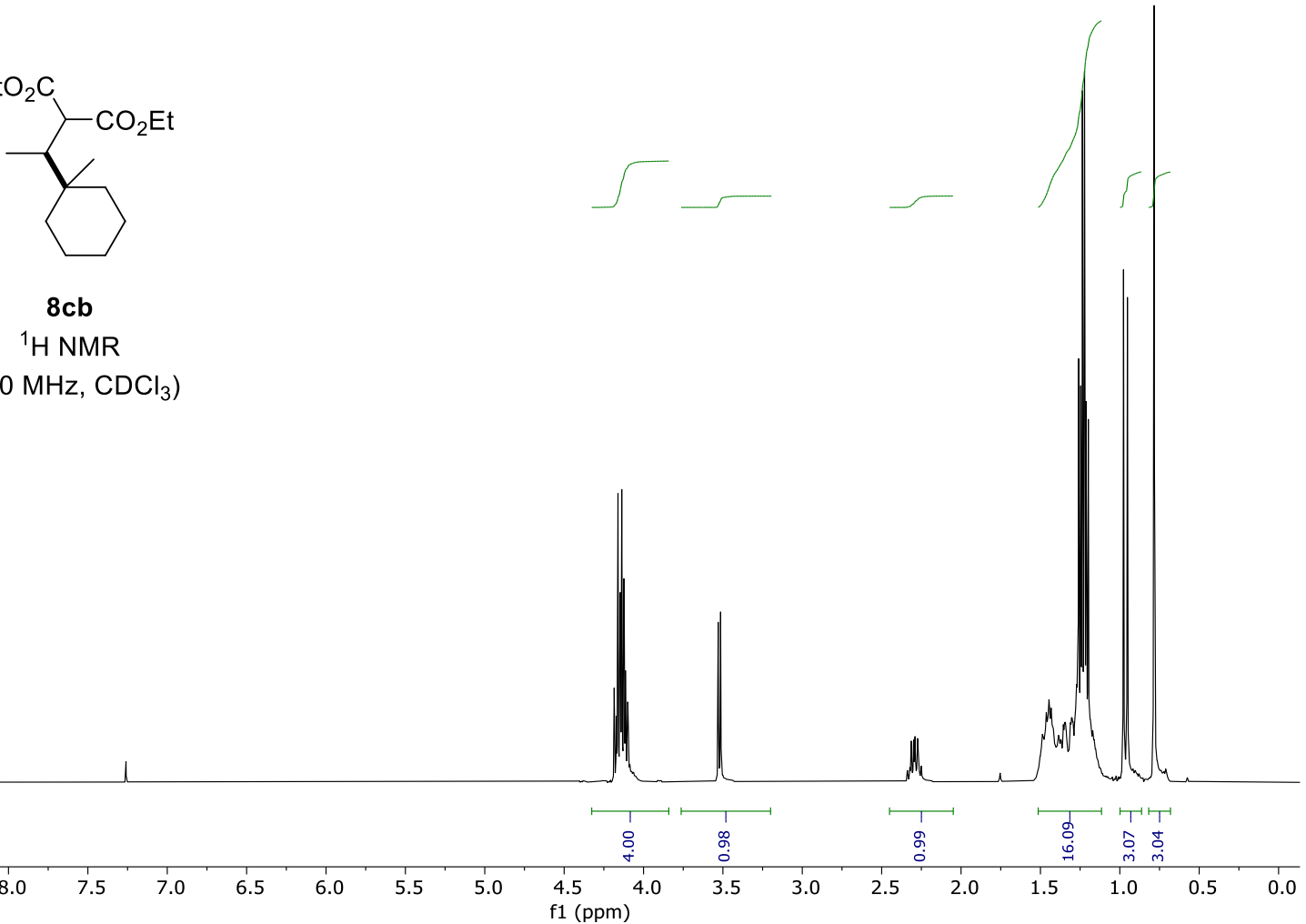

$8 \mathrm{cb}$

${ }^{1} \mathrm{H}$ NMR

$\left(300 \mathrm{MHz}, \mathrm{CDCl}_{3}\right.$ )

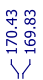

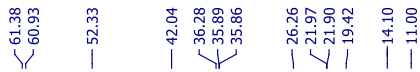

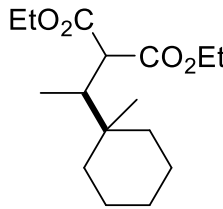

$8 \mathrm{cb}$

${ }^{13} \mathrm{C} \mathrm{NMR}$

(75 MHz, $\mathrm{CDCl}_{3}$ )

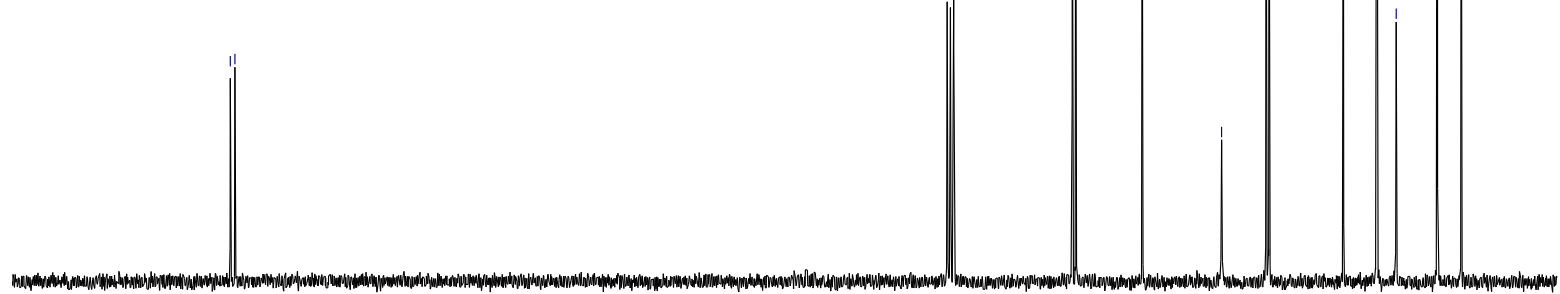

Figure S7. $1 \mathrm{H}$ - and $13 \mathrm{C}$ NMR spectra of $8 \mathrm{cb}$. 
$\mathrm{EtO}_{2} \mathrm{C}$
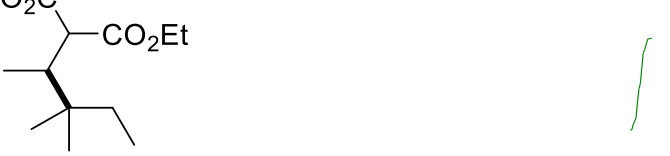

$8 d b$

${ }^{1} \mathrm{H}$ NMR

(300 MHz, $\mathrm{CDCl}_{3}$ )

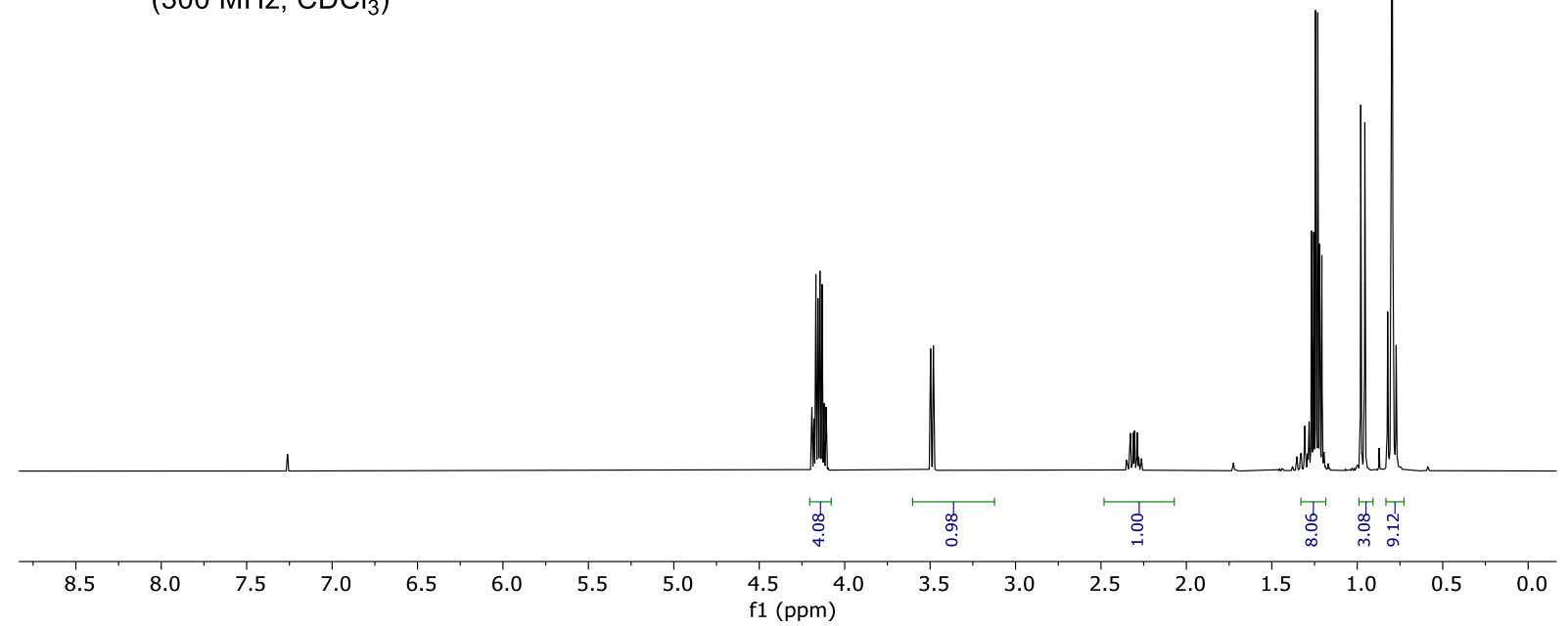

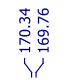

\}

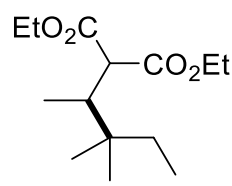

$8 \mathrm{db}$

${ }^{13} \mathrm{C}$ NMR

$\left(75 \mathrm{MHz}, \mathrm{CDCl}_{3}\right.$ )
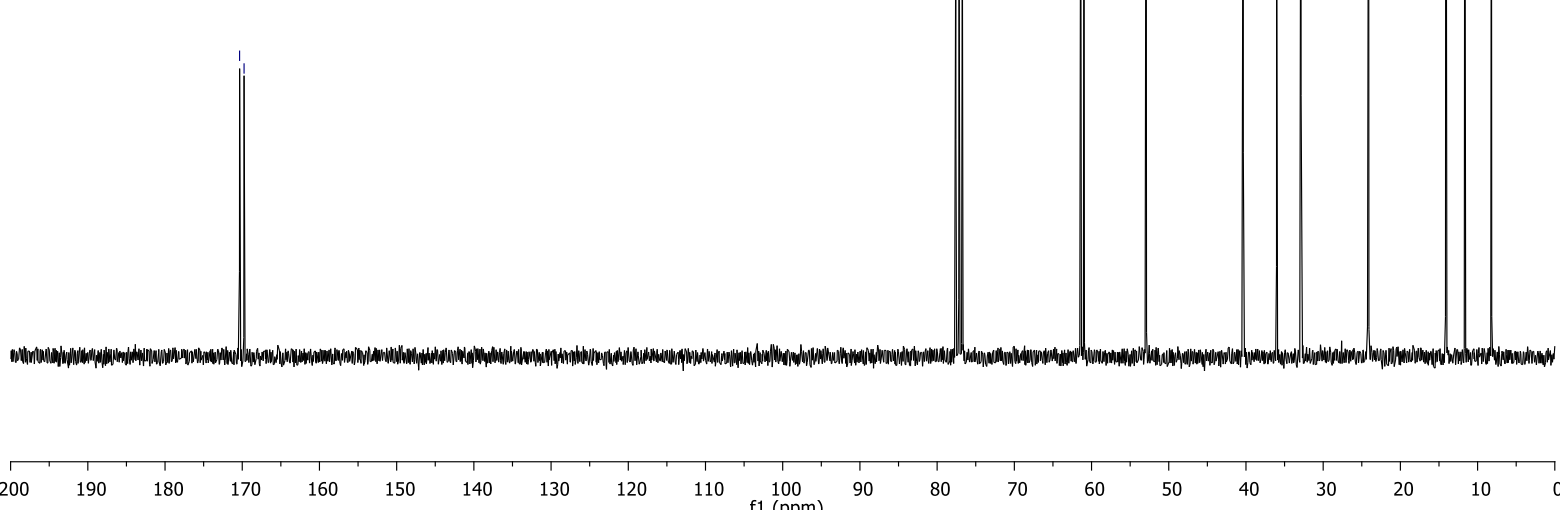

Figure $\mathbf{8 8} .1 \mathrm{H}$ - and $13 \mathrm{C}$ NMR spectra of $\mathbf{8 d b}$. 

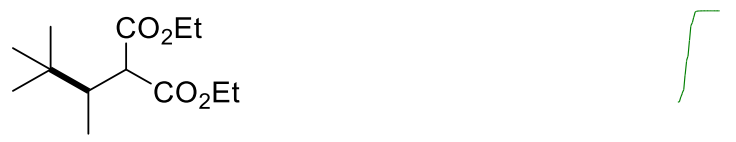

$8 \mathrm{eb}$

${ }^{1} \mathrm{H}$ NMR

(300 MHz, $\mathrm{CDCl}_{3}$ )
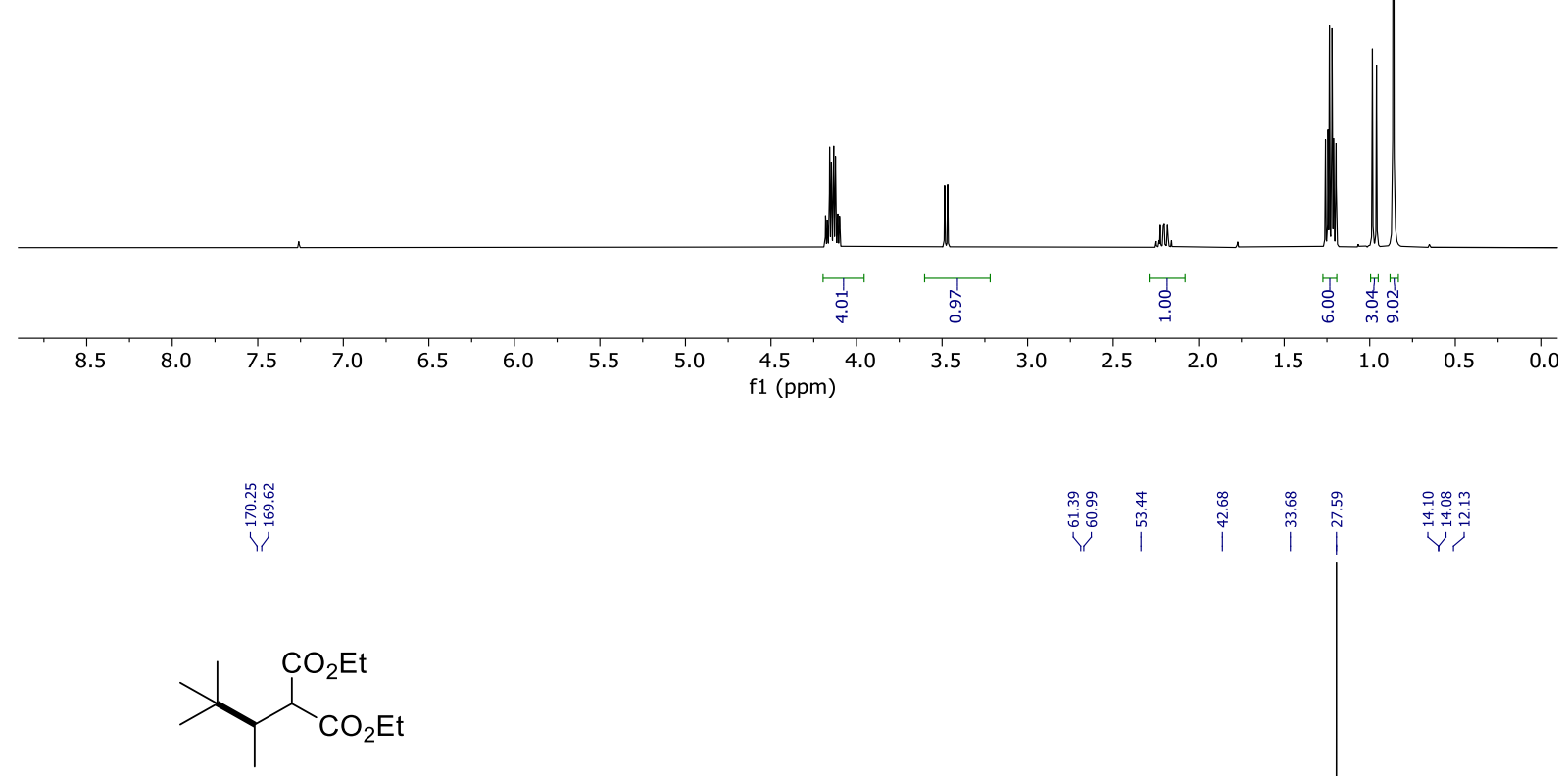

$8 \mathrm{eb}$

${ }^{13} \mathrm{C}$ NMR

(75 $\mathrm{MHz}, \mathrm{CDCl}_{3}$ )
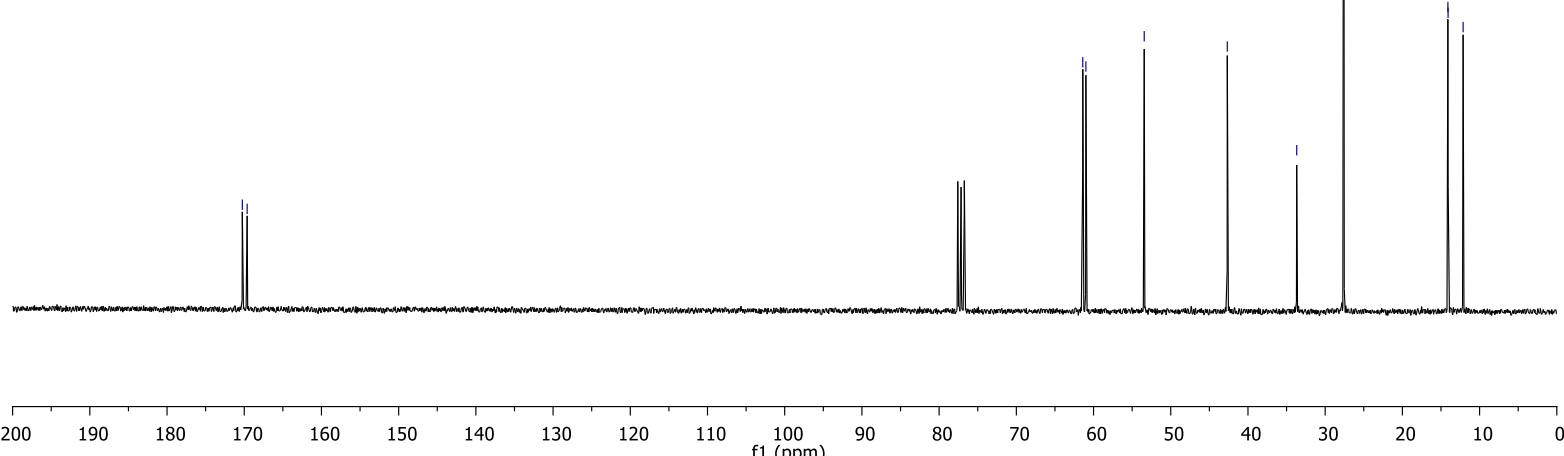

Figure S9. $1 \mathrm{H}$ - and $13 \mathrm{C}$ NMR spectra of 8eb. 


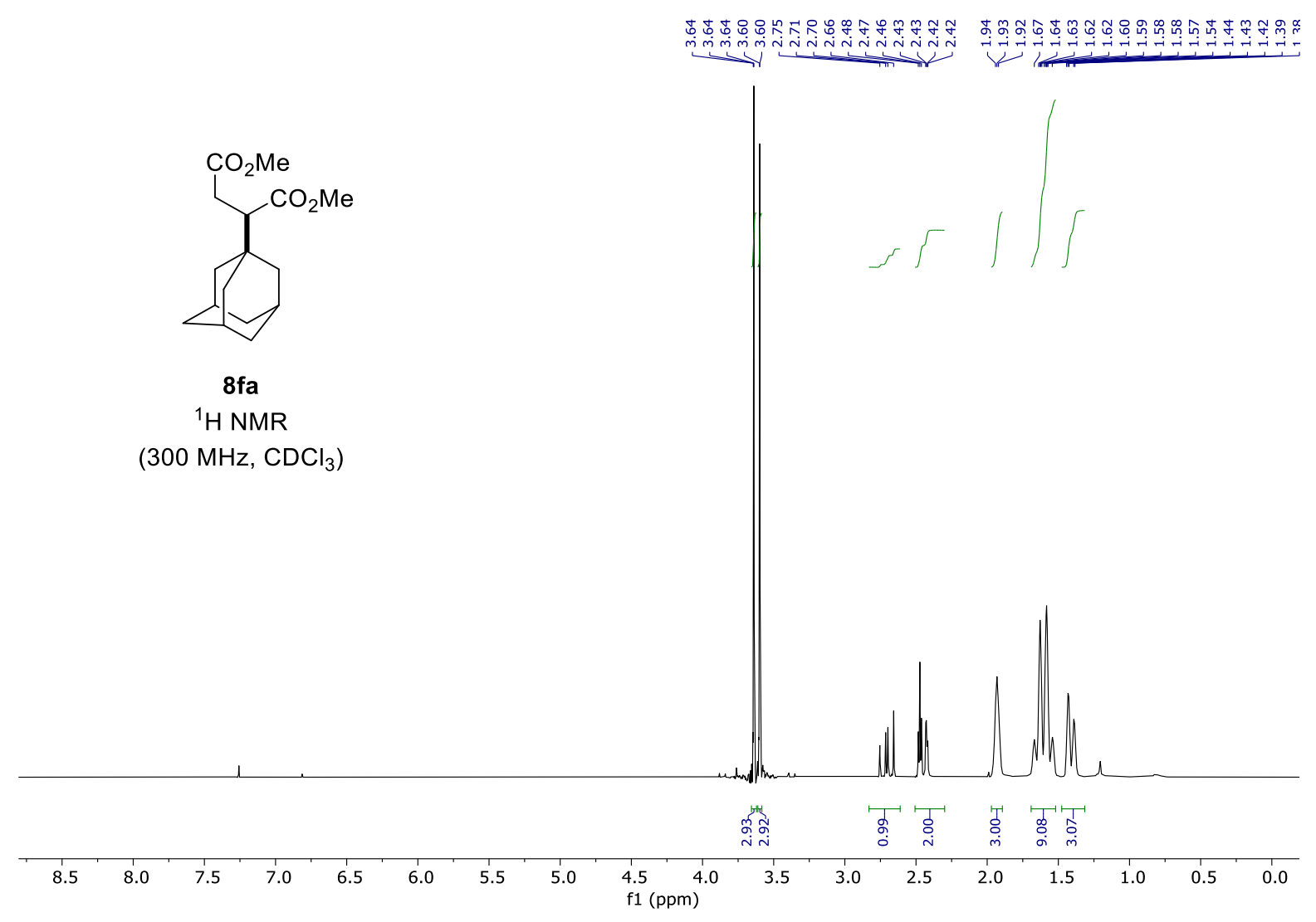

等曼
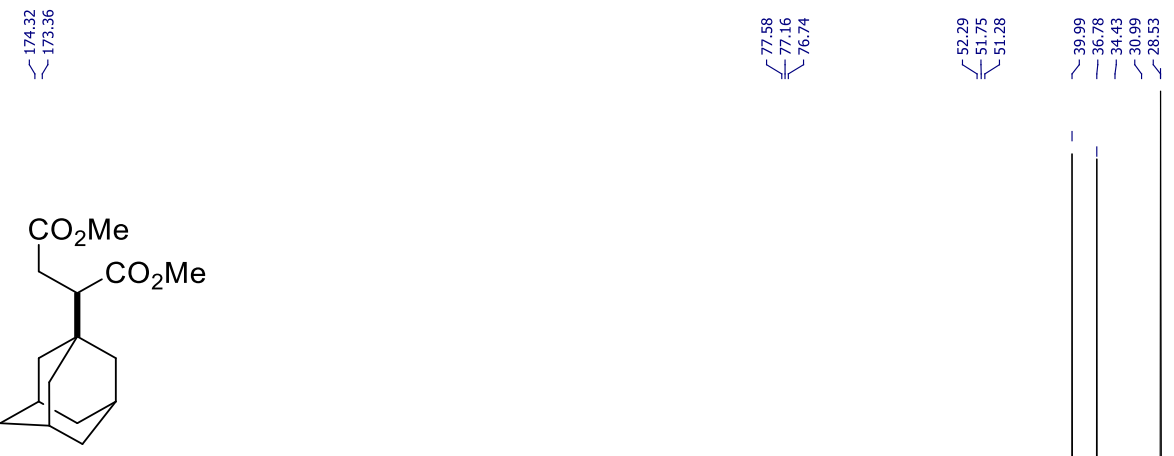

$8 \mathrm{fa}$

${ }^{13} \mathrm{C}$ NMR

(75 $\mathrm{MHz}, \mathrm{CDCl}_{3}$ )

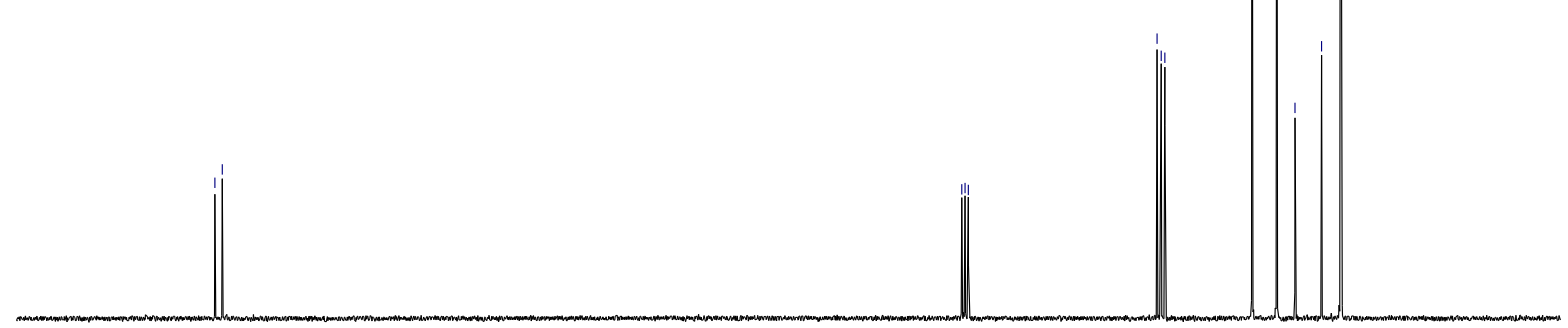

2

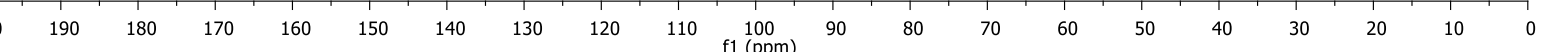

Figure $\mathbf{S 1 0} .1 \mathrm{H}$ - and $13 \mathrm{C}$ NMR spectra of $\mathbf{8 f a}$. 


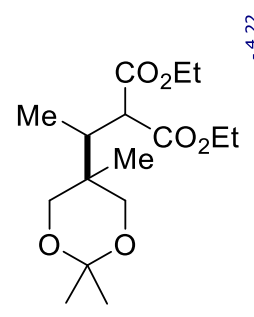

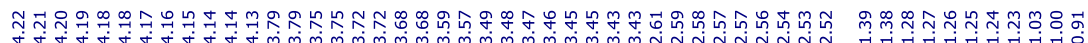

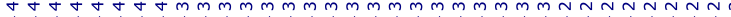

8gb

${ }^{1} \mathrm{H}$ NMR

$\left(300 \mathrm{MHz}, \mathrm{CDCl}_{3}\right)$
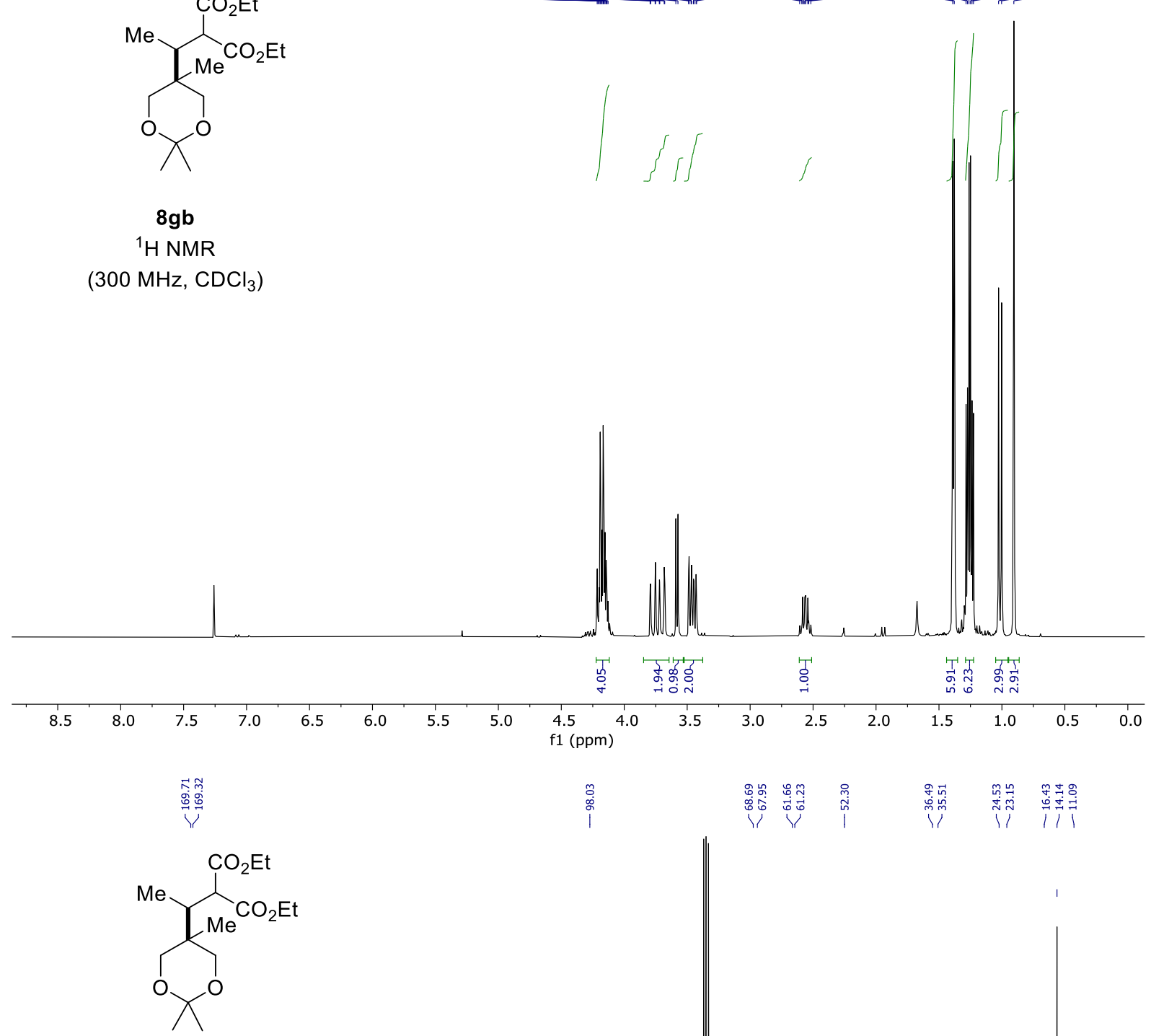

$8 \mathrm{gb}$

${ }^{13} \mathrm{C}$ NMR

$\left(75 \mathrm{MHz} \mathrm{CDCl}_{3}\right)$

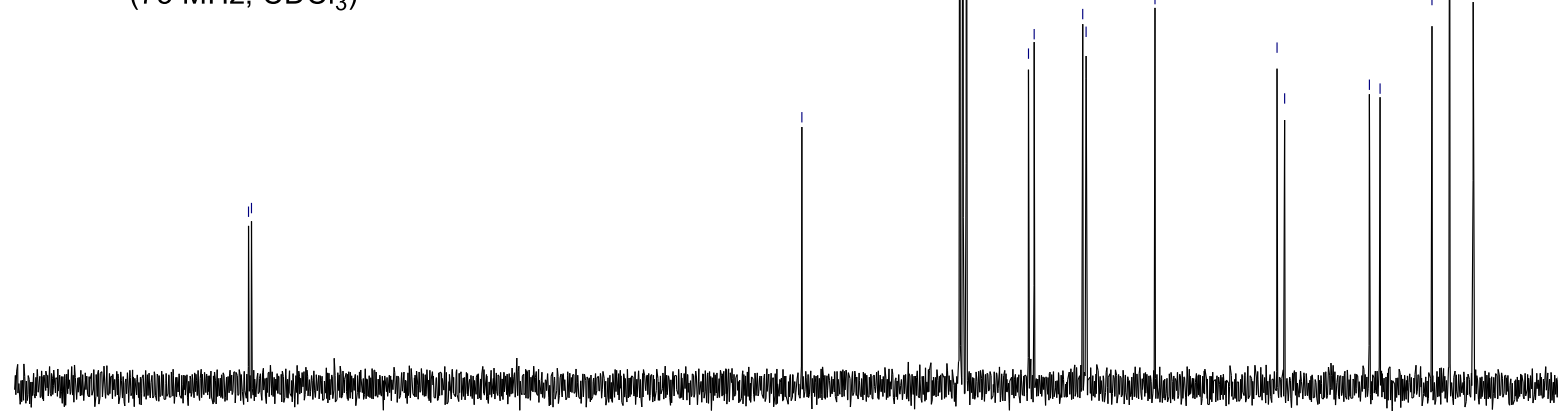

$190 \quad 180 \quad 170 \quad 160 \quad 150$

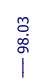

|V

\/

200
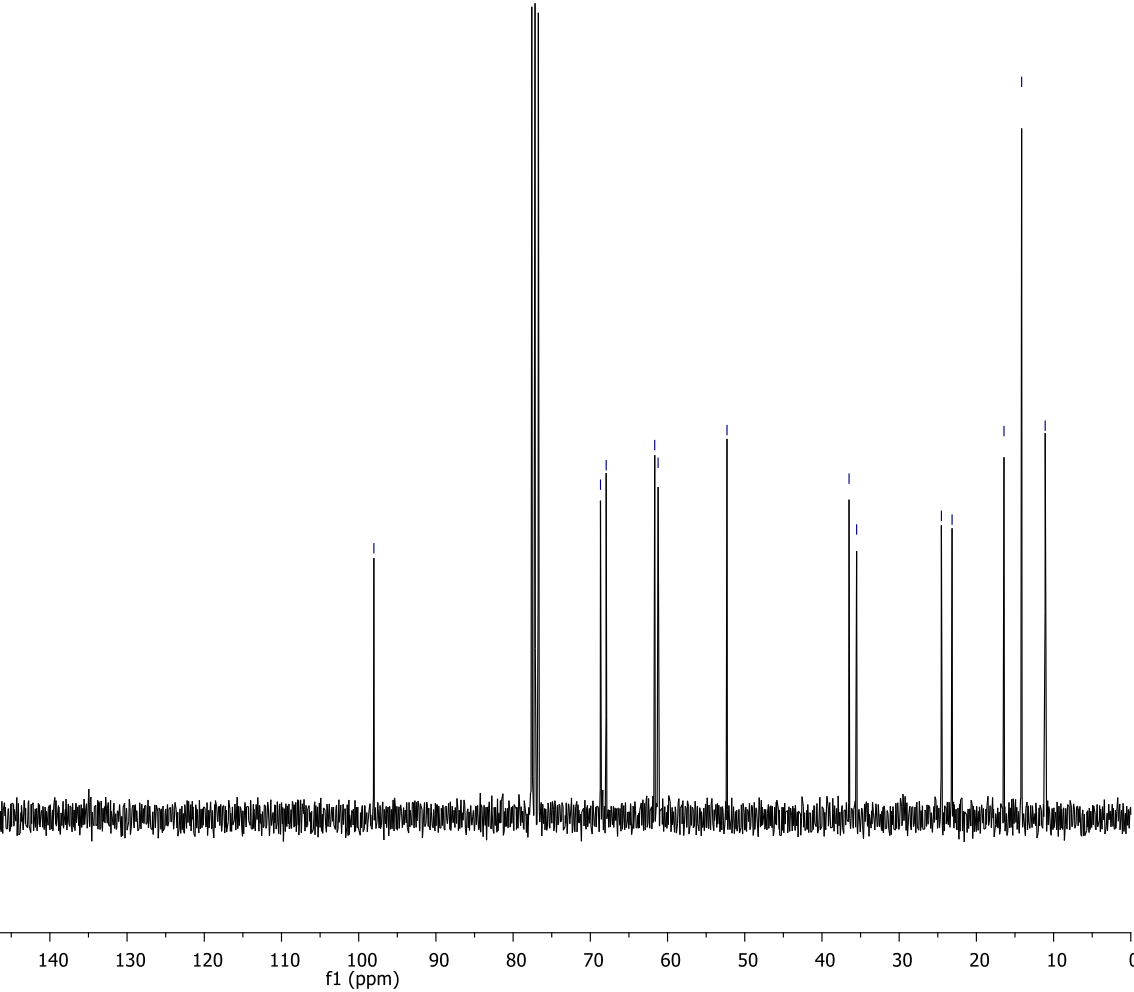

Figure $\mathbf{S 1 1 .} 1 \mathrm{H}$ - and $13 \mathrm{C}$ NMR spectra of $\mathbf{8 g b}$. 

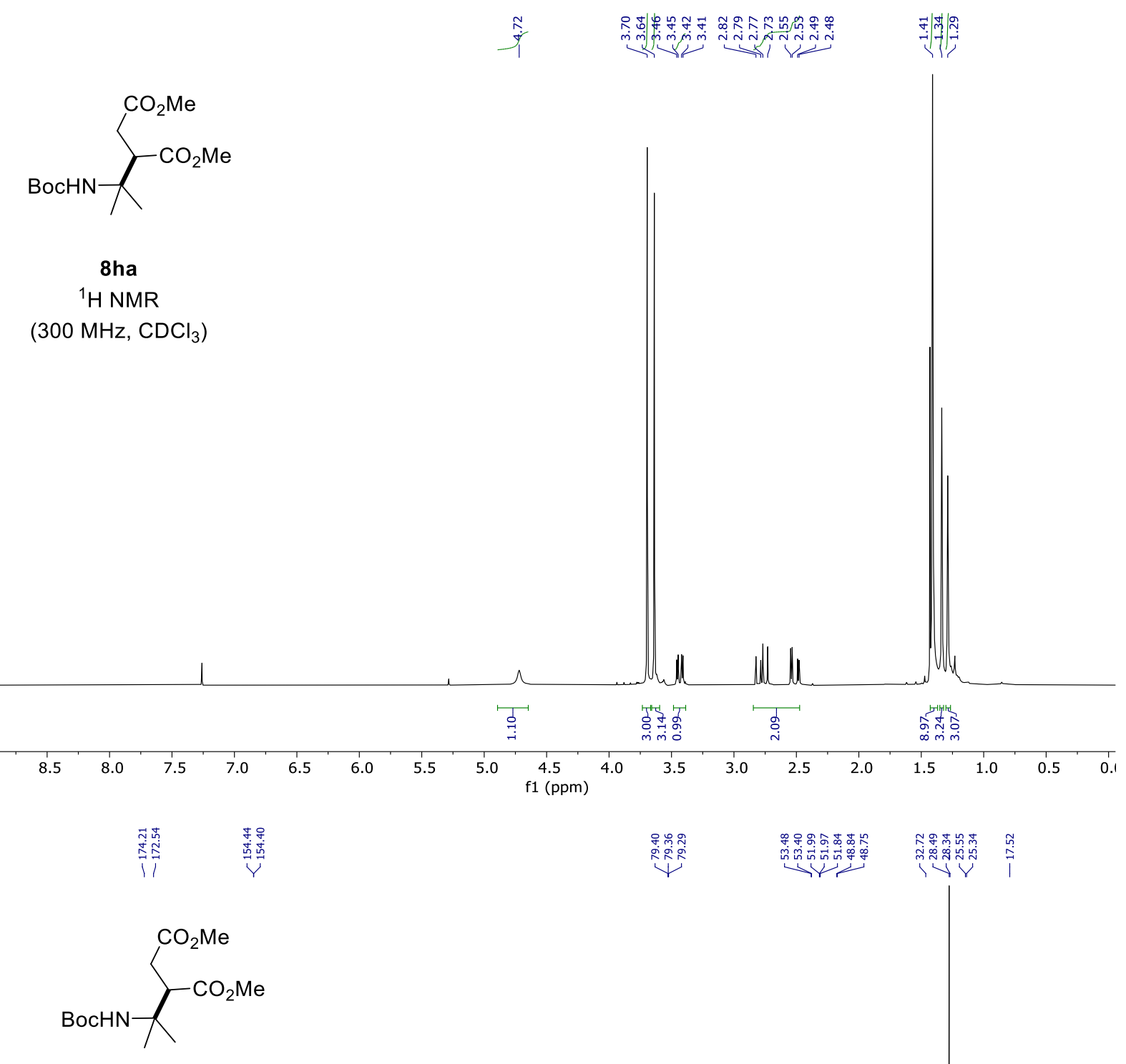

8ha

${ }^{13} \mathrm{C} \mathrm{NMR}$

(75 MHz, $\mathrm{CDCl}_{3}$ )

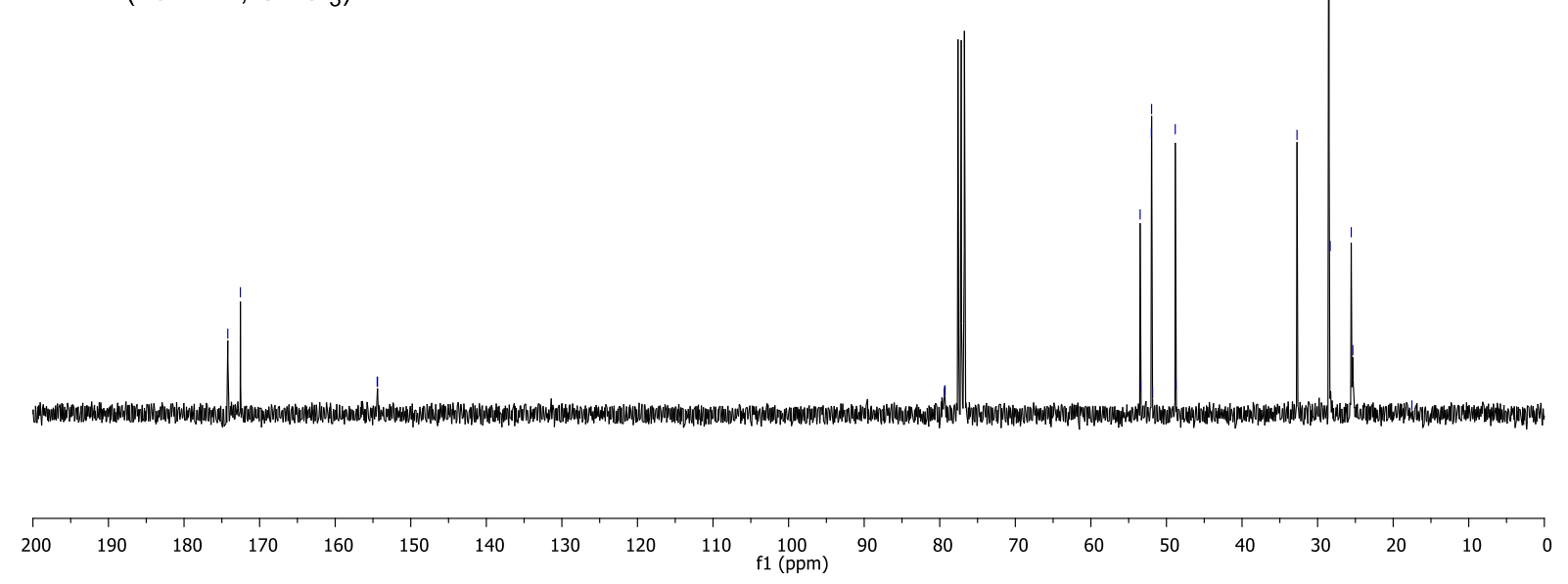

Figure S12. $1 \mathrm{H}$ - and 13C NMR spectra of 8ha. 


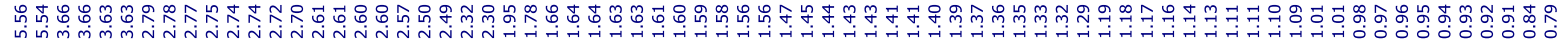

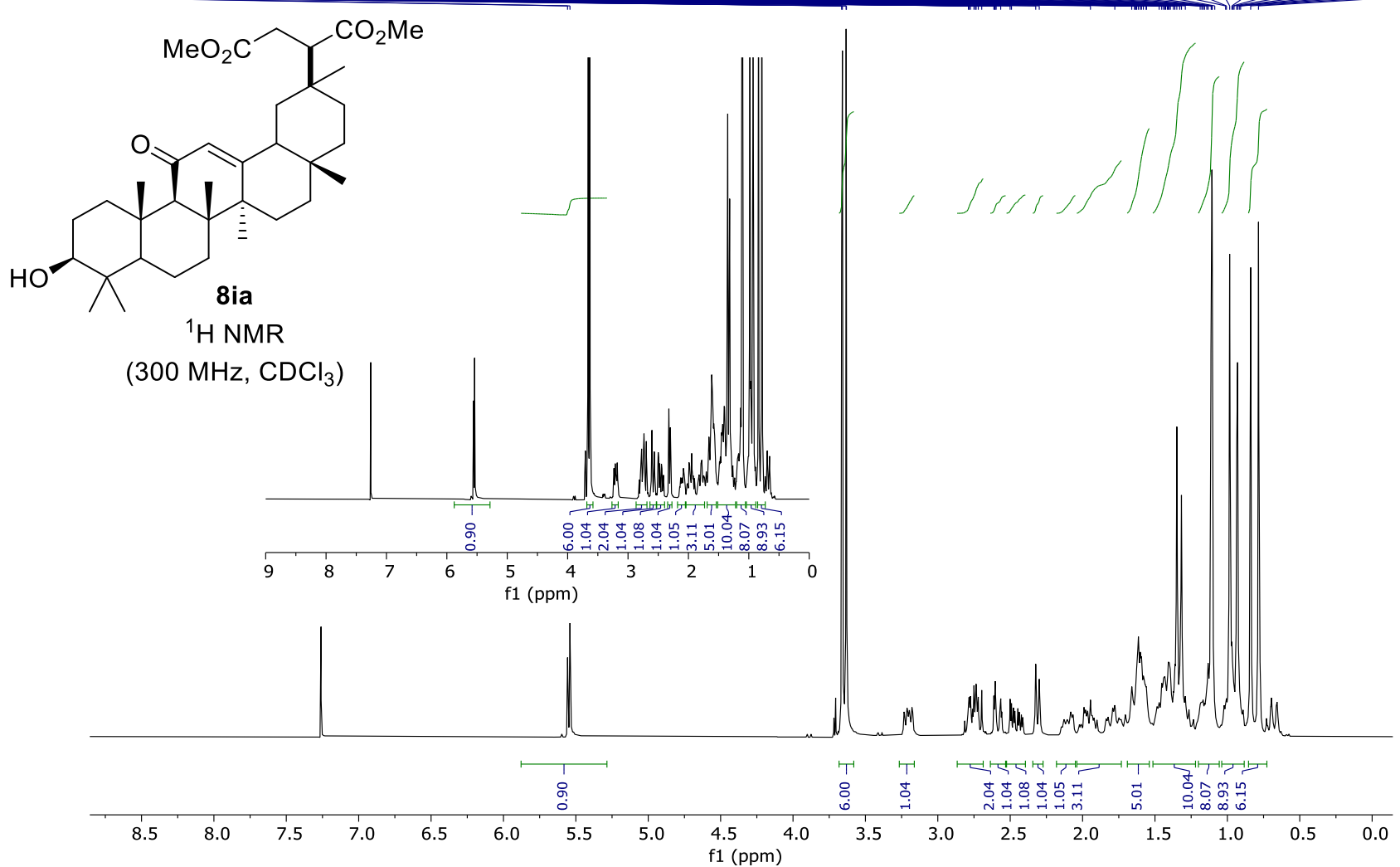

㠃

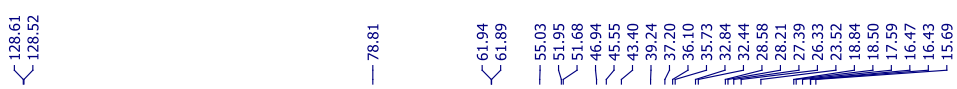

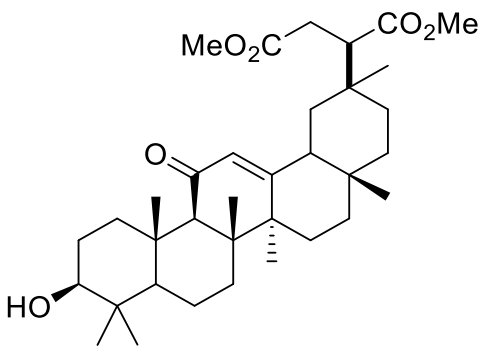

8 ia

${ }^{1} \mathrm{H}$ NMR

$\left(75 \mathrm{MHz}, \mathrm{CDCl}_{3}\right.$ )

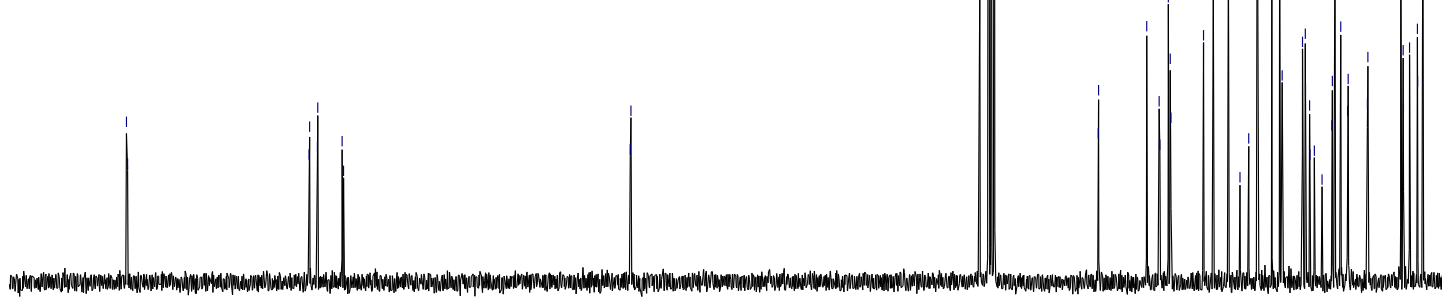

$\begin{array}{lllllllllllllllllllllllll}210 & 200 & 190 & 180 & 170 & 160 & 150 & 140 & 130 & 120 & 110 & 100 & 90 & 80 & 70 & 60 & 50 & 40 & 30 & 20 & 10 & 0\end{array}$

Figure $\mathbf{S 1 3} .1 \mathrm{H}$ - and $13 \mathrm{C}$ NMR spectra of $8 \mathrm{ia}$. 

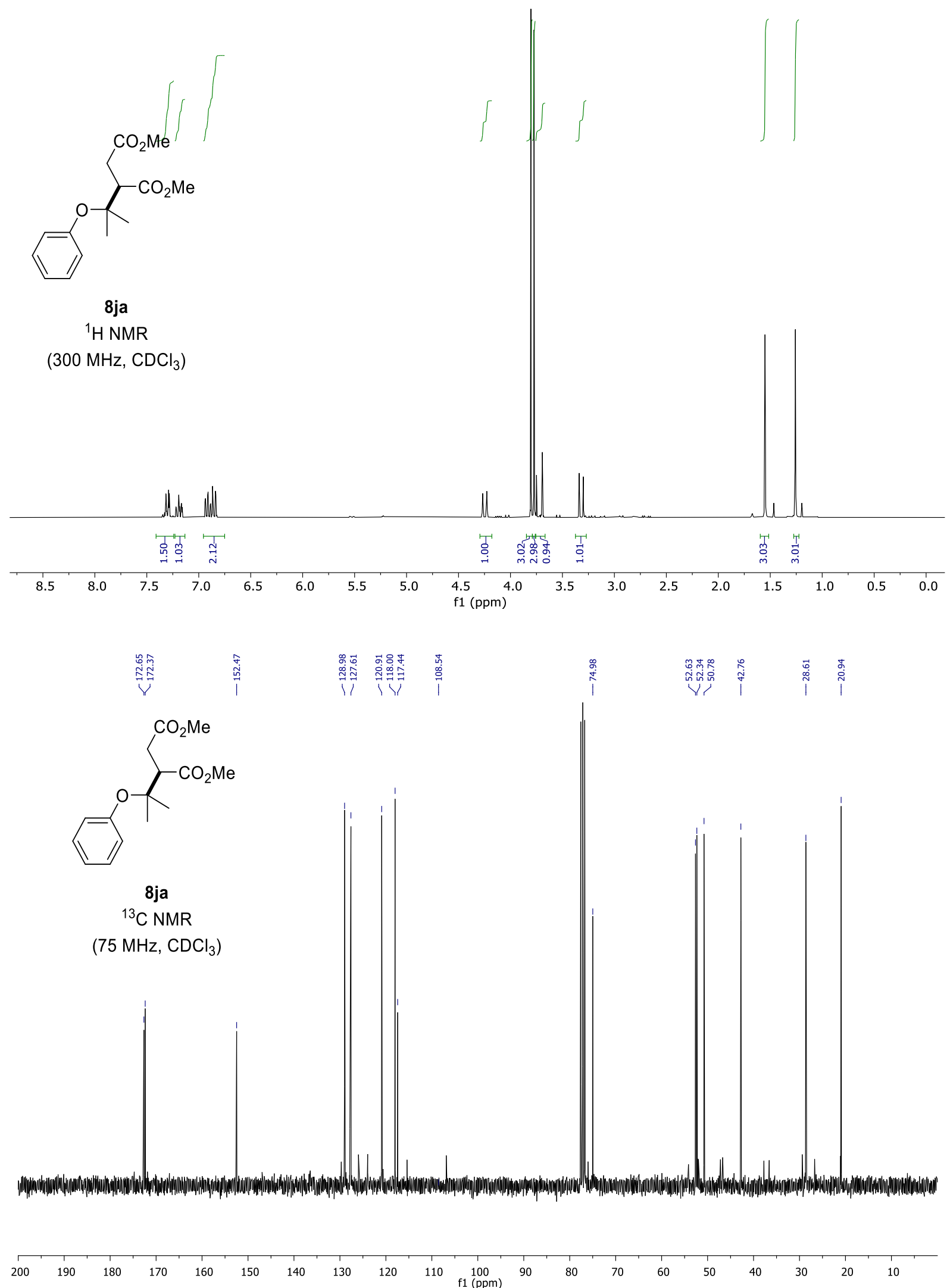

Figure S14. $1 \mathrm{H}$ - and 13C NMR spectra of $8 \mathbf{j a}$. 
<smiles>COC(=O)CC(CCCCOc1cc(C)ccc1C)C(C)(C)CC(C)=O</smiles>

$8 \mathbf{k a}$

${ }^{1} \mathrm{H}$ NMR

(300 MHz, $\mathrm{CDCl}_{3}$ )

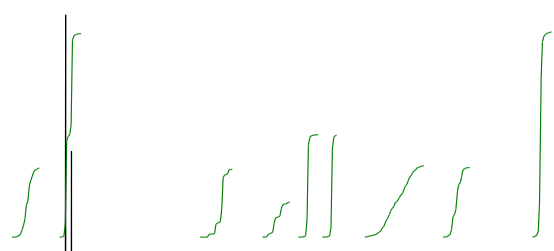

h

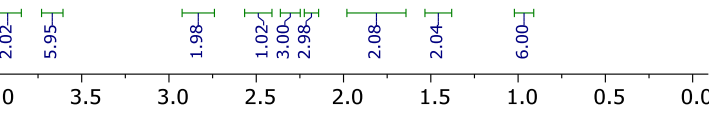

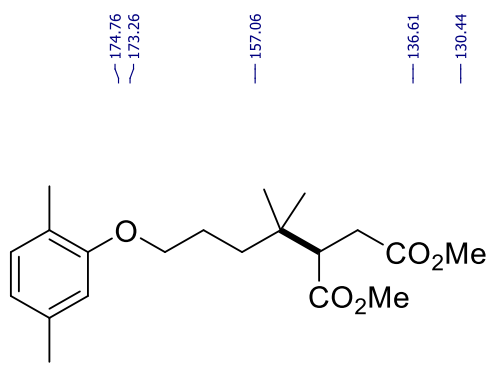

$8 \mathrm{ka}$

${ }^{13} \mathrm{C}$ NMR

$\left.\left(75 \mathrm{MHz}^{\mathrm{CDCl}}\right)_{3}\right)$

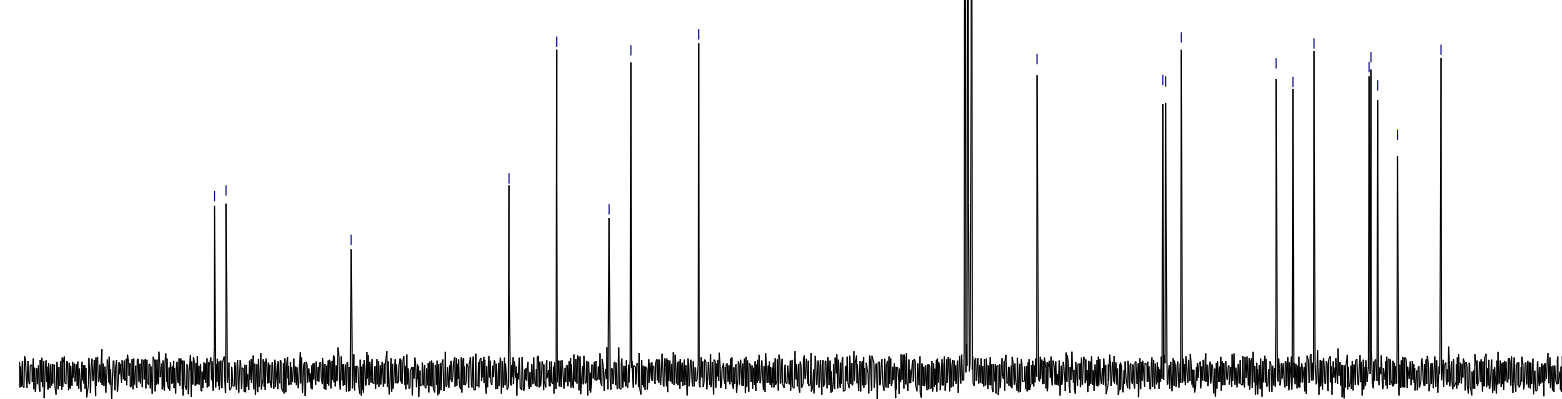

|

$\begin{array}{lllllllllll}200 & 190 & 180 & 170 & 160 & 150 & 140 & 130 & 120 & 110 & \begin{array}{c}100 \\ \mathrm{f} 1(\mathrm{ppm})\end{array}\end{array}$

Figure $\mathbf{S 1 5} .1 \mathrm{H}$ - and $13 \mathrm{C}$ NMR spectra of $\mathbf{8 k a}$ 


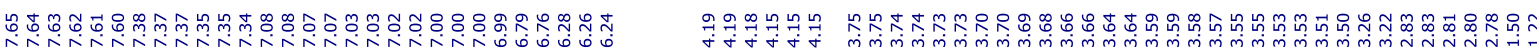
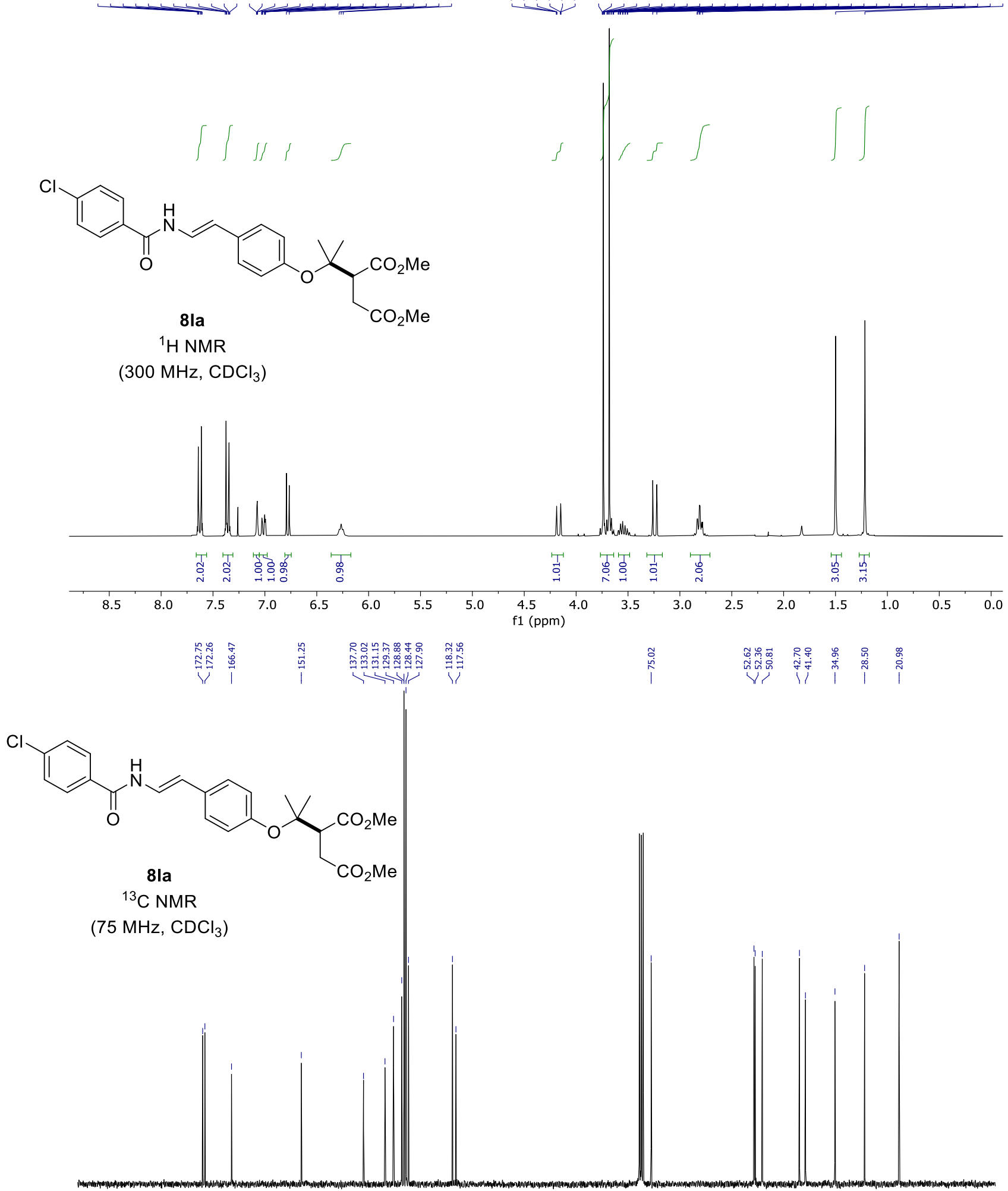

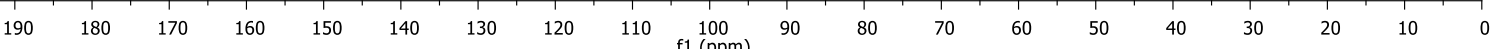

Figure S16. $1 \mathrm{H}$ - and 13C NMR spectra of 8la. 

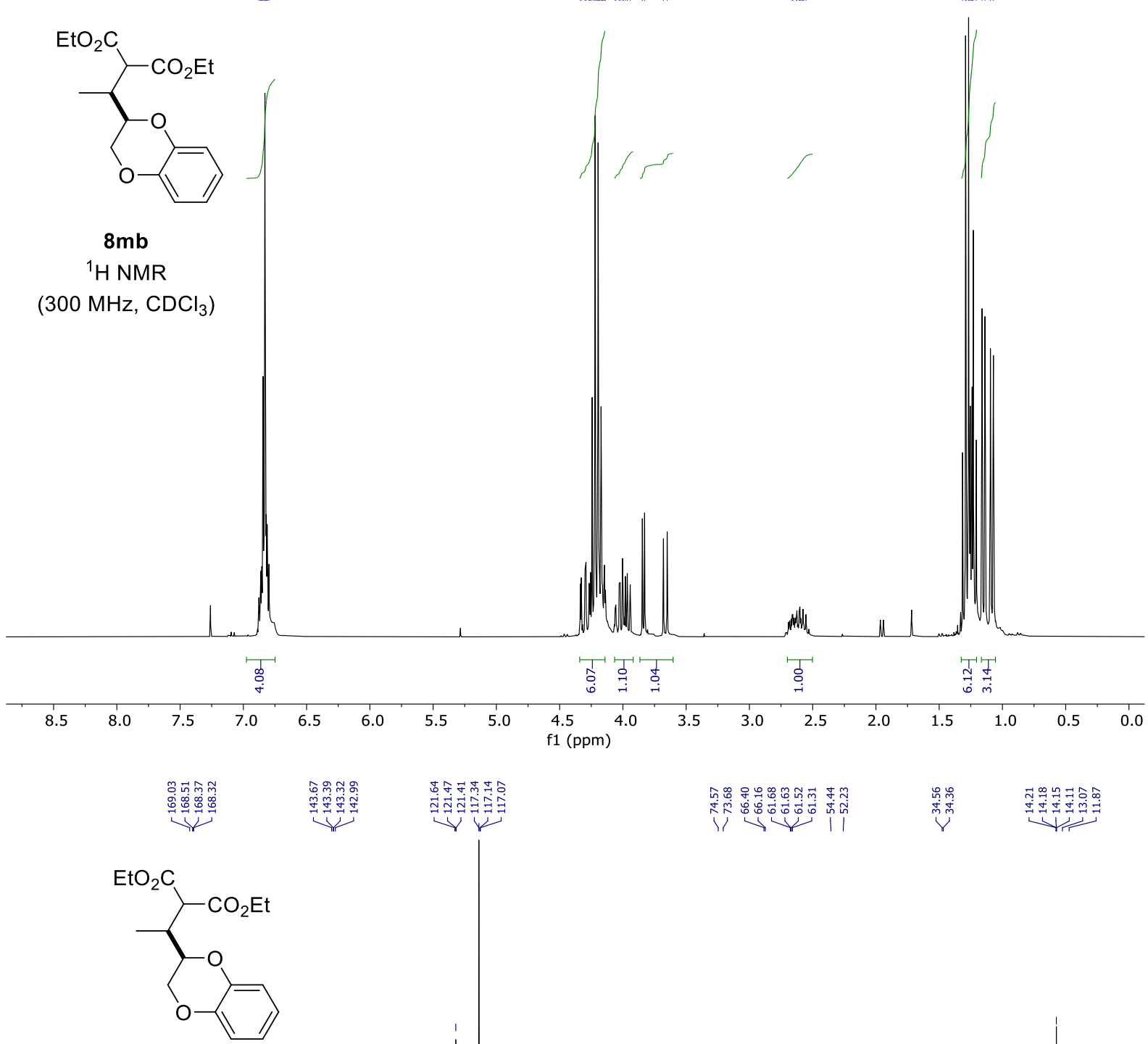

$8 \mathrm{mb}$

${ }^{13} \mathrm{C}$ NMR

(75 MHz, $\mathrm{CDCl}_{3}$ )

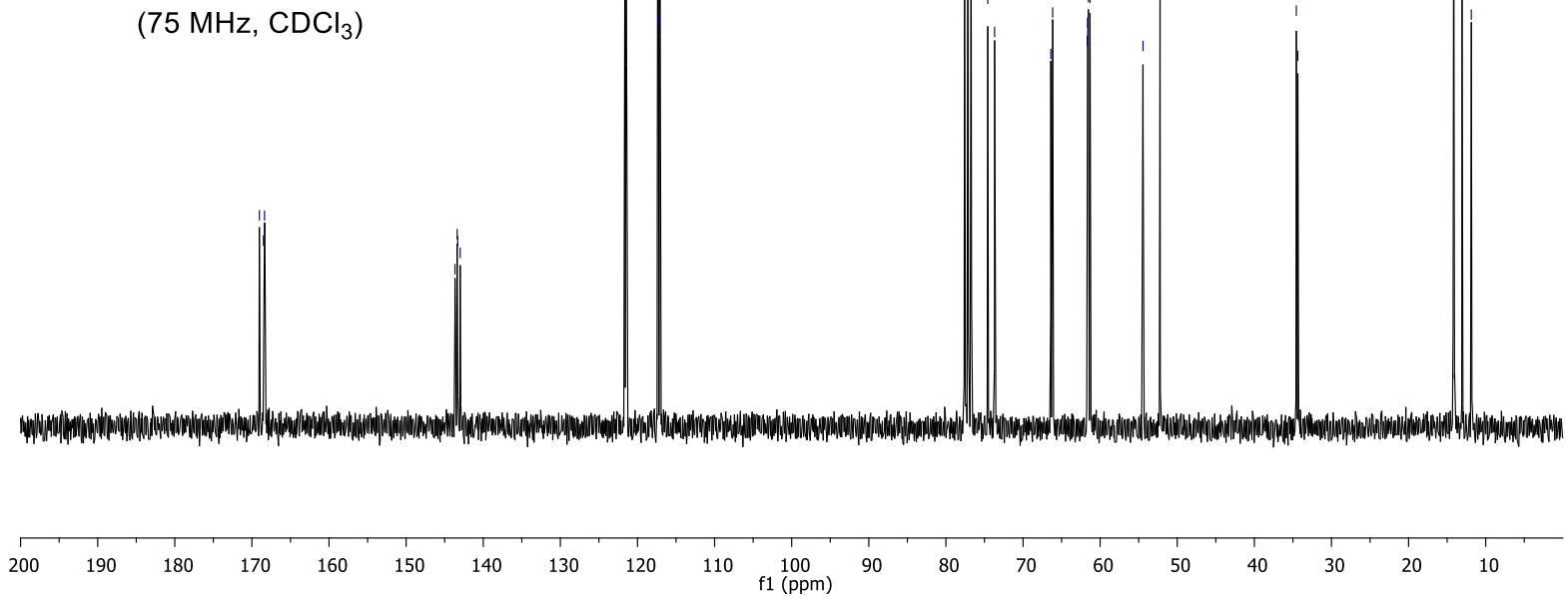

Figure S17. $1 \mathrm{H}$ - and 13C NMR spectra of $8 \mathrm{mb}$. 

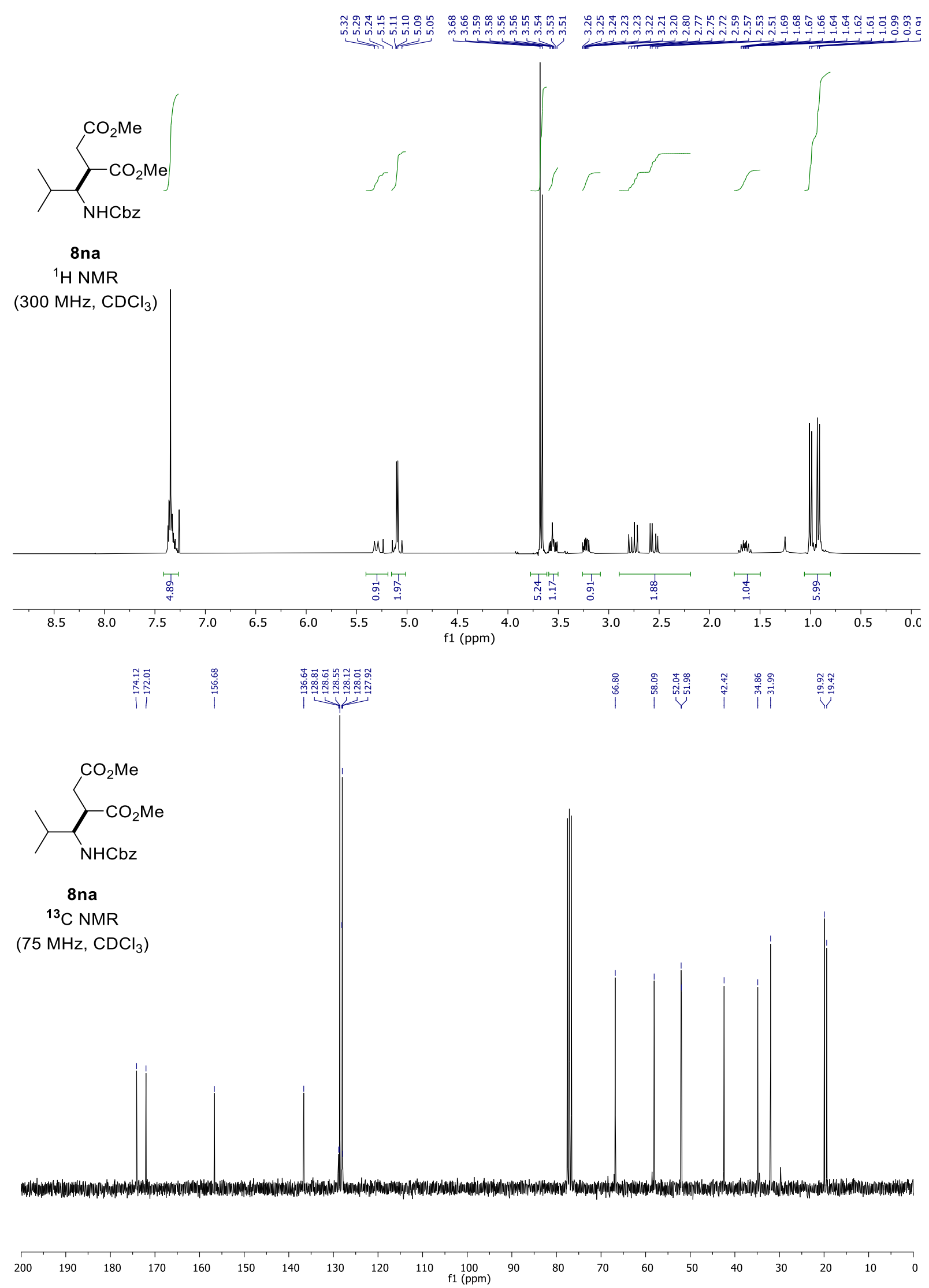

Figure S18. $1 \mathrm{H}$ - and 13C NMR spectra of 8na. 

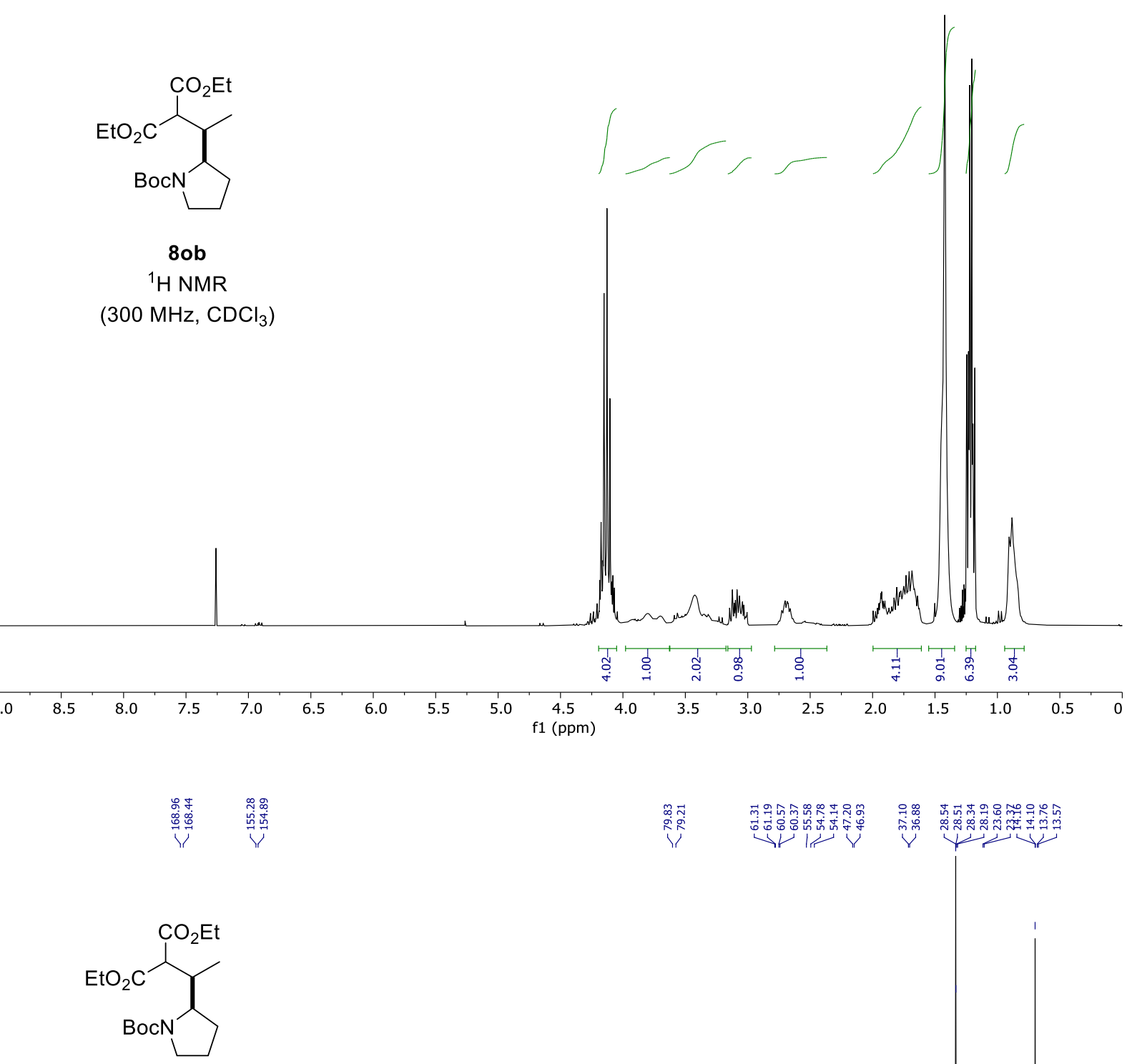

$80 b$

${ }^{13} \mathrm{C}$ NMR

$\left(75 \mathrm{MHz}, \mathrm{CDCl}_{3}\right)$

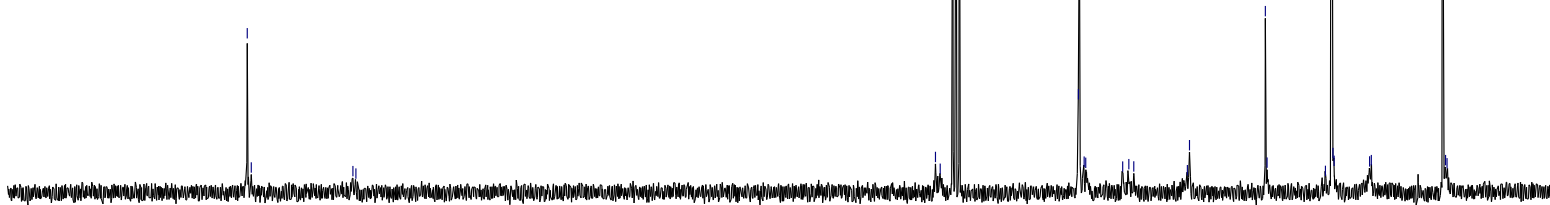

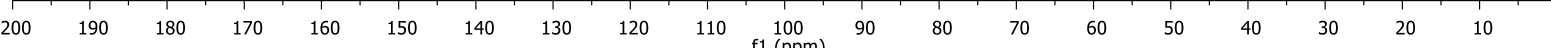

Figure S19. $1 \mathrm{H}$ - and 13C NMR spectra of 8ob. 


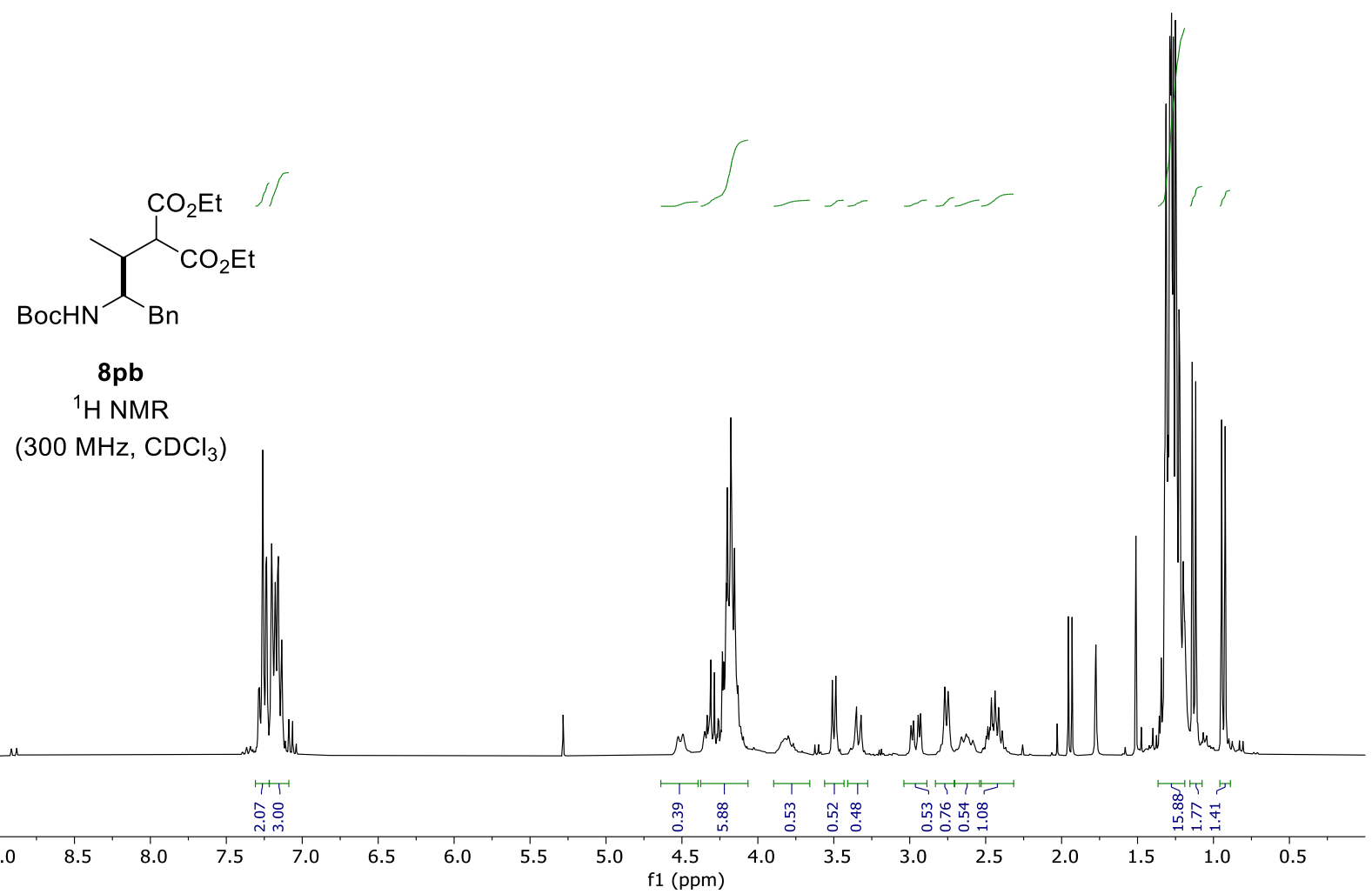<smiles>CCOC(=O)C(C)C(Cc1ccccc1)NC(C)(C)C</smiles>

$8 \mathrm{pb}$

${ }^{13} \mathrm{C}$ NMR

$\left(75 \mathrm{MHz}, \mathrm{CDCl}_{3}\right)$

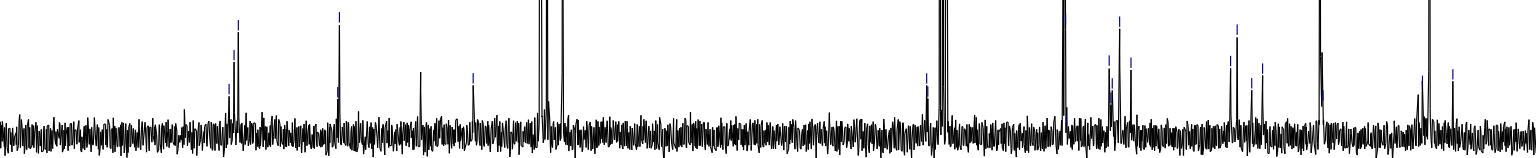

Figure S20. $1 \mathrm{H}$ - and 13C NMR spectra of $\mathbf{8 p b}$. 

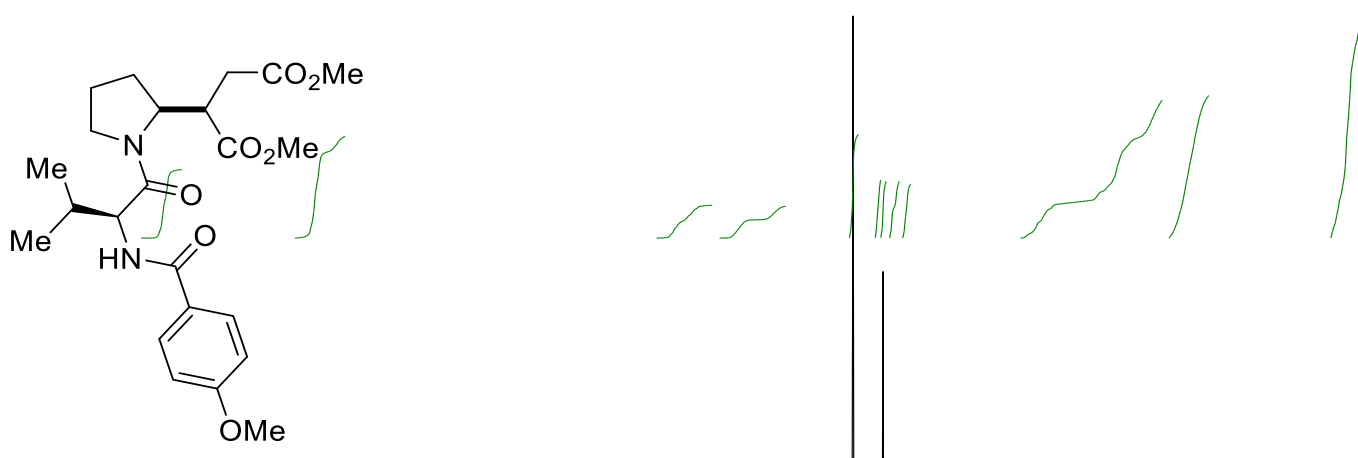

$8 q a$

${ }^{1} \mathrm{H}$ NMR

(300 $\left.\mathrm{MHz}, \mathrm{CDCl}_{3}\right)$

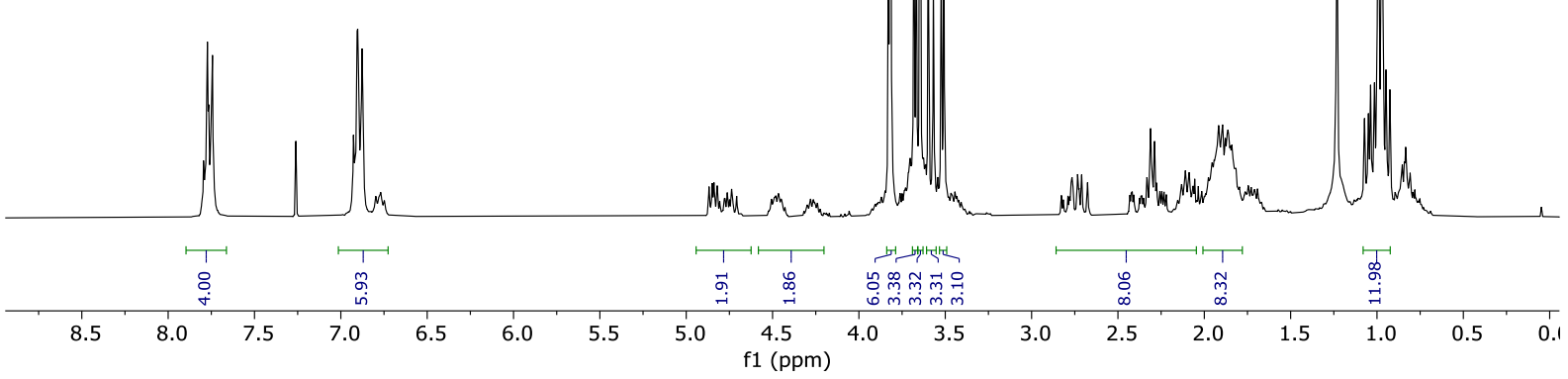

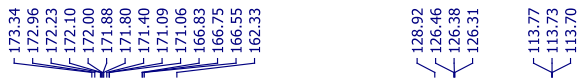

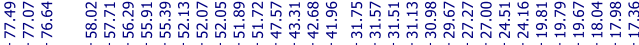

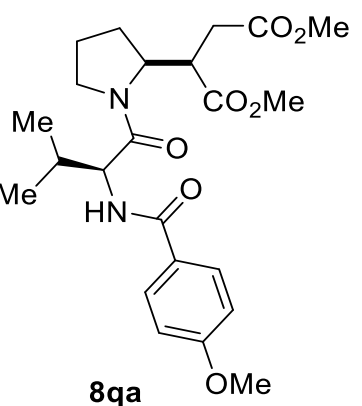

${ }^{13} \mathrm{C}$ NMR

$\left.\left(75 \mathrm{MHz}^{\mathrm{CDCl}}\right)_{3}\right)$

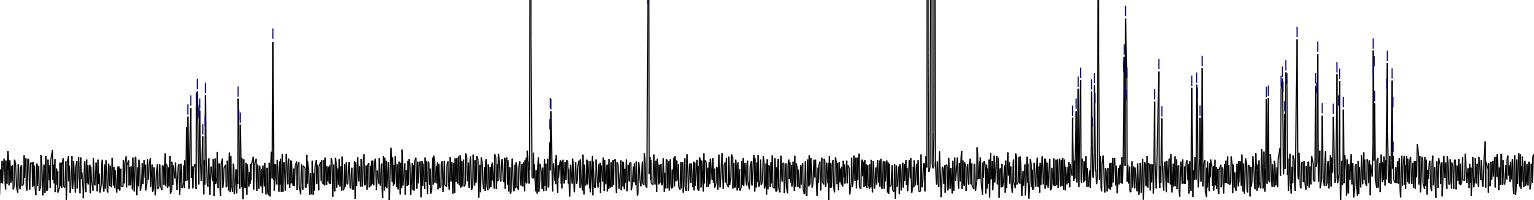

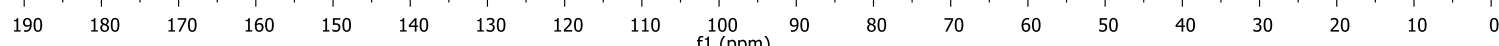

Figure $\mathbf{2 1} .1 \mathrm{H}$ - and 13C NMR spectra of 8qa. 
<smiles>CCOC(=O)C(C)C(C)C1CCCO1</smiles>

$8 \mathrm{rb}$

${ }^{1} \mathrm{H} \mathrm{NMR}$

(300 MHz, $\mathrm{CDCl}_{3}$ )
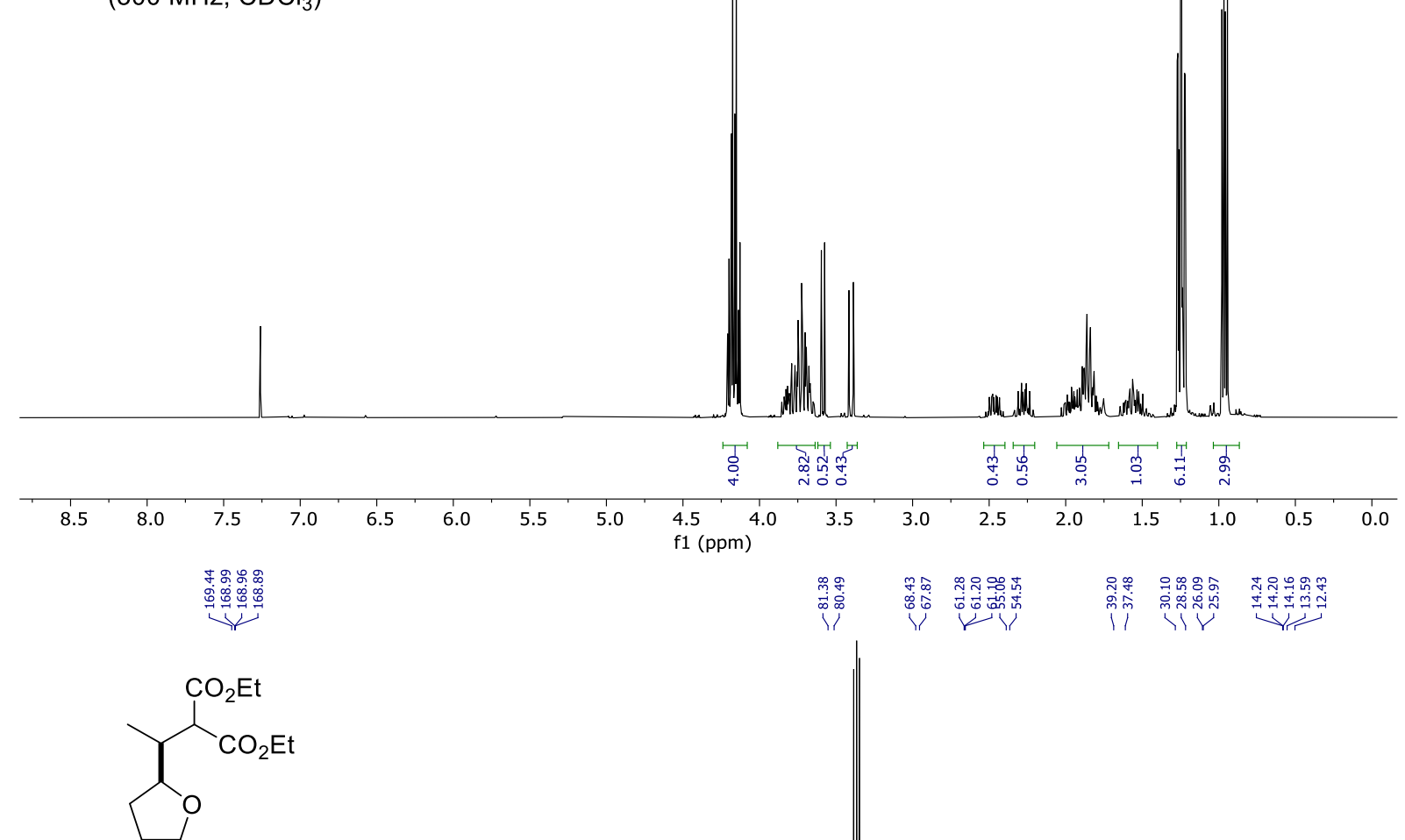

$8 \mathrm{rb}$

${ }^{13} \mathrm{C}$ NMR

(75 $\mathrm{MHz}, \mathrm{CDCl}_{3}$ )

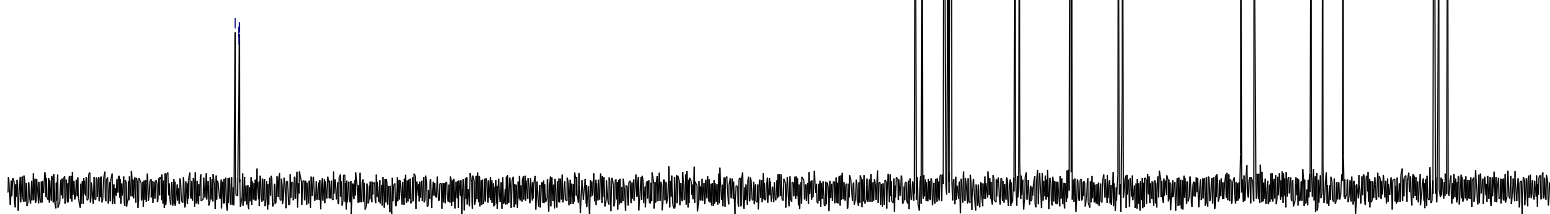

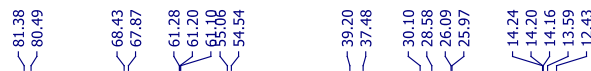

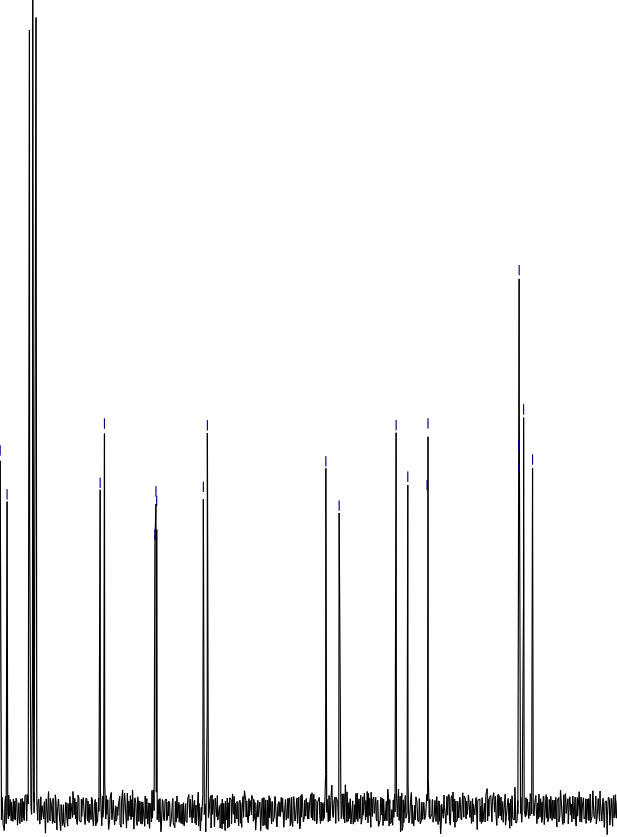

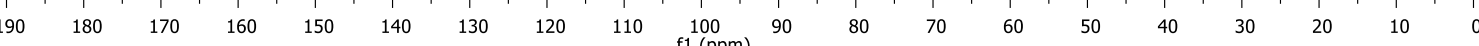

Figure S22. 1H- and 13C NMR spectra of 8rb. 
<smiles>CCOC(=O)C(C)C(C)C1CCCO1</smiles>

8sa (a)

${ }^{1} \mathrm{H}$ NMR

$\left(300 \mathrm{MHz}, \mathrm{CDCl}_{3}\right)$

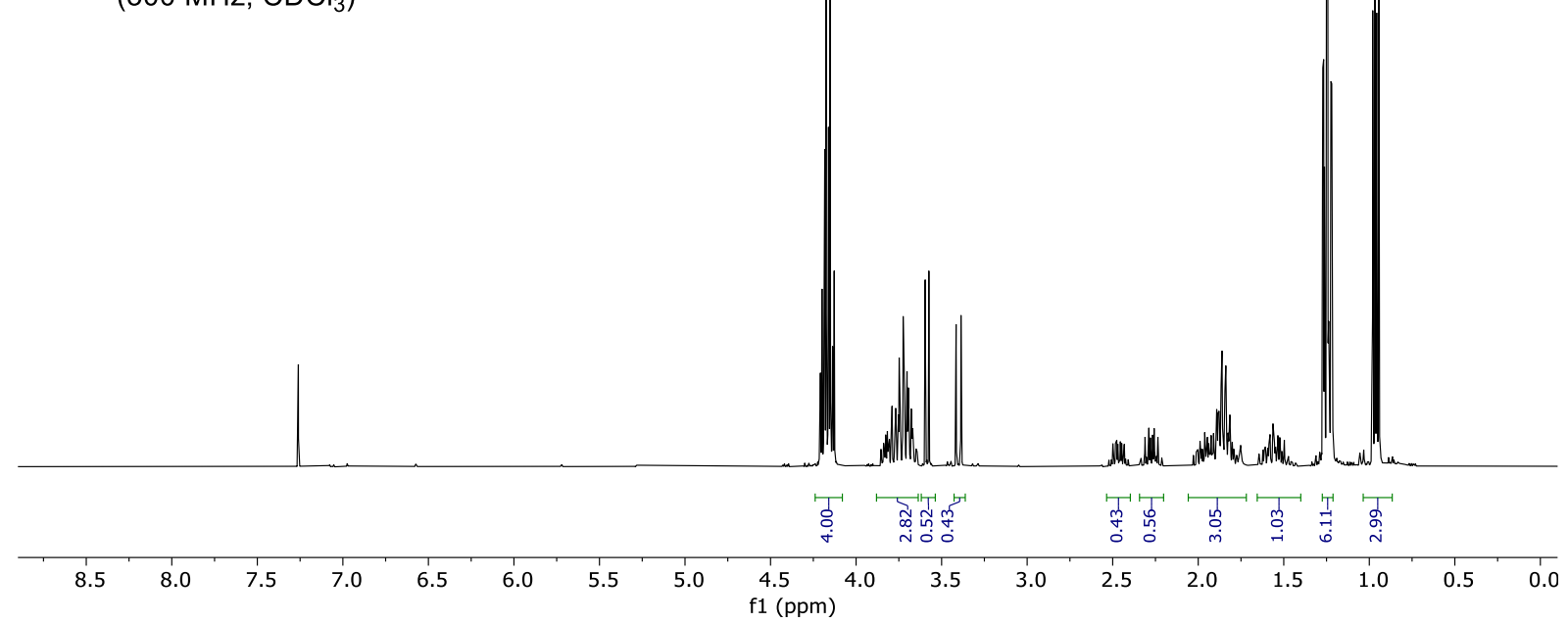

$\mathrm{CO}_{2} \mathrm{Et}$<smiles>CCOC(=O)CC(C)C1CCCO1</smiles>

8sa (a)

${ }^{13} \mathrm{C}$ NMR $\left(75 \mathrm{MHz}, \mathrm{CDCl}_{3}\right)$

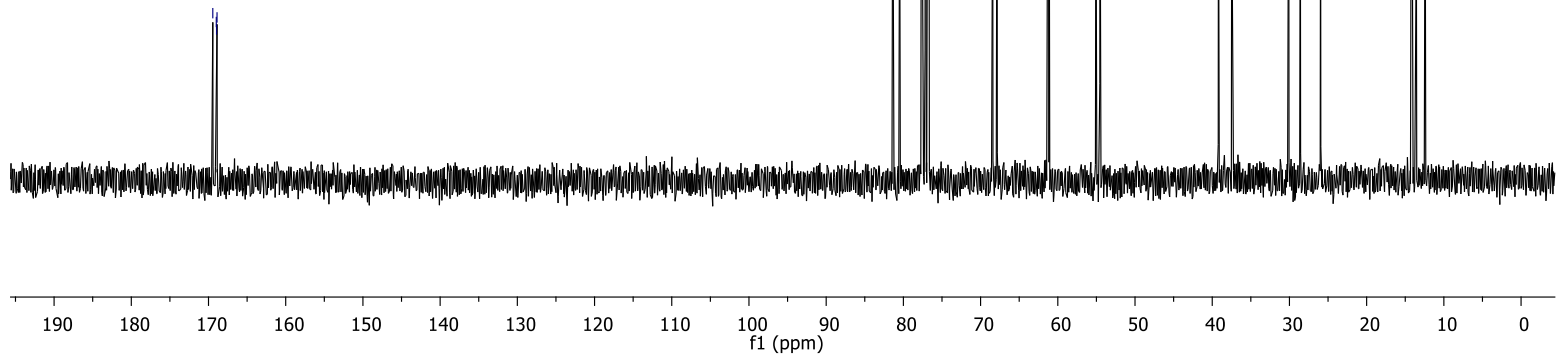

Figure S23. 1H- and 13C NMR spectra of 8sa (a). 


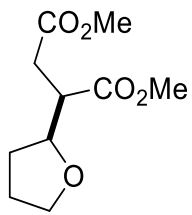

8sa (b)

${ }^{1} \mathrm{H}$ NMR

$\left(300 \mathrm{MHz}, \mathrm{CDCl}_{3}\right)$

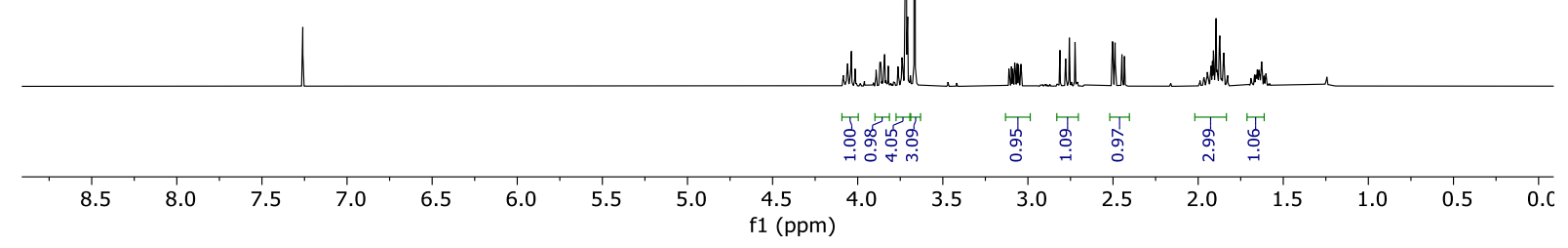

il<smiles>CC(=O)CC(C(C)=O)C1CCCO1</smiles>

8sa (b)

${ }^{13} \mathrm{C}$ NMR

(75 $\mathrm{MHz}, \mathrm{CDCl} 3)$
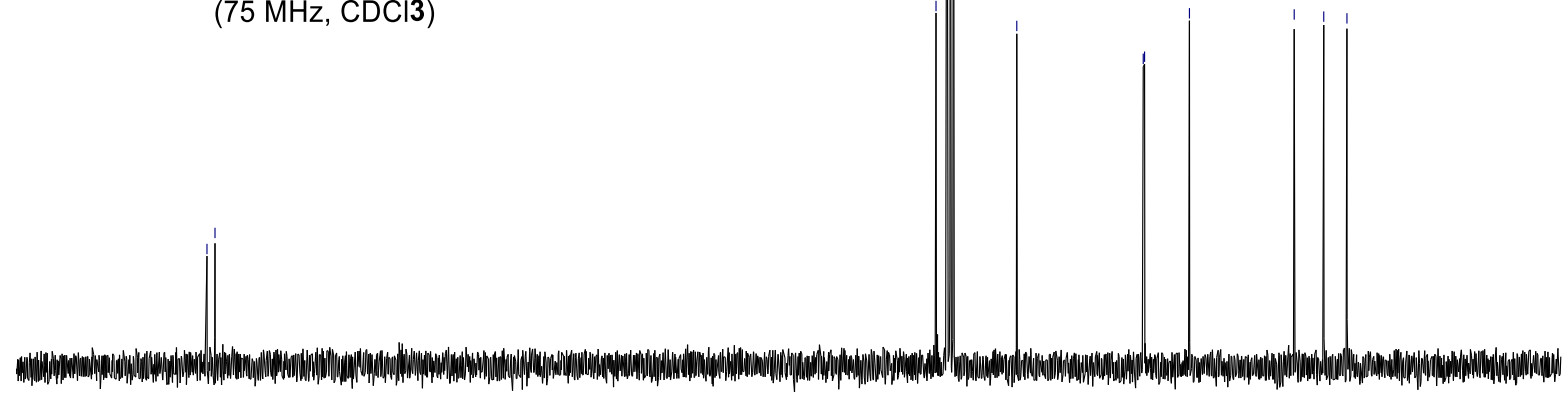

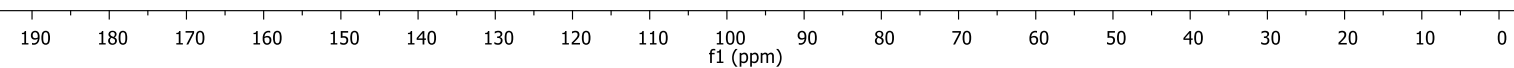

Figure S24. 1H- and 13C NMR spectra of 8sa (b). 
$\mathrm{EtO}_{2} \mathrm{C}$<smiles>CCOC(=O)C(CC)C(C)C1CCC1</smiles>

8tb

${ }^{1} \mathrm{H}$ NMR

$\left(300 \mathrm{MHz}, \mathrm{CDCl}_{3}\right)$

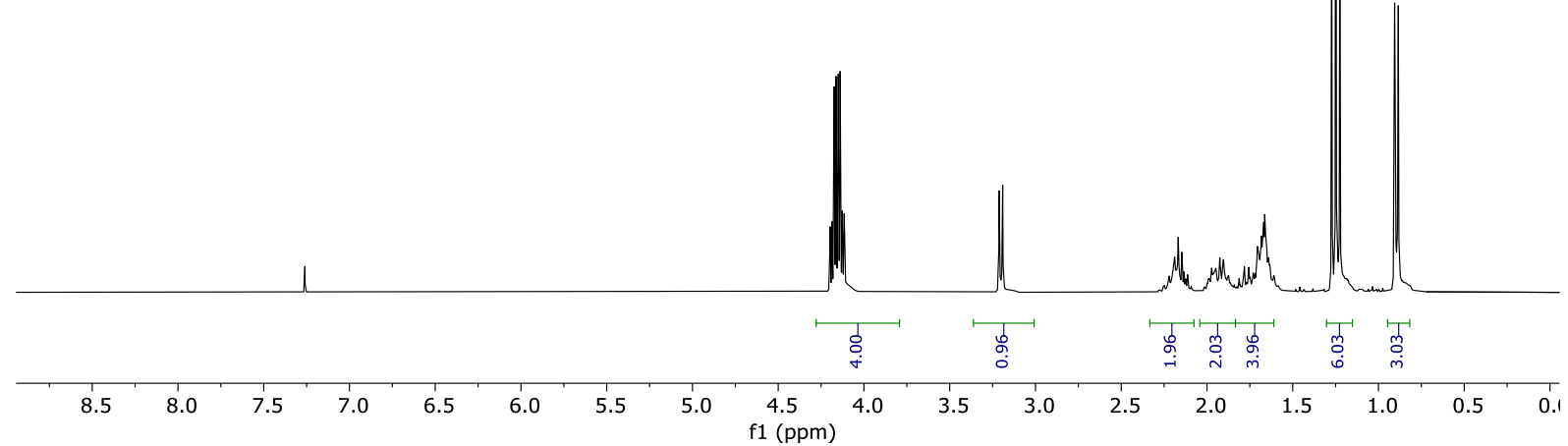

$\mathrm{EtO}_{2} \mathrm{C}$

$-\mathrm{CO}_{2} \mathrm{Et}$<smiles>C1CC2CC12</smiles>

8tb

${ }^{13} \mathrm{C}$ NMR

$\left(75 \mathrm{MHz}, \mathrm{CDCl}_{3}\right)$

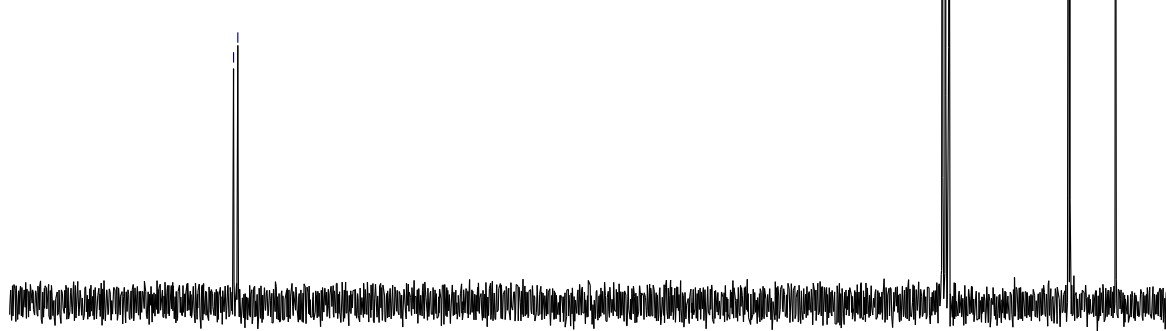

$90 \quad 180 \quad 170 \quad 160$
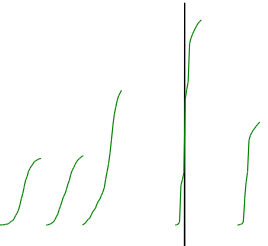

Y.
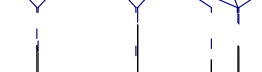


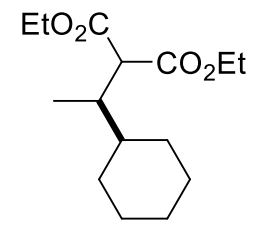

$8 u b$

${ }^{1} \mathrm{H}$ NMR

$\left(300 \mathrm{MHz}, \mathrm{CDCl}_{3}\right)$

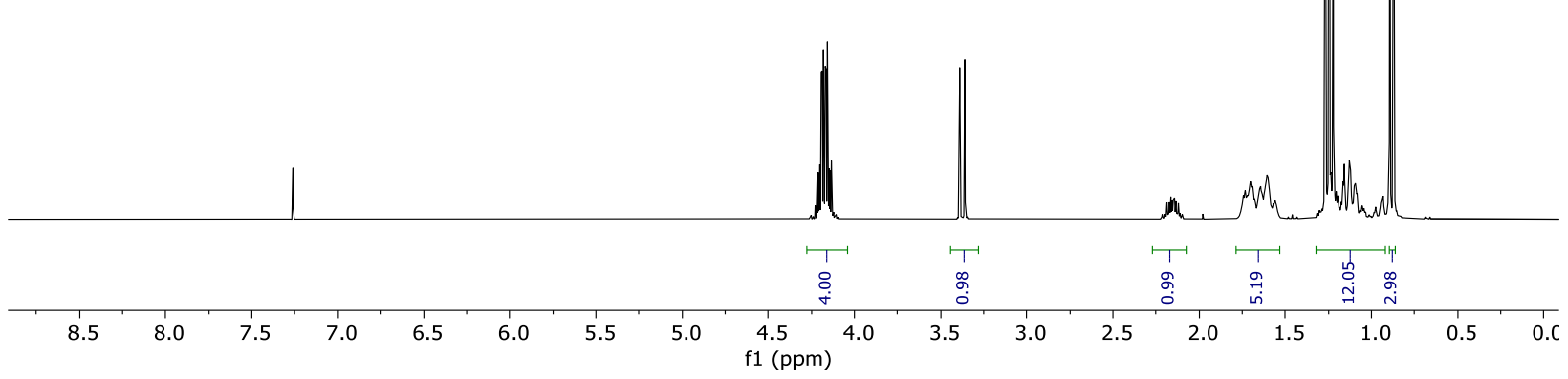

$\mathrm{EtO}_{2} \mathrm{C}$

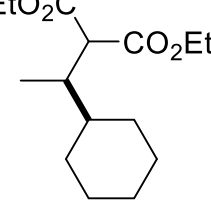

$8 u b$

${ }^{13} \mathrm{C} N M R$

(75 $\mathrm{MHz}, \mathrm{CDCl}_{3}$ )

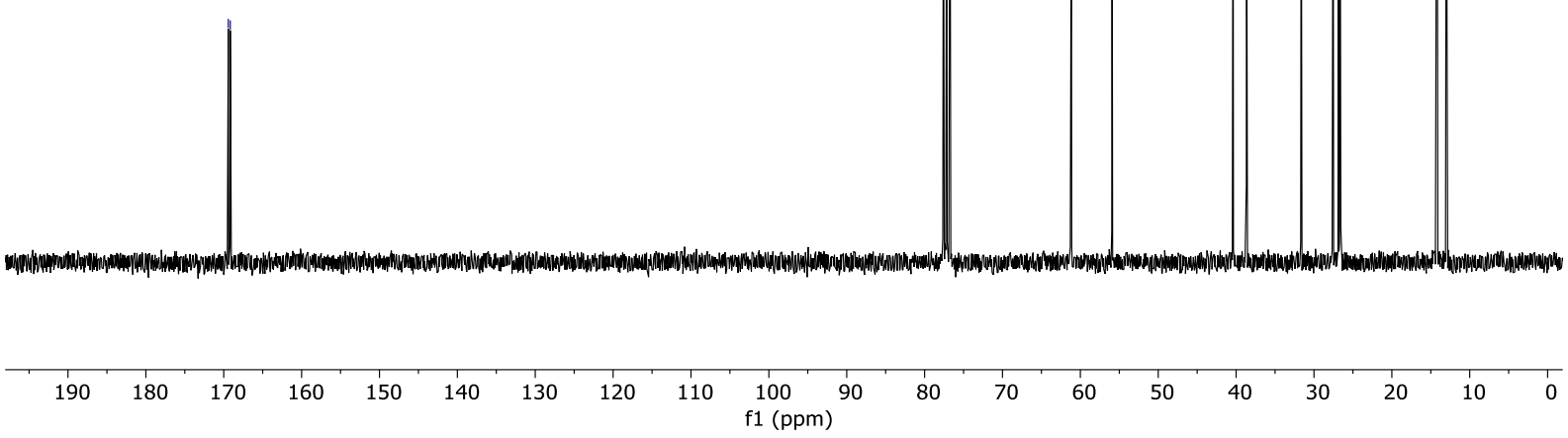

Figure S26. $1 \mathrm{H}$ - and 13C NMR spectra of 8ub. 

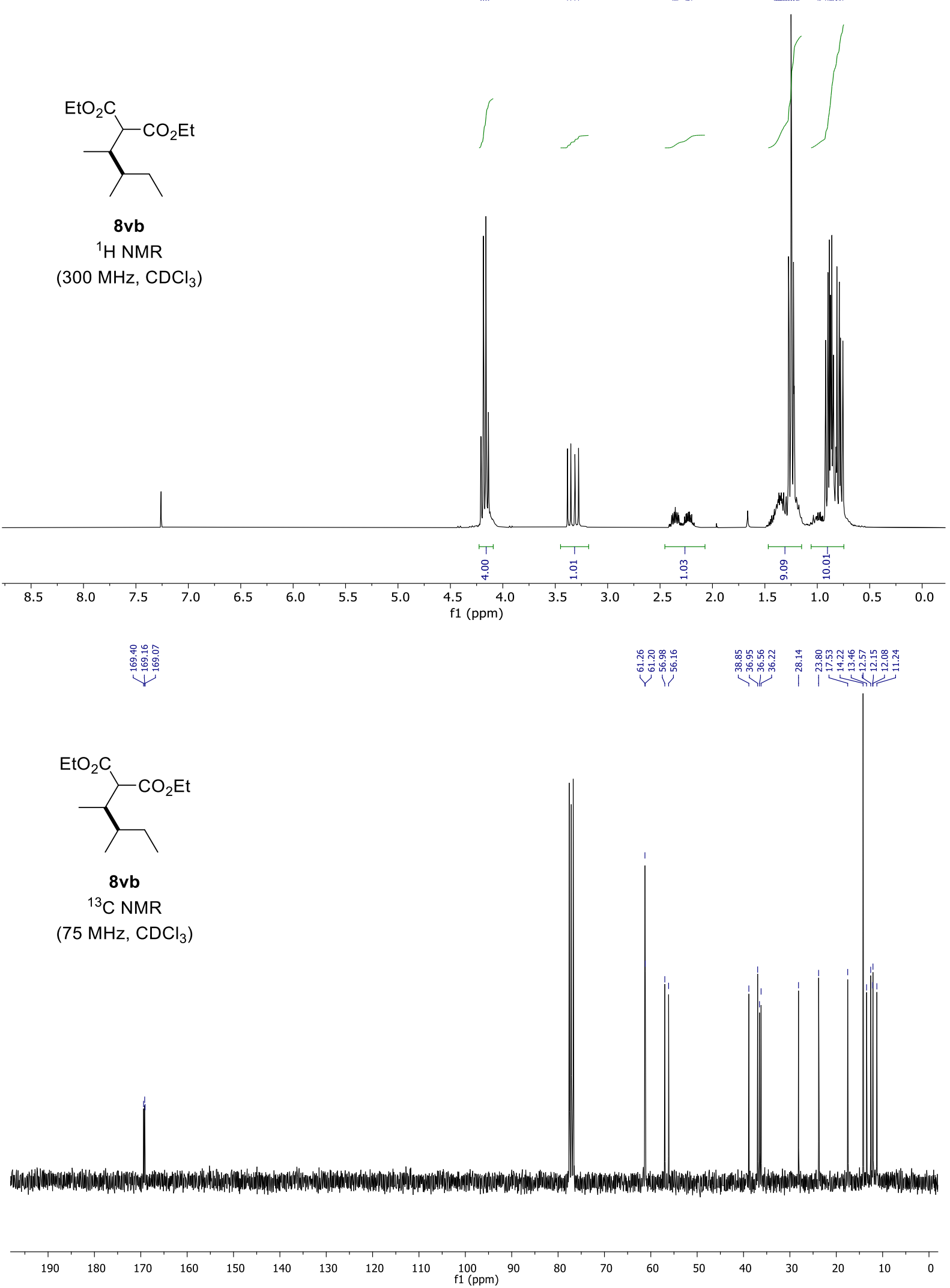

Figure $\mathbf{S 2 7} .1 \mathrm{H}$ - and $13 \mathrm{C}$ NMR spectra of $8 \mathrm{vb}$. 
<smiles>CCOC(=O)NCC(C(=O)OCC)C(C)CC</smiles>
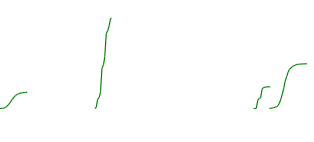

$8 w b$

${ }^{1} \mathrm{H}$ NMR

(300 MHz, $\mathrm{CDCl}_{3}$ )

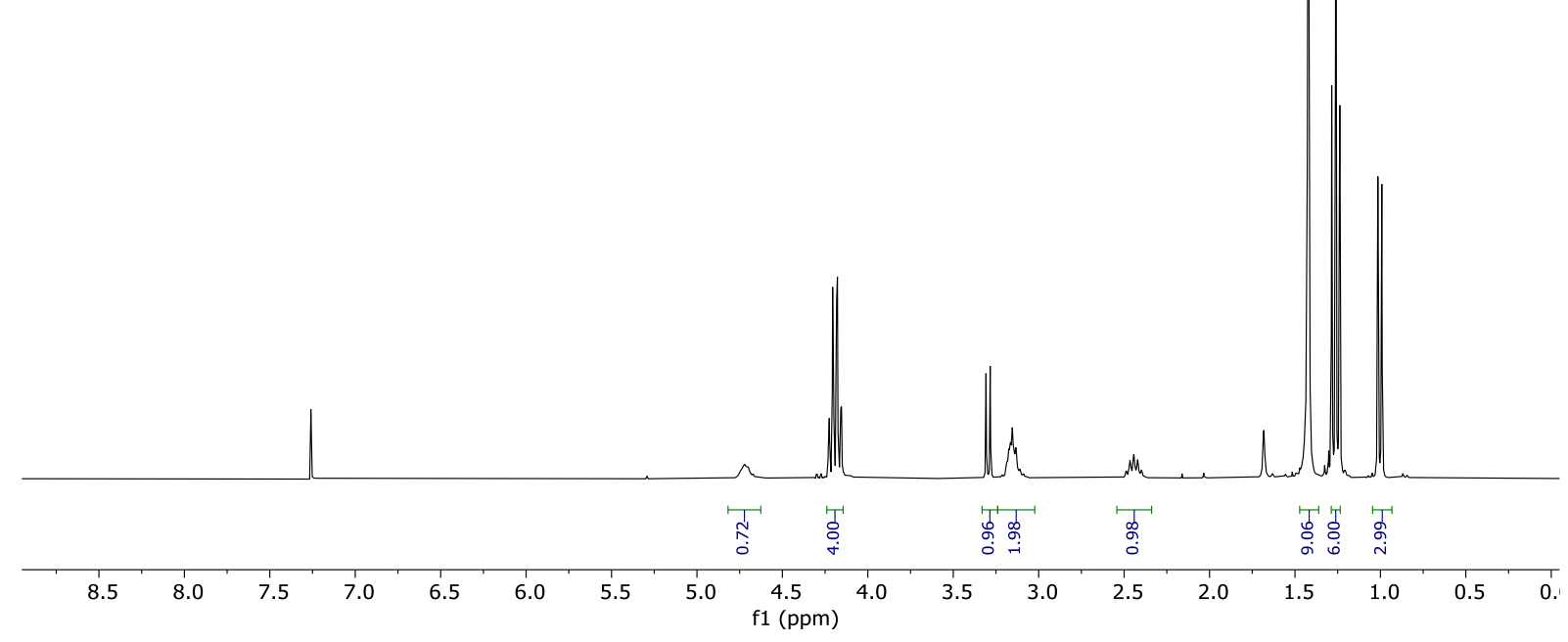<smiles>CCOC(=O)OCCNC(C)C(C)C(=O)OCC</smiles>

$8 w b$

${ }^{13} \mathrm{C} \mathrm{NMR}$

$\left(75 \mathrm{MHz}, \mathrm{CDCl}_{3}\right)$

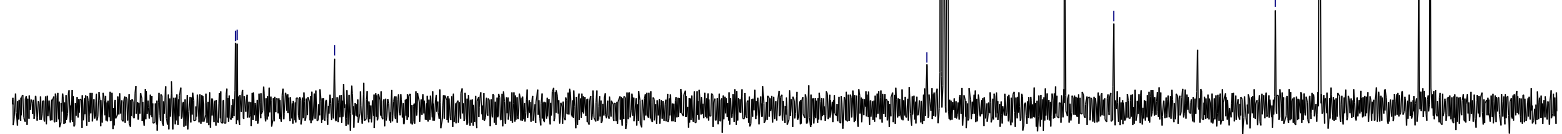

Figure S28. $1 \mathrm{H}$ - and $13 \mathrm{C}$ NMR spectra of $\mathbf{8 w b}$. 
<smiles>CCOC(=O)C(C)COC1CCCCC1</smiles>

$8 x b$

${ }^{1} \mathrm{H} N M R$

$\left(300 \mathrm{MHz}, \mathrm{CDCl}_{3}\right)$

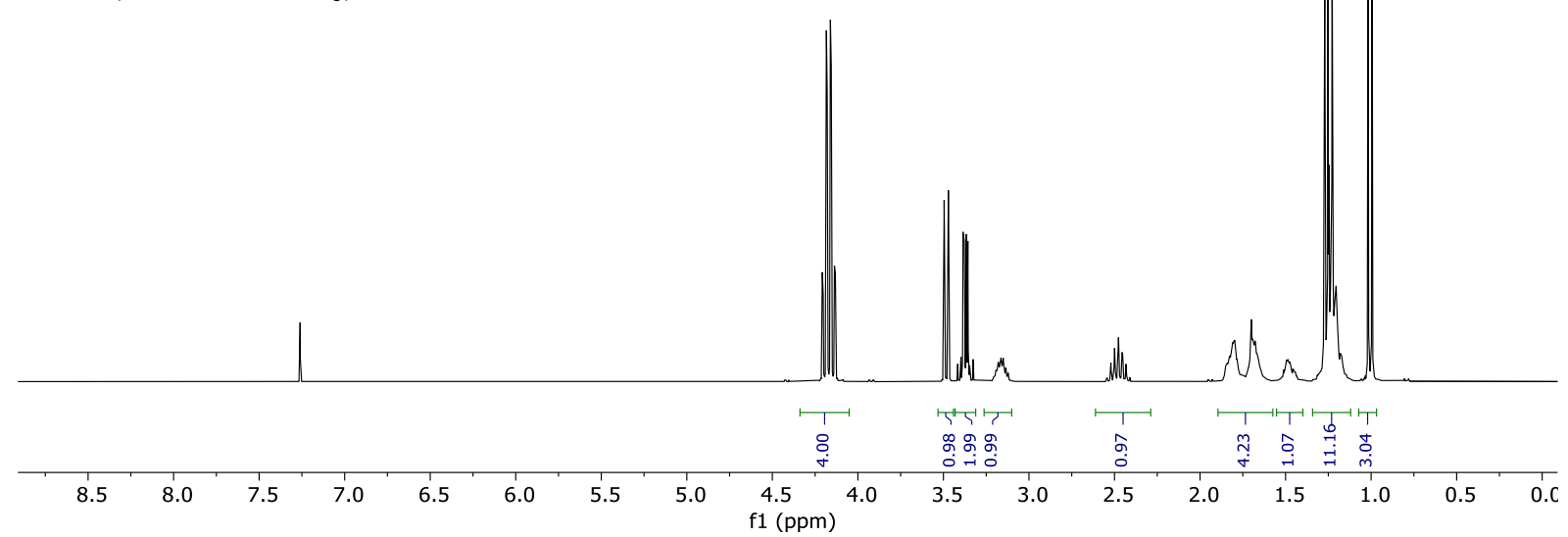<smiles>CCOC(=O)C(C)C(C)COC1CCCCC1</smiles>

$8 \times b$

${ }^{13} \mathrm{C}$ NMR

(300 $\mathrm{MHz}, \mathrm{CDCl}_{3}$ )

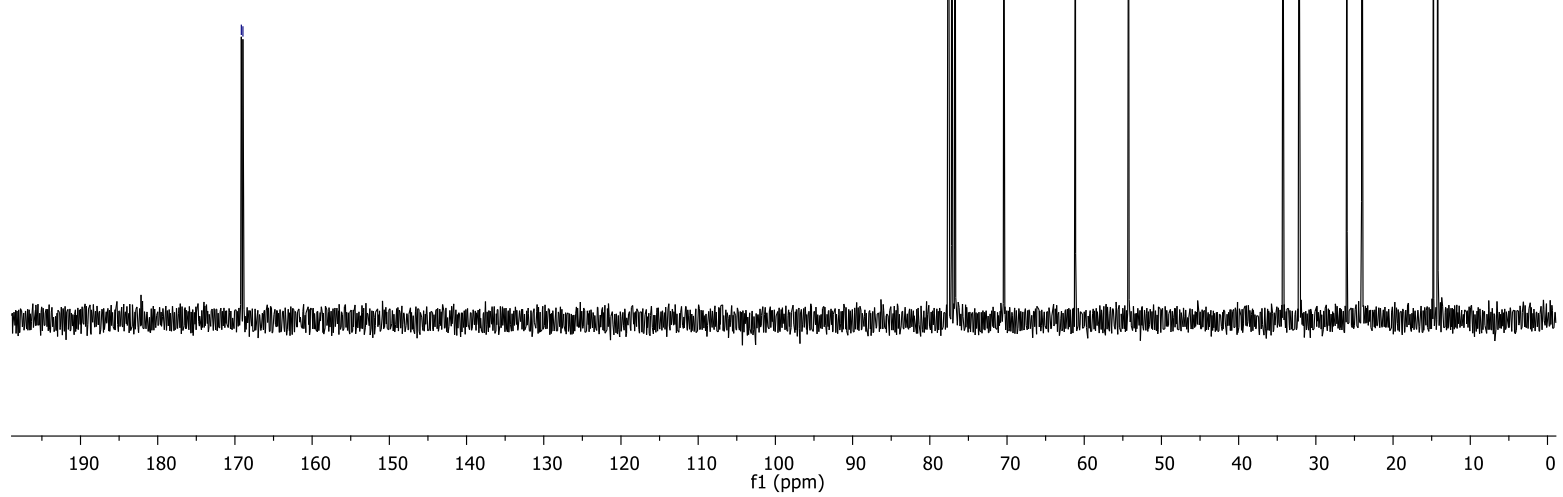

Figure S29. $1 \mathrm{H}$ - and 13C NMR spectra of $\mathbf{8 x b}$. 


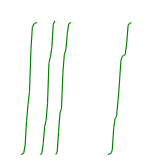<smiles>CCc1ccc(-c2ccccc2)c(F)c1</smiles>

9

${ }^{1} \mathrm{H}$ NMR

$\left(300 \mathrm{MHz}, \mathrm{CDCl}_{3}\right)$
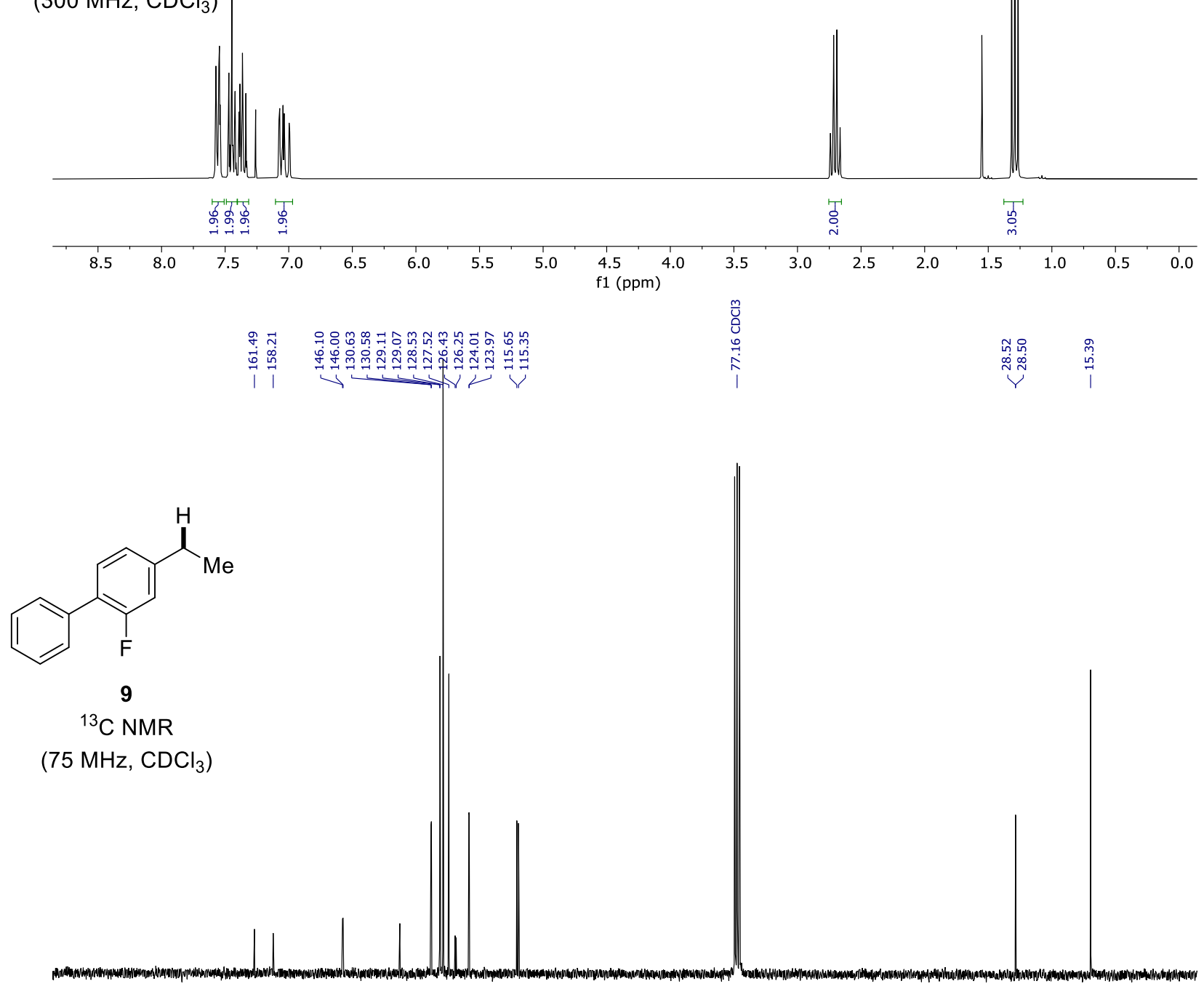

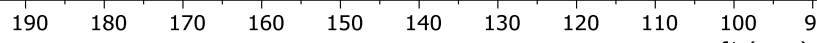

Figure S30. 1H- and 13C NMR spectra of 9. 


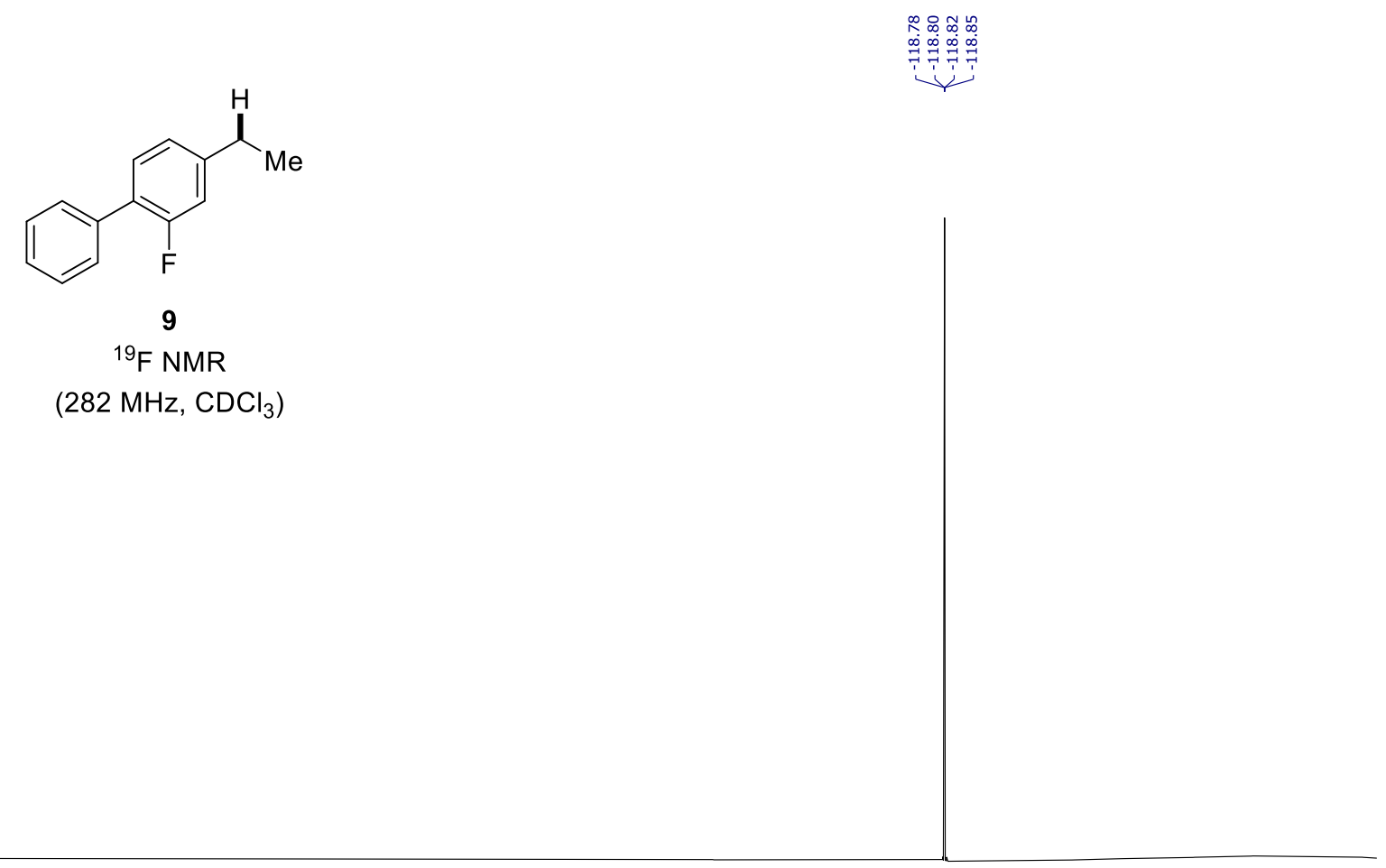

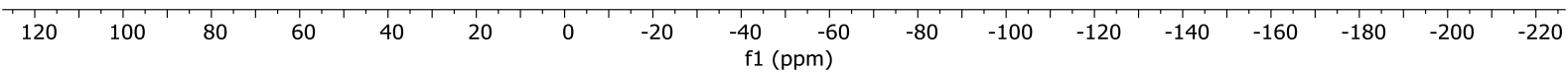

Figure S31. 19F-NMR spectrum of 9. 
<smiles>O=C1CCC(C23CC4CC(CC(C4)C2)C3)C1</smiles>

10

${ }^{1} \mathrm{H}$ NMR

(400 MHz, $\mathrm{CDCl}_{3}$ )

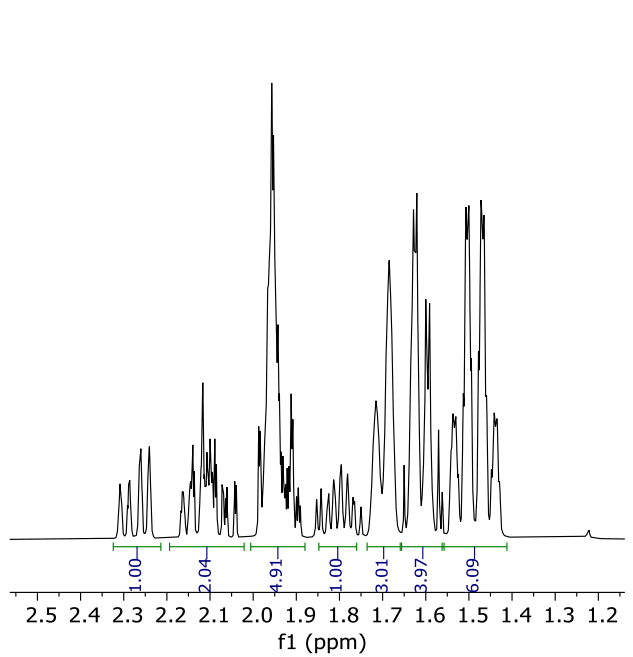

f1 (ppm)
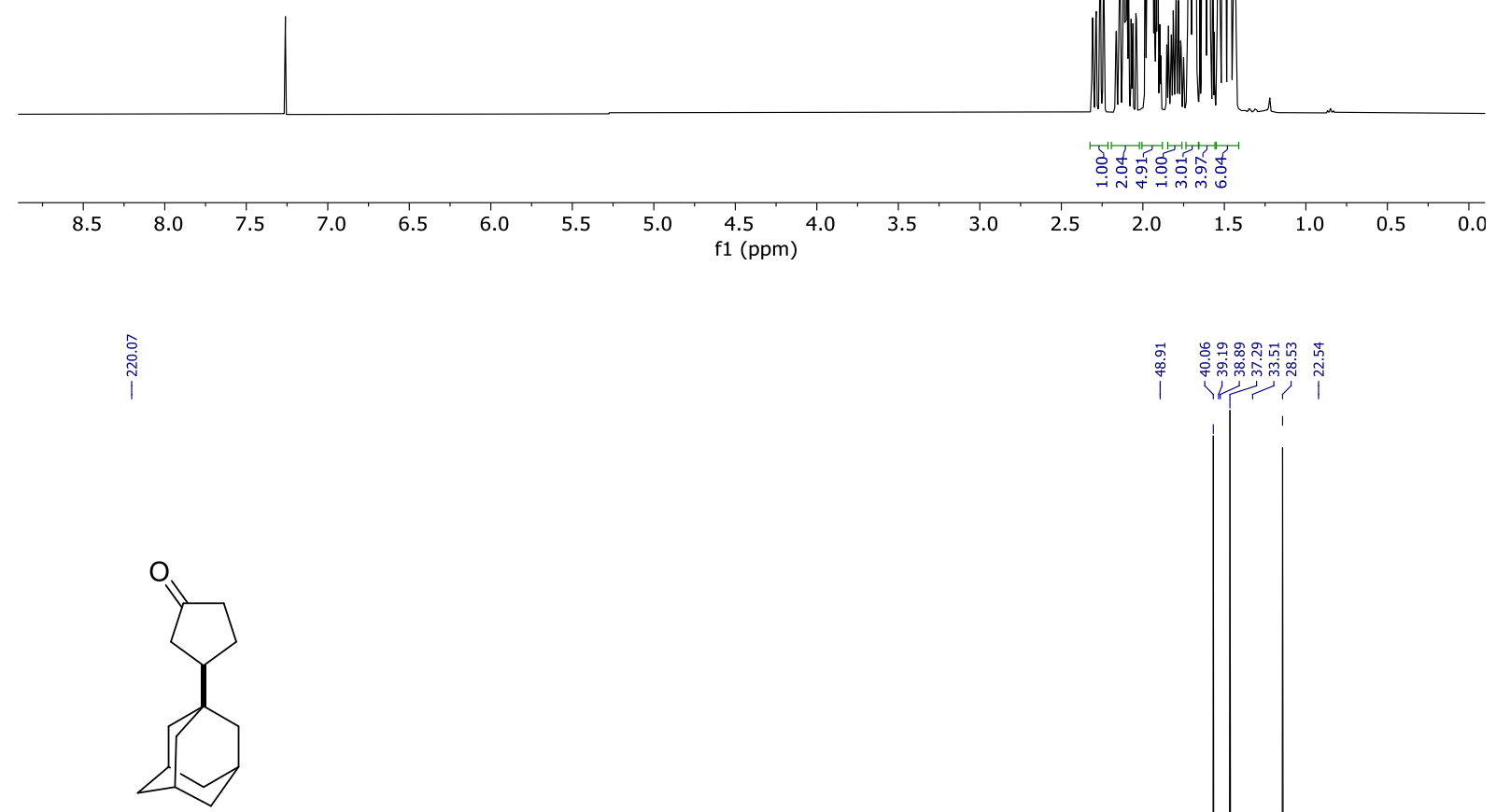

10

${ }^{13} \mathrm{C}$ NMR

(101 MHz, $\mathrm{CDCl}_{3}$ )
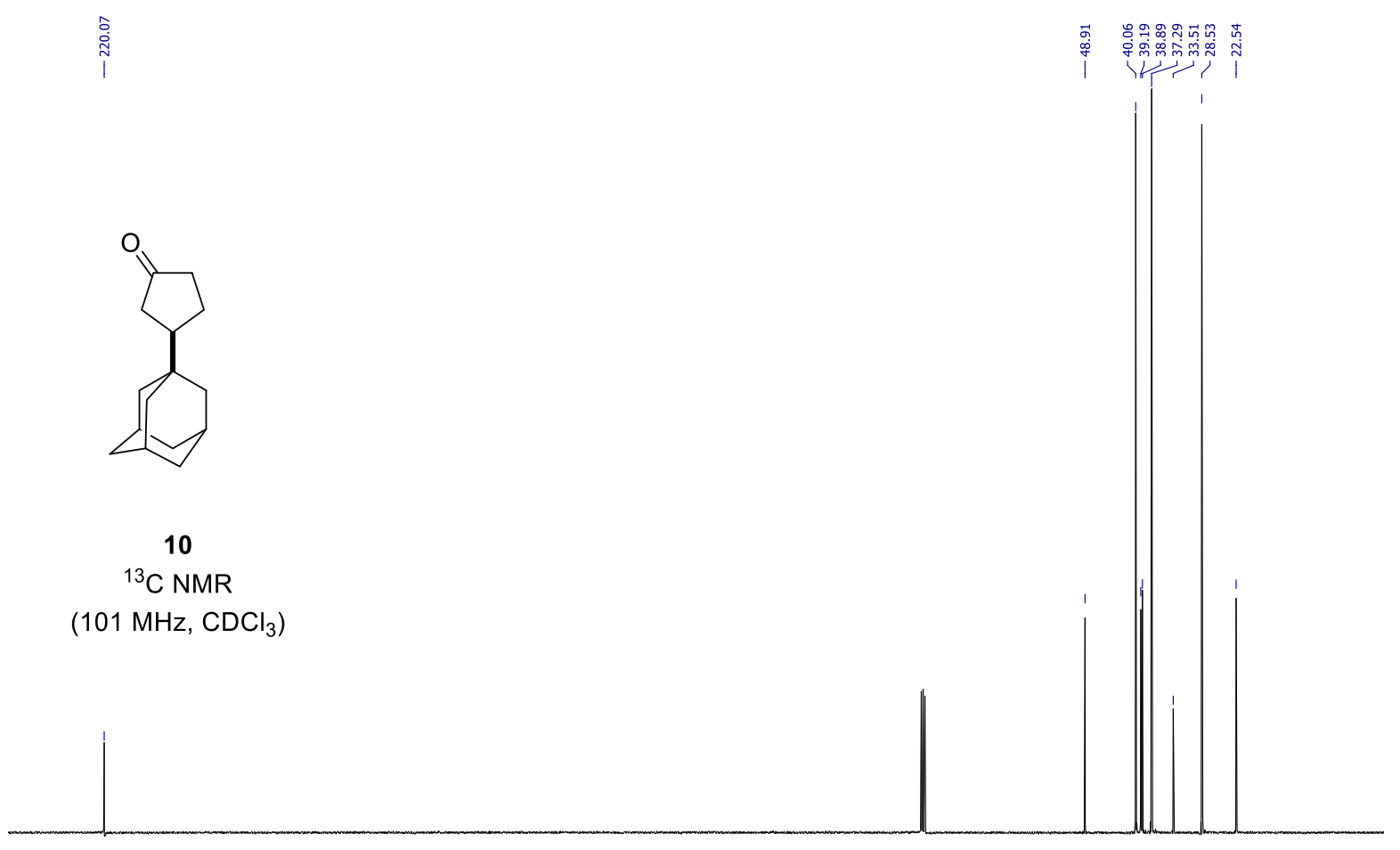

$\begin{array}{llllllllllllllllllllllllllllll}230 & 220 & 210 & 200 & 190 & 180 & 170 & 160 & 150 & 140 & 130 & 120 & 110 & 100 & 90 & 80 & 70 & 60 & 50 & 40 & 30 & 20 & 10 & 0\end{array}$

Figure S32. $1 \mathrm{H}$ - and 13C NMR spectra of 10. 


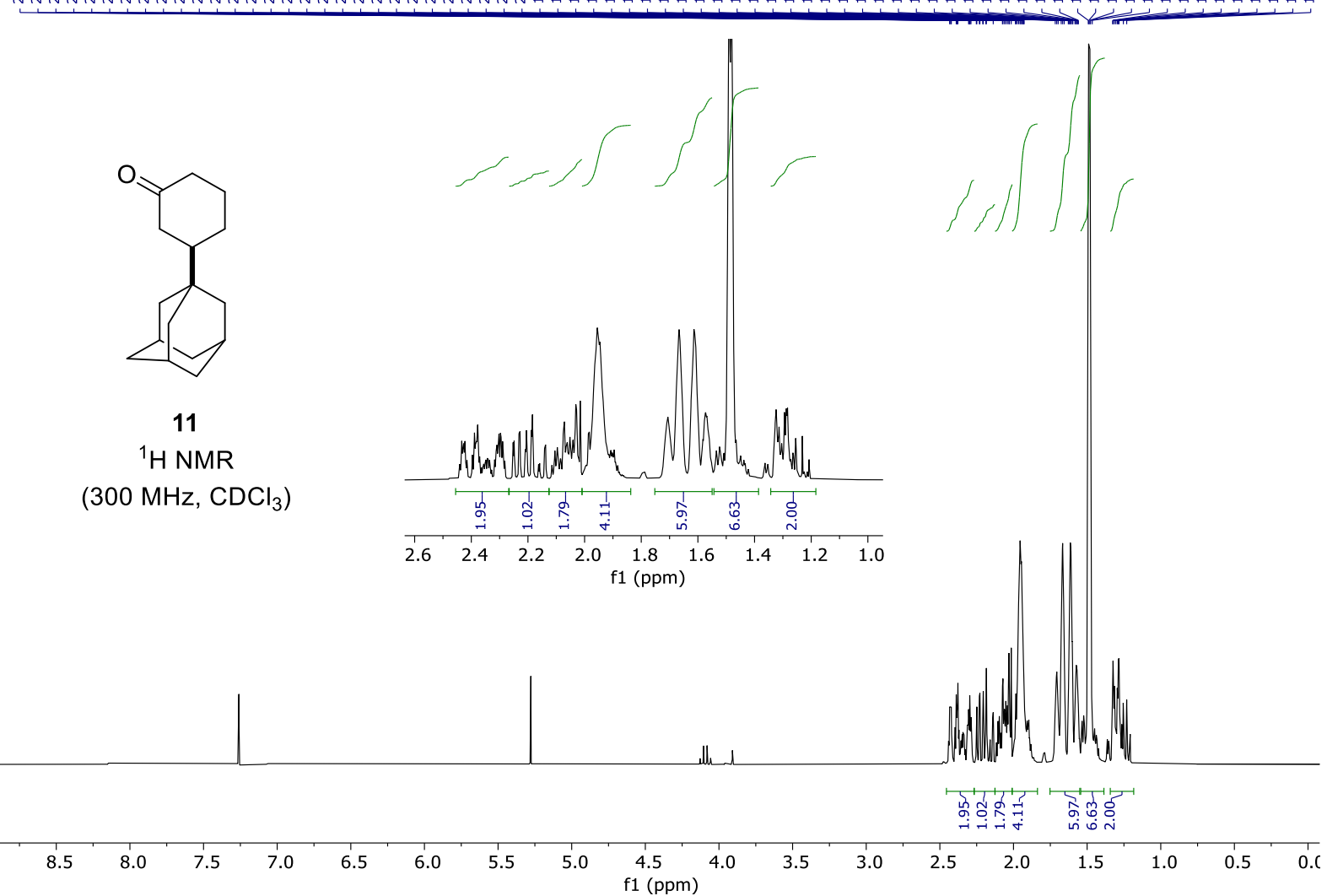

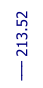<smiles>O=C1CCC[C@H](C23CC4CC(CC(C4)C2)C3)C1</smiles>

11

${ }^{13} \mathrm{C}$ NMR

$\left(75 \mathrm{MHz}, \mathrm{CDCl}_{3}\right)$

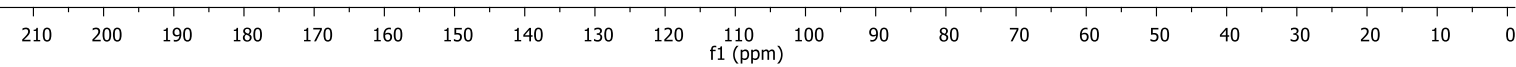

Figure S33. 1H- and 13C NMR spectra of 11. 


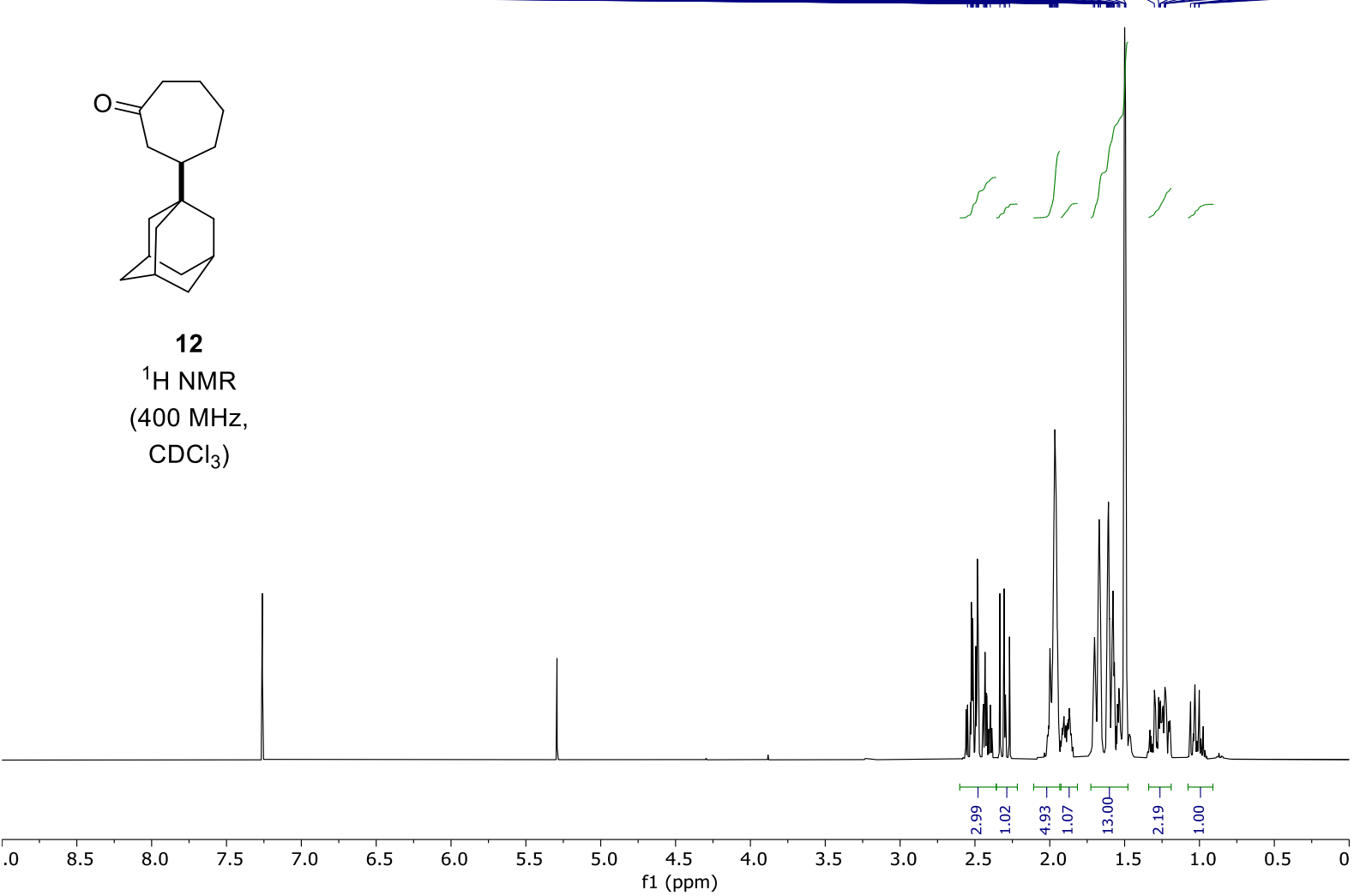

$\underset{\substack{\dot{0} \\ \text { I }}}{\stackrel{0}{1}}$

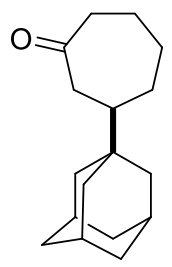

12

${ }^{13} \mathrm{C}$ NMR

$\left(101 \mathrm{MHz}, \mathrm{CDCl}_{3}\right)$

$\begin{array}{lllllllllll}220 & 210 & 200 & 190 & 180 & 170 & 160 & 150 & 140 & 130 & 120 \\ \mathrm{f} 1(\mathrm{ppm})\end{array}$

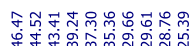

1811/
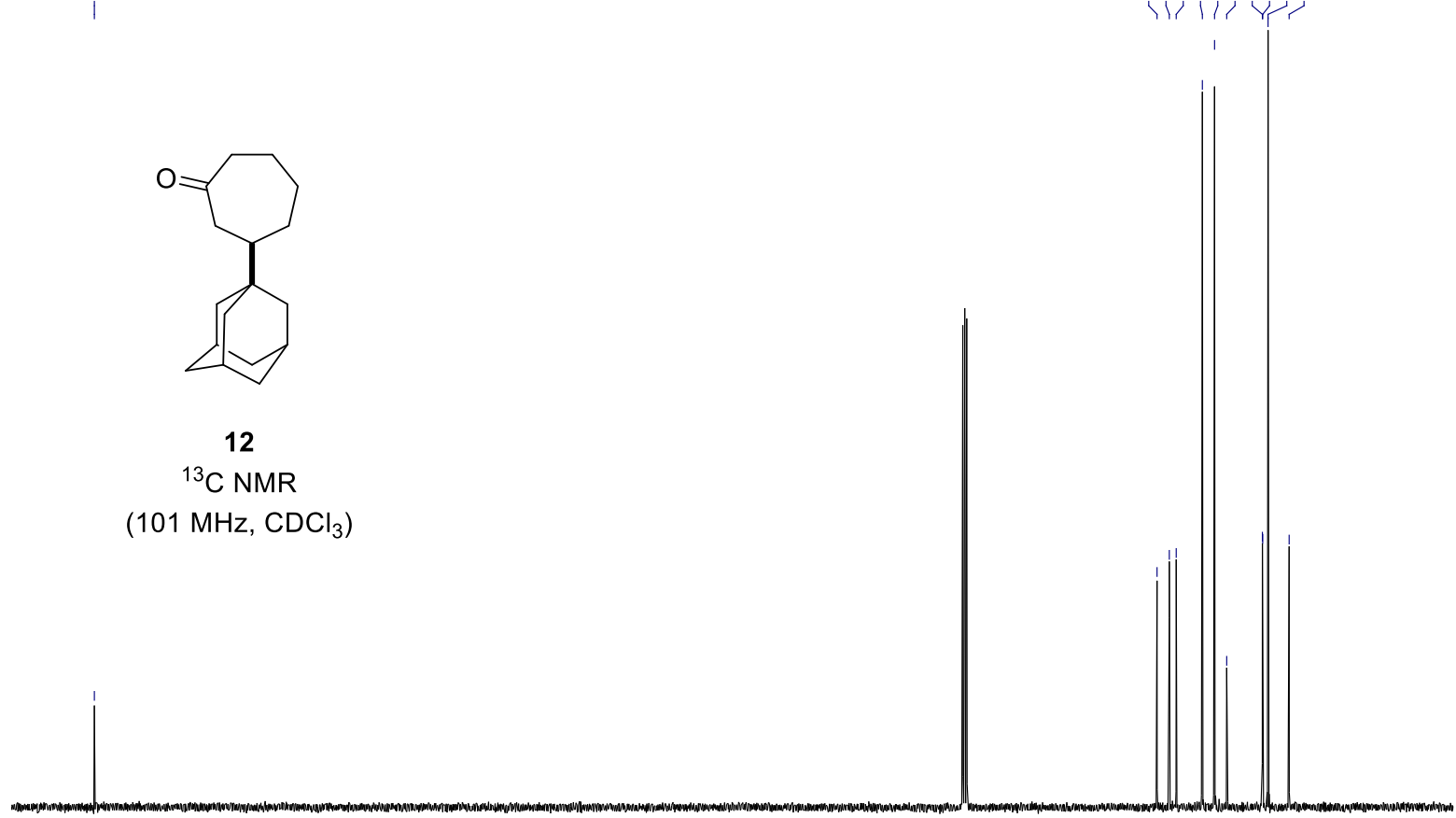

Figure S34. 1H- and 13C NMR spectra of 12. 


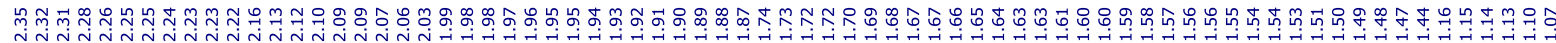<smiles>CC1C(=O)CCC1C1CC2CCC(C2)C1</smiles>

13

${ }^{1} \mathrm{H}$ NMR

$\left(300 \mathrm{MHz}, \mathrm{CDCl}_{3}\right)$

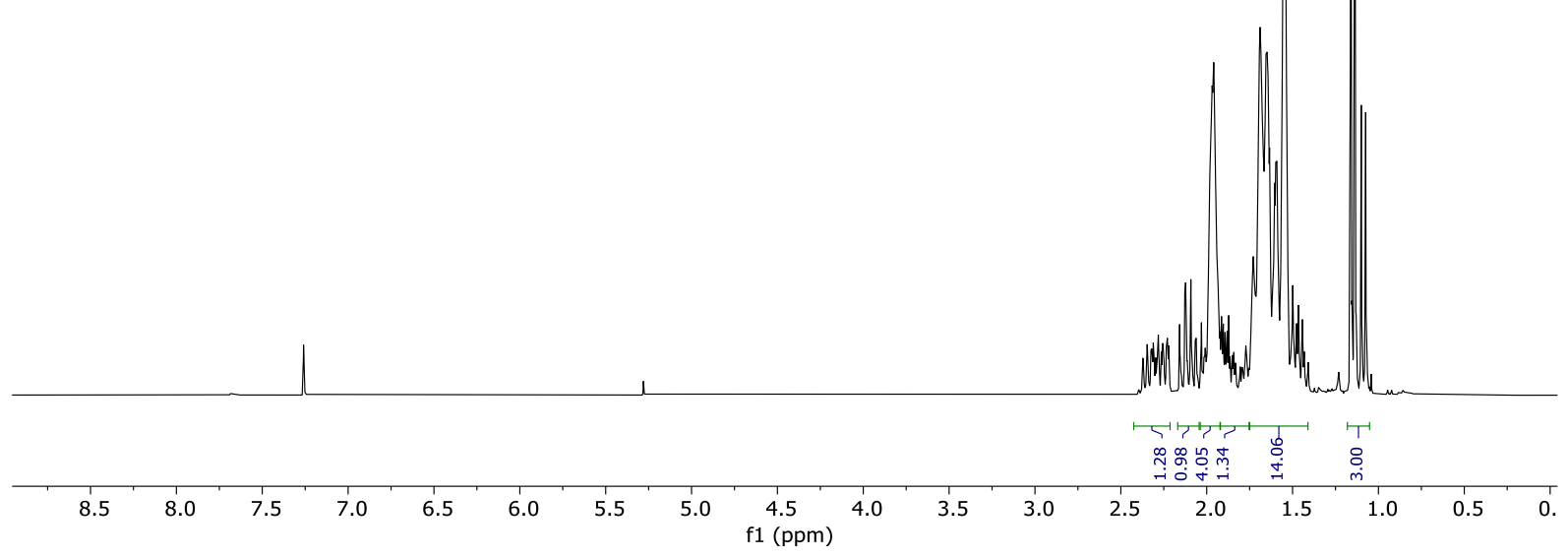

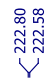

il<smiles>CC1C(=O)CCC1C1CC2CCC(C2)C1</smiles>

13

${ }^{13} \mathrm{C}$ NMR

(75 $\mathrm{MHz}, \mathrm{CDCl}_{3}$ )

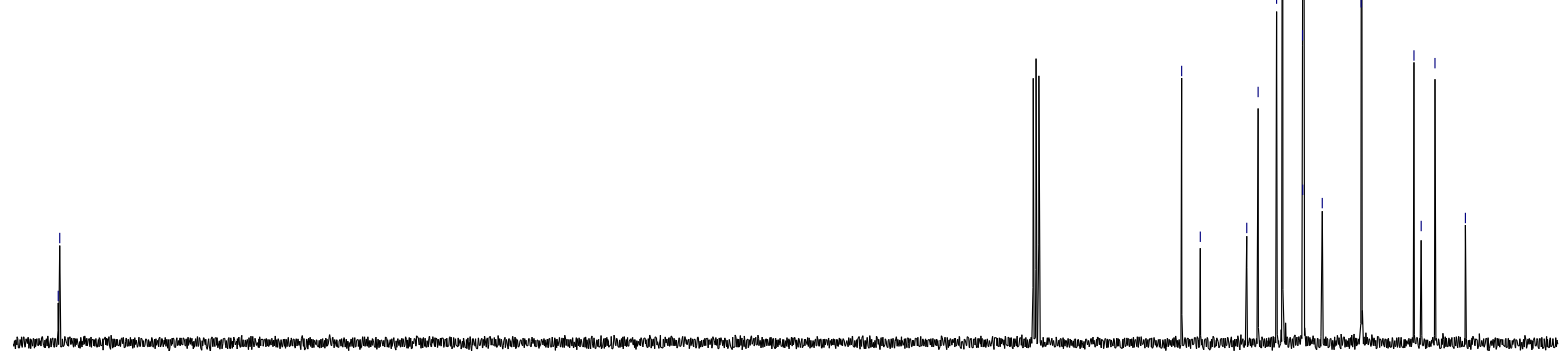

$\begin{array}{lllllllllll}220 & 210 & 200 & 190 & 180 & 170 & 160 & 150 & 140 & 130 & 120 \\ \mathrm{f} 1(\mathrm{ppm})\end{array}$

Figure S35. 1H- and 13C NMR spectra of 13. 

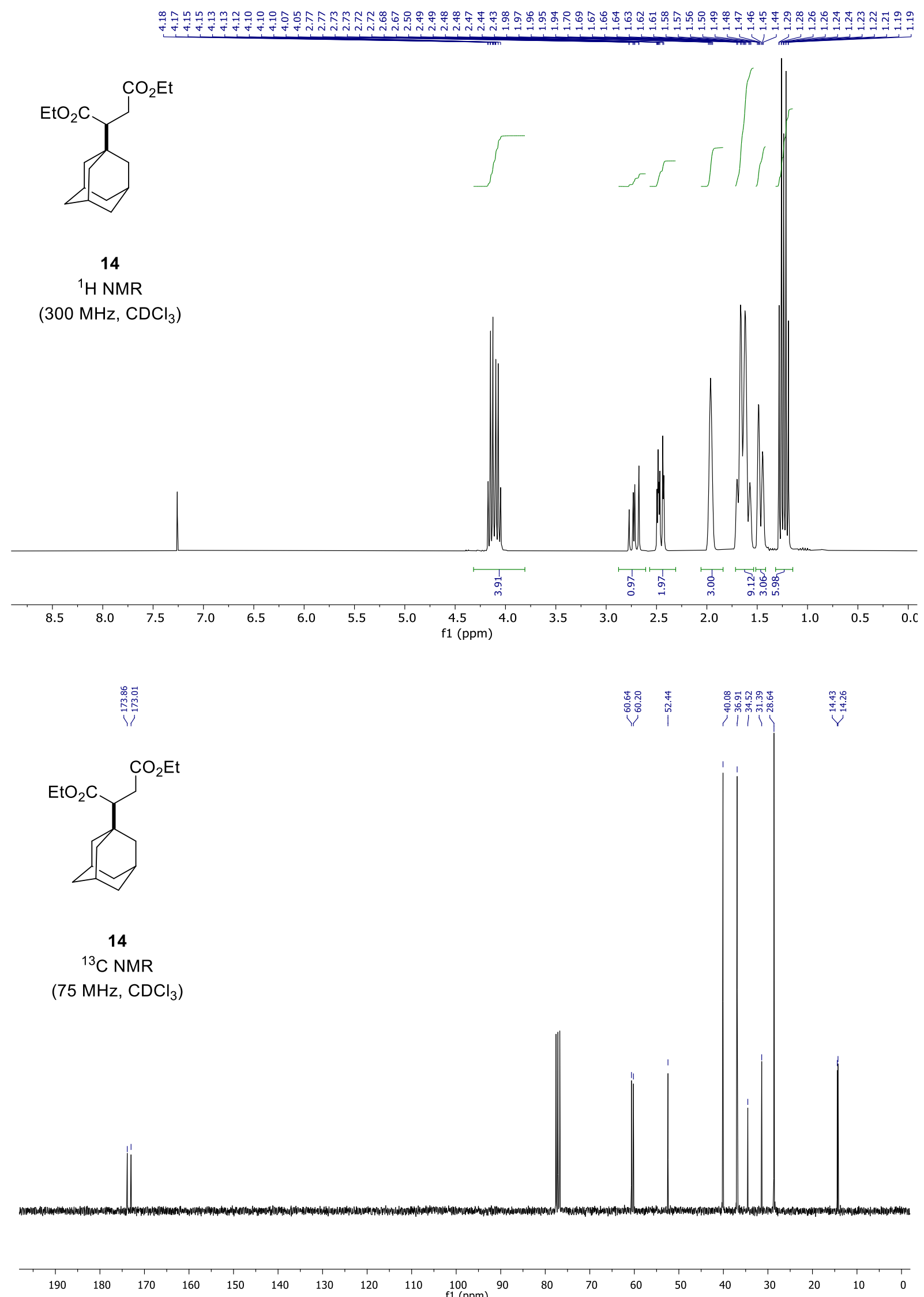

Figure S36. $1 \mathrm{H}$ - and 13C NMR spectra of 14 . 


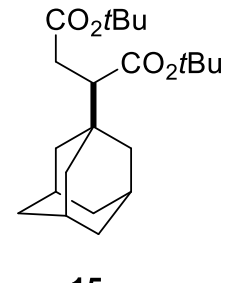

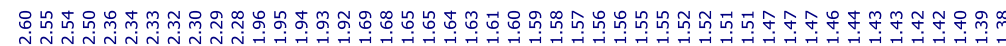

15

${ }^{1} \mathrm{H}$ NMR

$\left(300 \mathrm{MHz}, \mathrm{CDCl}_{3}\right)$

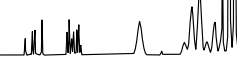

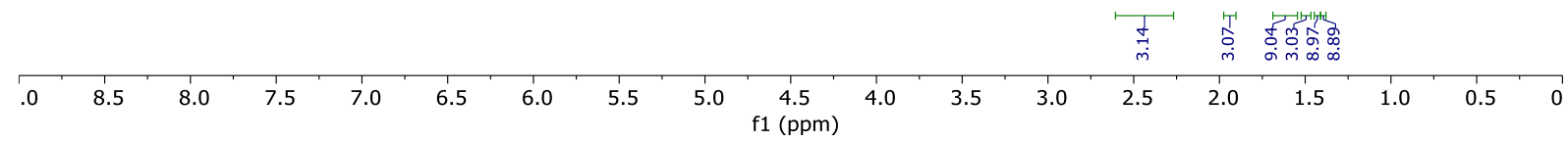

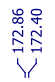

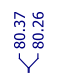

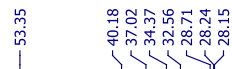

$\mathrm{CO}_{2} \mathrm{tBu}$

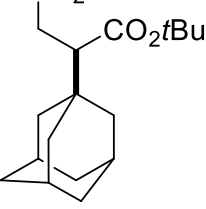

15

${ }^{13} \mathrm{C}$ NMR

(75 MHz, $\mathrm{CDCl}_{3}$ )

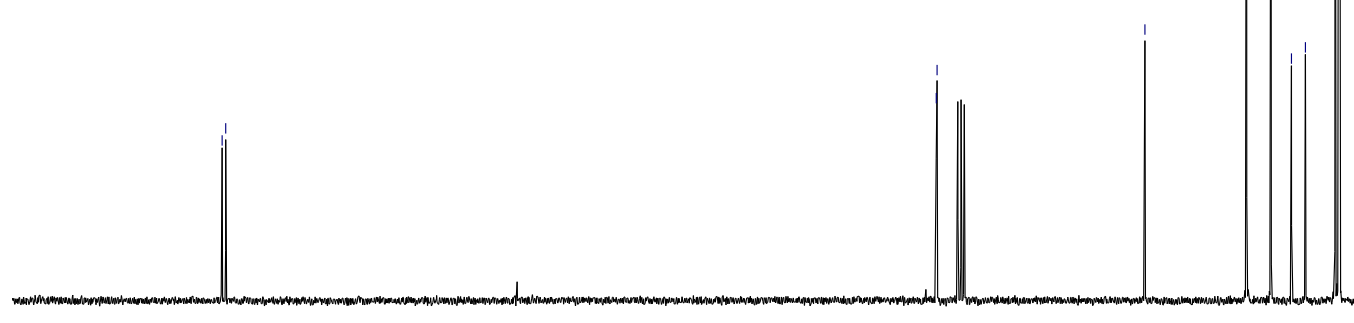

200

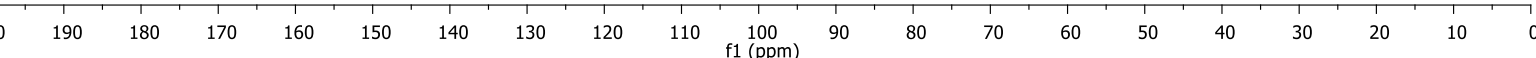

Figure S37. 1H- and 13C NMR spectra of 15. 


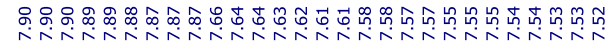

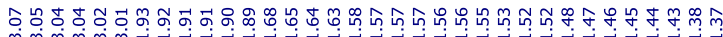
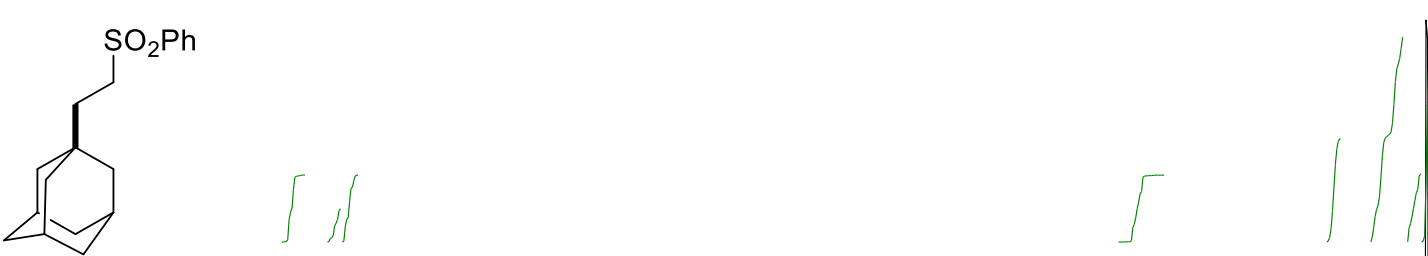

16

${ }^{1} \mathrm{H}$ NMR

(300 $\mathrm{MHz}, \mathrm{CDCl}_{3}$ )
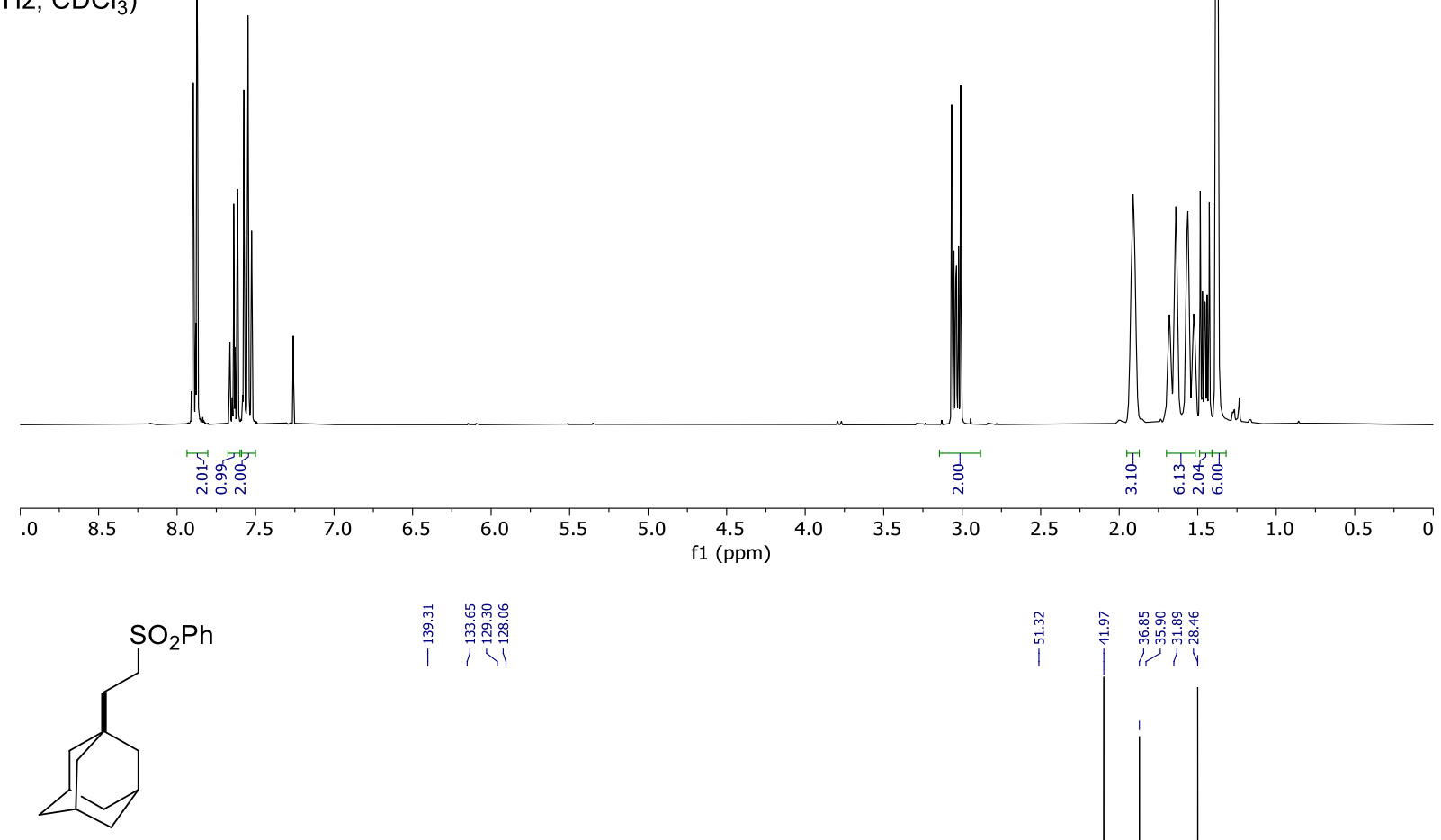

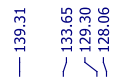

16

${ }^{13} \mathrm{C}$ NMR

$\left(75 \mathrm{MHz}, \mathrm{CDCl}_{3}\right.$ )

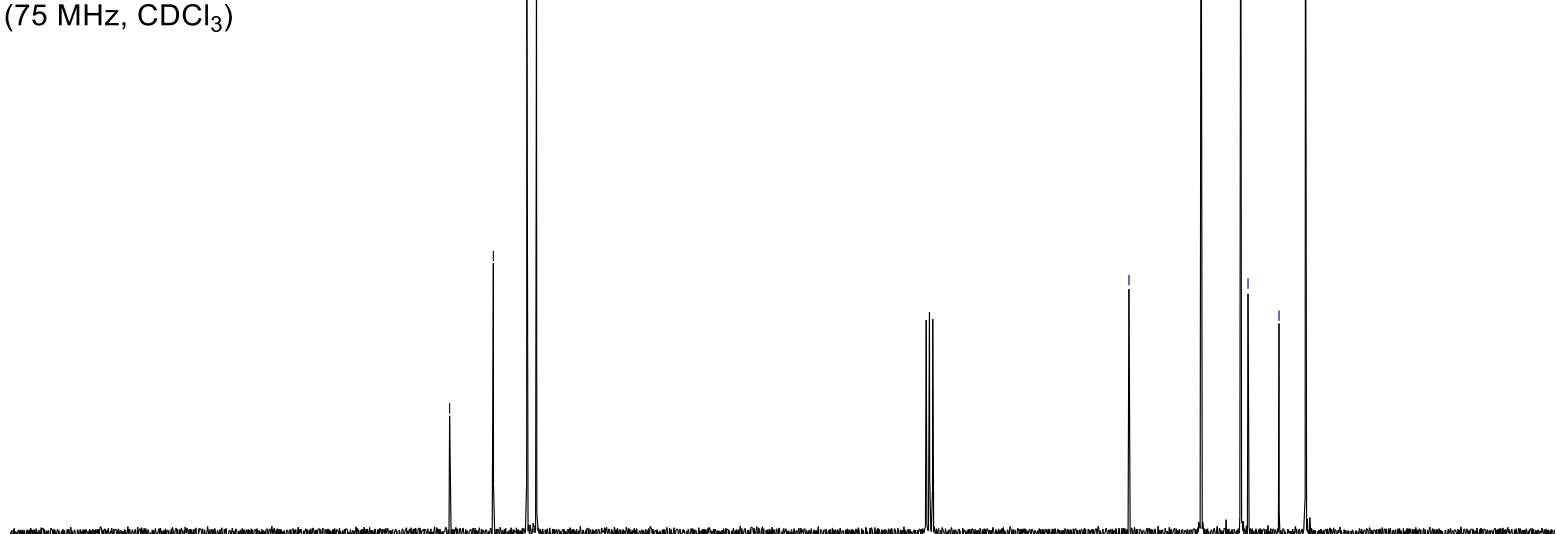

190

$\begin{array}{llll}170 & 160 & 150 & 140\end{array}$

| 

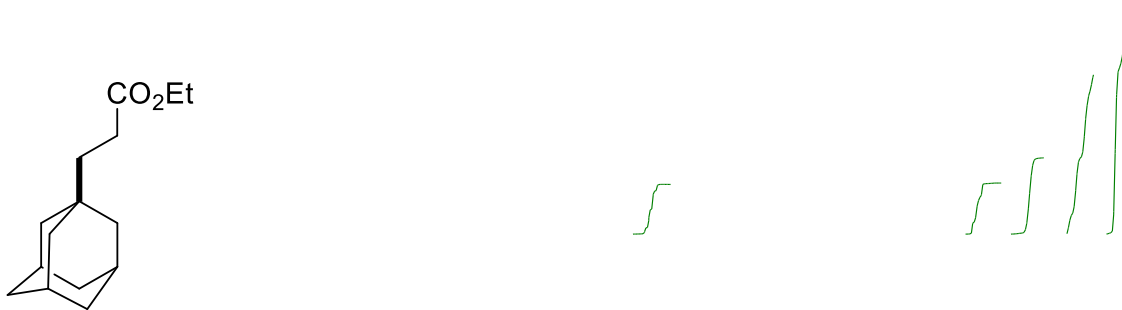

17

${ }^{1} \mathrm{H}$ NMR

(300 MHz, $\mathrm{CDCl}_{3}$ )

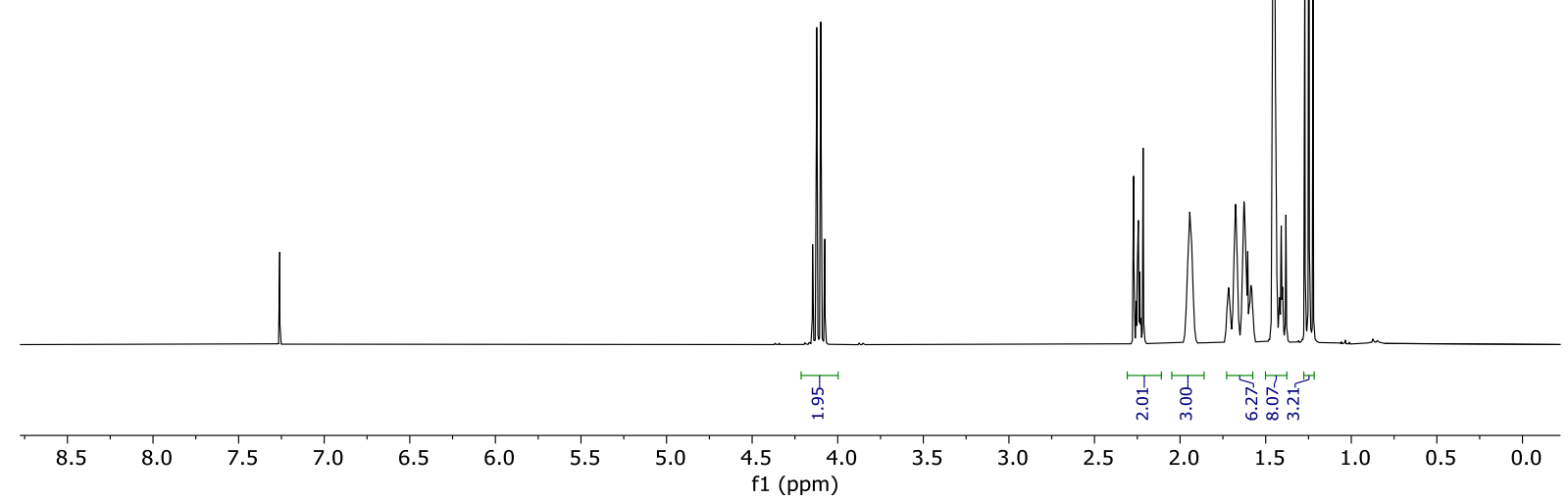

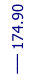<smiles>CCOC(=O)CCC12CC3CC(CC(C3)C1)C2</smiles>

17

${ }^{13} \mathrm{C}$ NMR

(75 $\mathrm{MHz}, \mathrm{CDCl}_{3}$ )

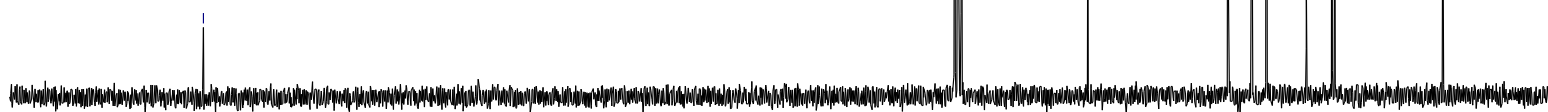

$190-180$

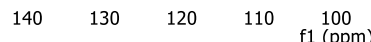
$\stackrel{100}{(\mathrm{ppm})}$
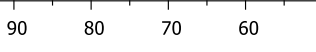

40

10

Figure S39. 1H- and 13C NMR spectra of 17. 

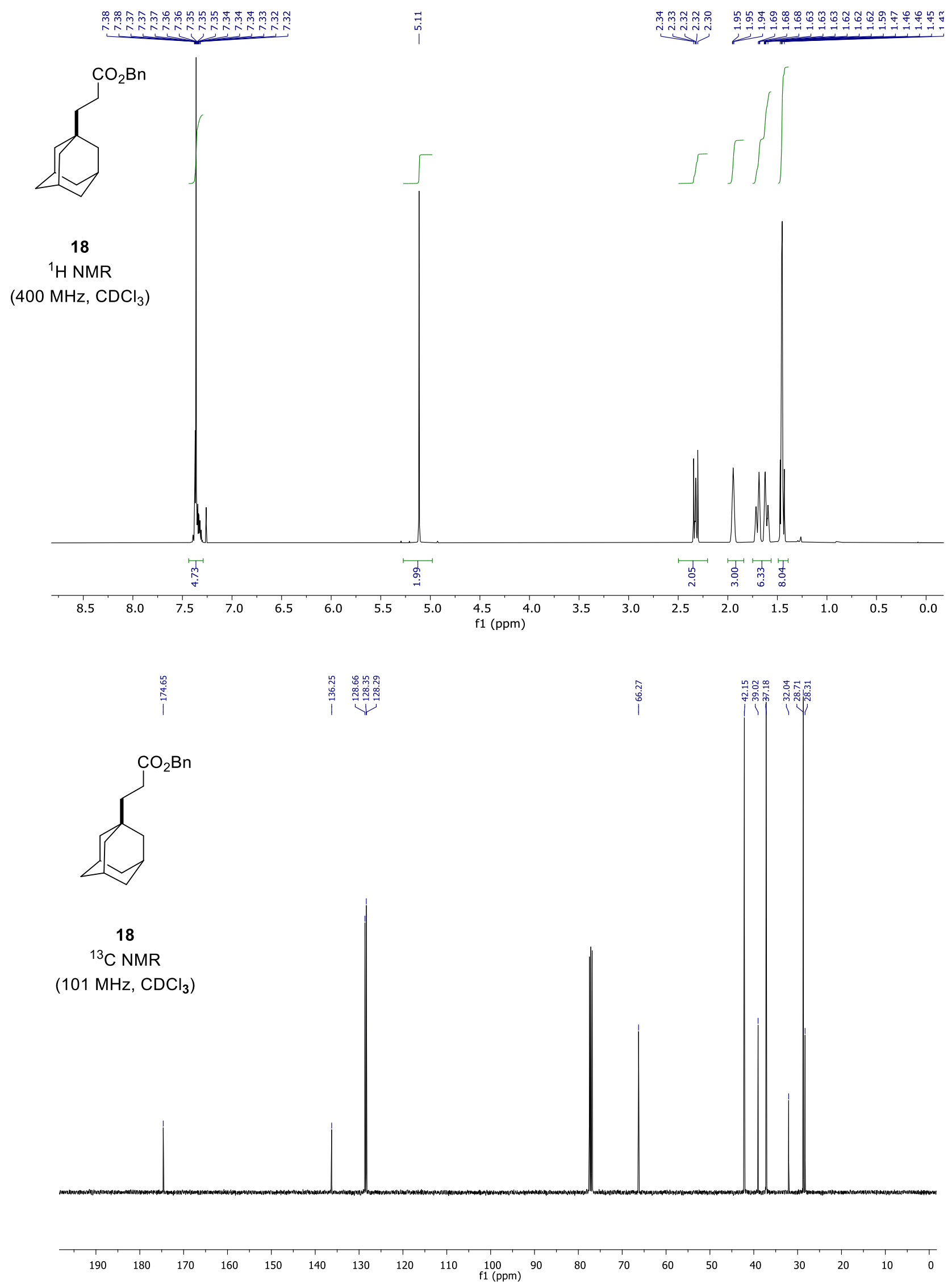

Figure $\mathbf{S 4 0 .} 1 \mathrm{H}$ - and $13 \mathrm{C}$ NMR spectra of 18. 
<smiles>O=C(CCC12CC3CC(CC(C3)C1)C2)Oc1ccccc1</smiles>

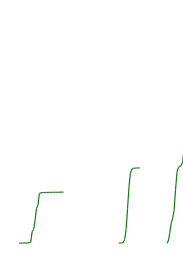

19

${ }^{1} \mathrm{H}$ NMR

$\left(400 \mathrm{MHz}, \mathrm{CDCl}_{3}\right)$

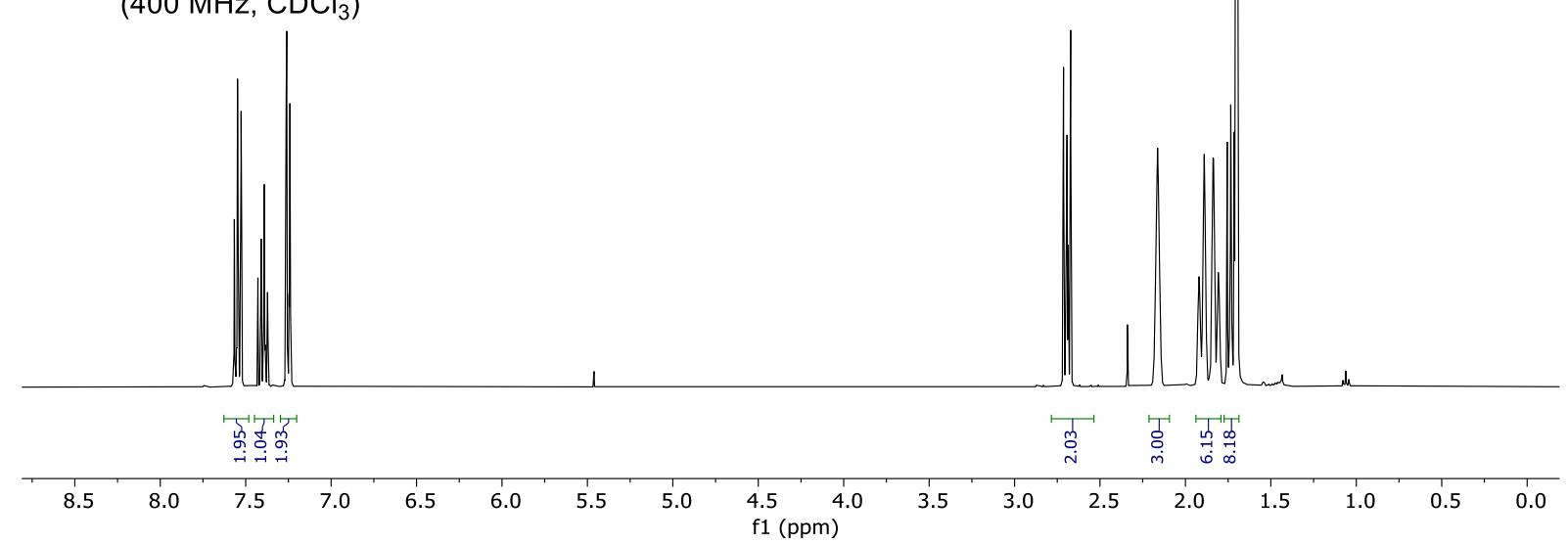<smiles>O=C(CCC12CC3CC(CC(C3)C1)C2)Oc1ccccc1</smiles>

19

${ }^{13} \mathrm{C}$ NMR $\left(101 \mathrm{MHz}, \mathrm{CDCl}_{3}\right.$ )

要

\section{궁ำ}

।

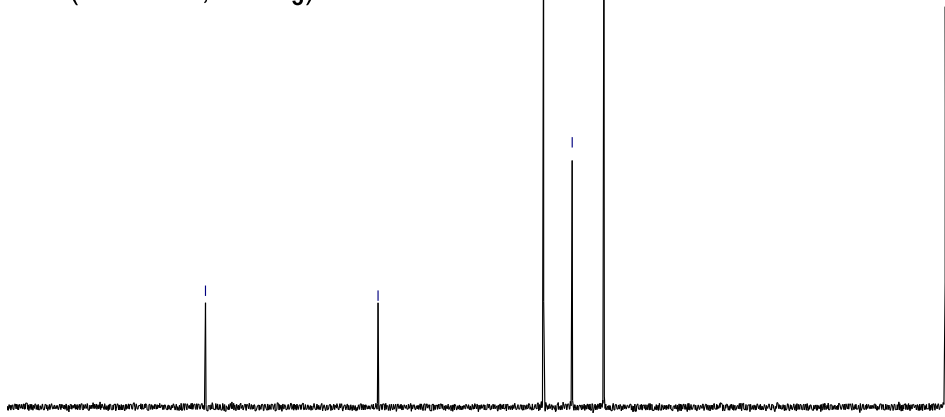

Figure S41. 1H- and 13C NMR spectra of 19. 


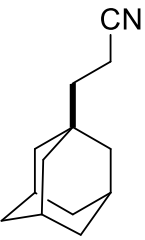

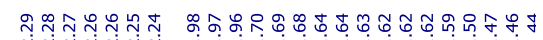

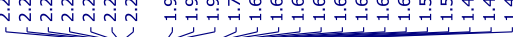

20

${ }^{1} \mathrm{H}$ NMR

(300 MHz, $\mathrm{CDCl}_{3}$ )
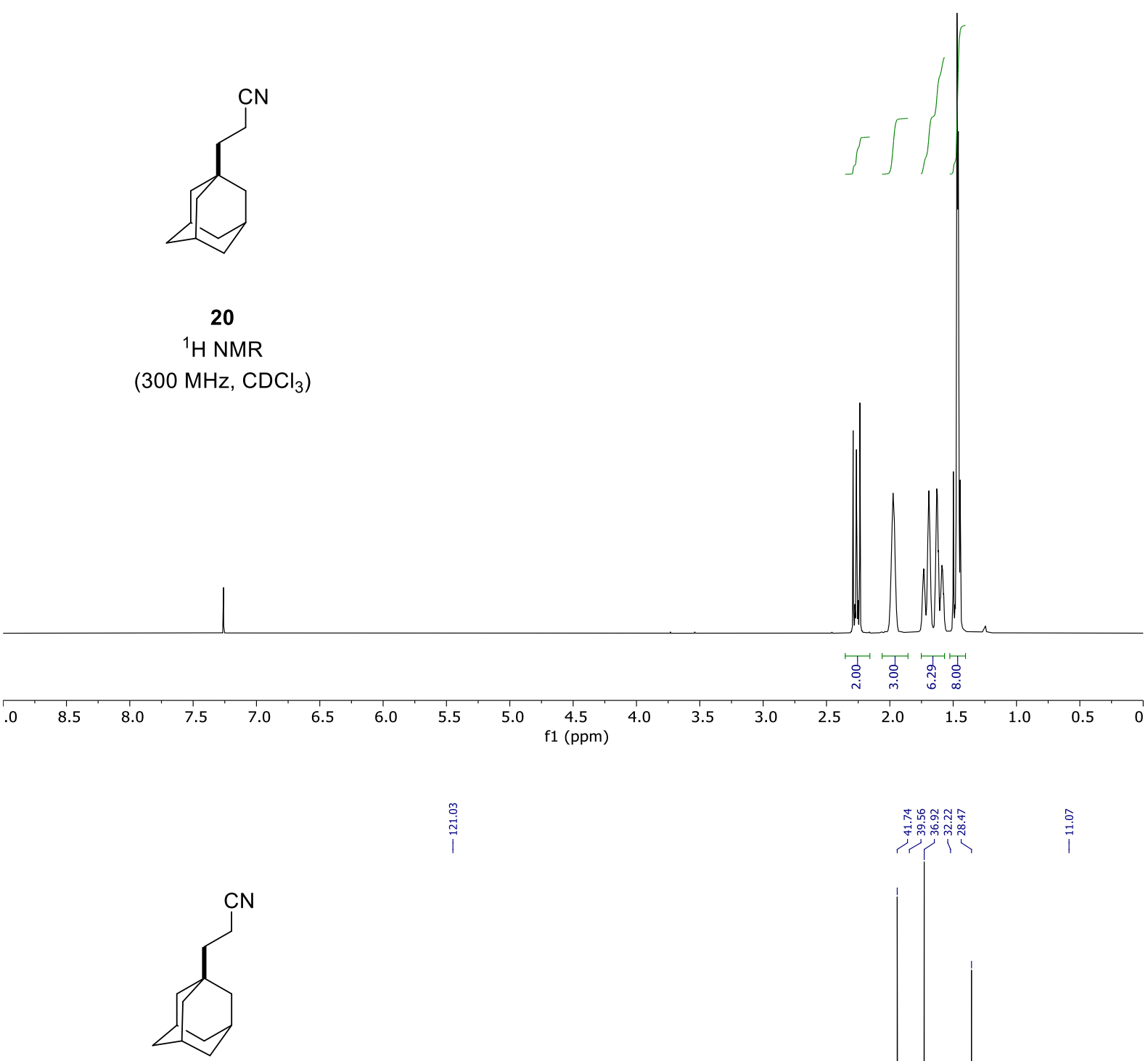

20

${ }^{13} \mathrm{C}$ NMR

(75 $\mathrm{MHz}, \mathrm{CDCl}_{3}$ )
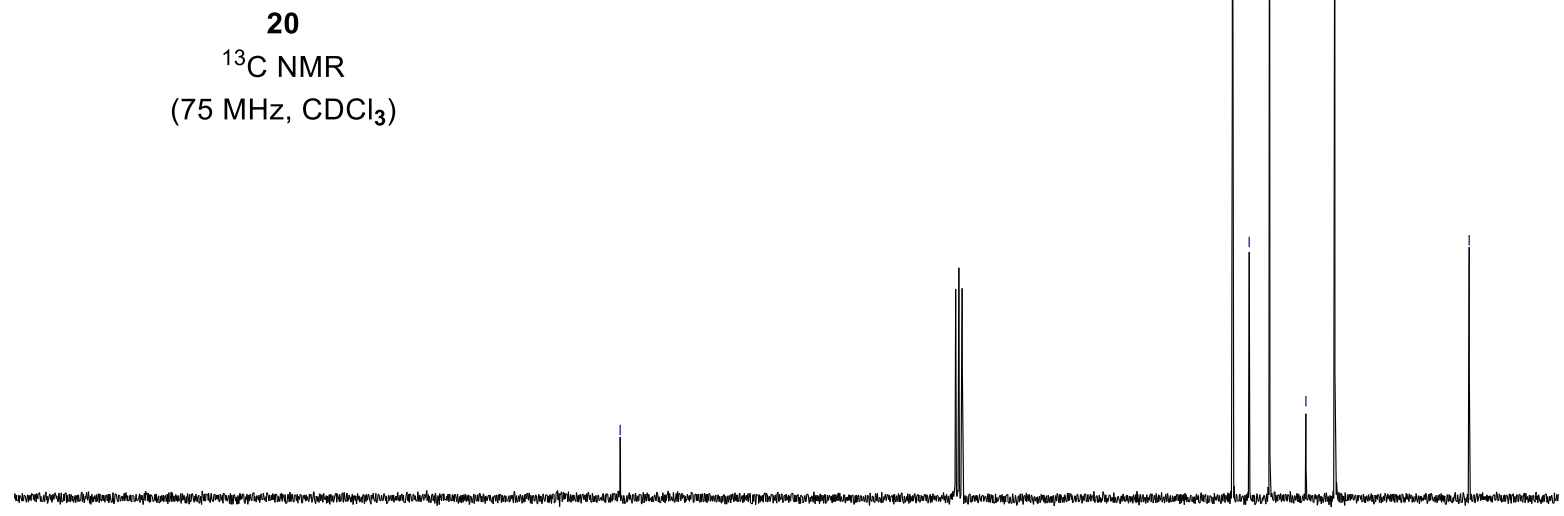

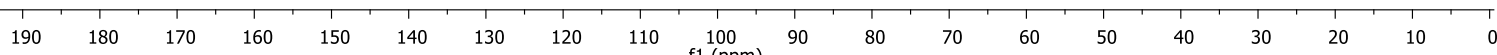

Figure S42. 1H- and 13C NMR spectra of 20. 


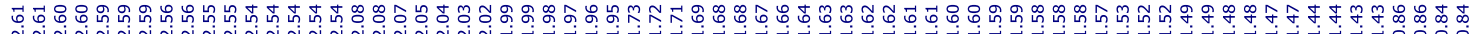
ง<smiles>CCCCC(C)C12CC3CC(CC(C3)C1)C2</smiles>

21

${ }^{1} \mathrm{H}$ NMR

$\left(300 \mathrm{MHz}, \mathrm{CDCl}_{3}\right)$

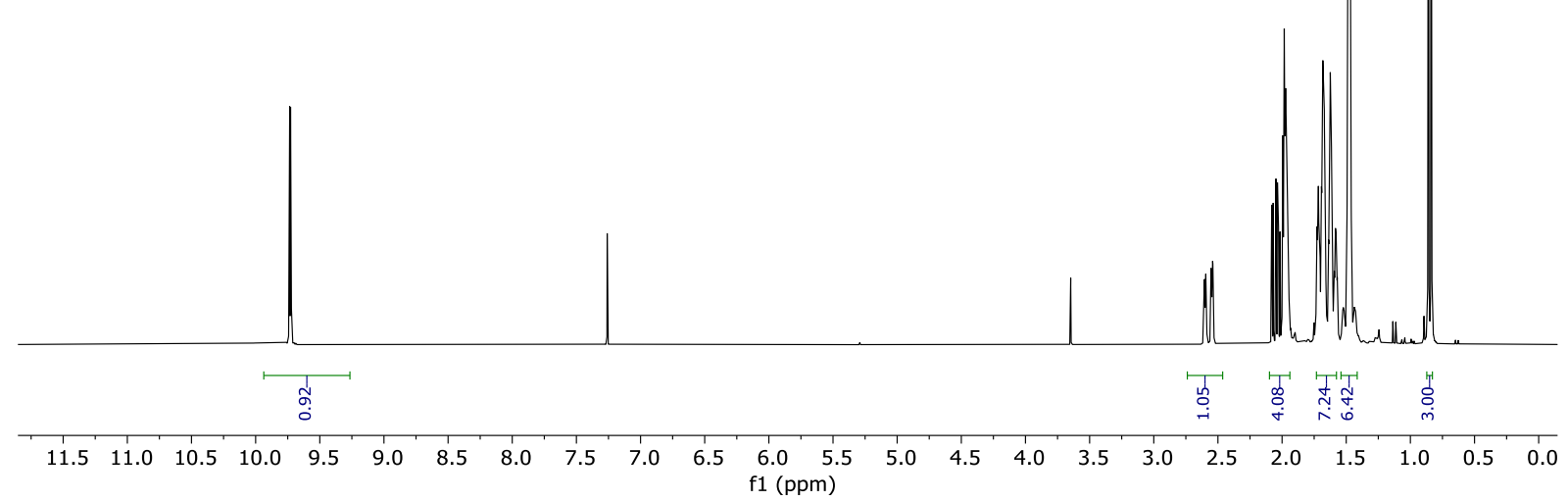<smiles>CC(CC=O)C12CC3CC(CC(C3)C1)C2</smiles>

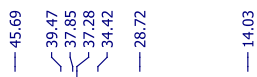

21

${ }^{13} \mathrm{C}$ NMR

$\left(75 \mathrm{MHz}, \mathrm{CDCl}_{3}\right)$

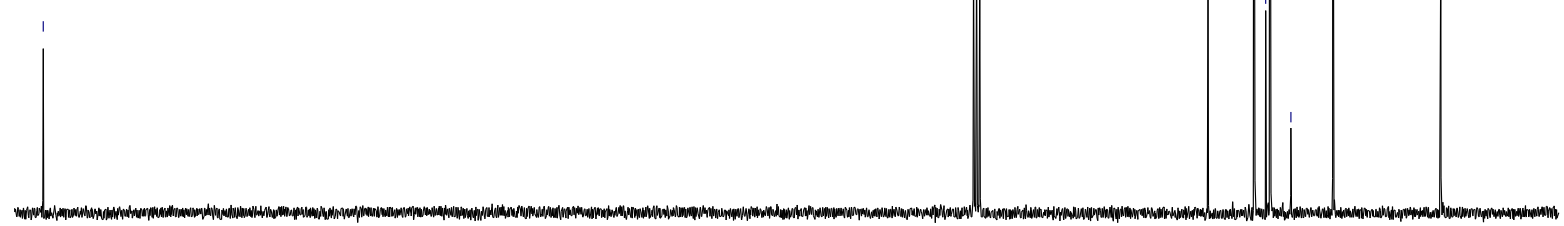

$\begin{array}{llllllllllllllllllllll}200 & 190 & 180 & 170 & 160 & 150 & 140 & 130 & 120 & 110 & 100 & 90 & 80 & 70 & 60 & 50 & 40 & 30 & 20 & 10 & 0\end{array}$

Figure S43. 1H- and 13C NMR spectra of 21. 

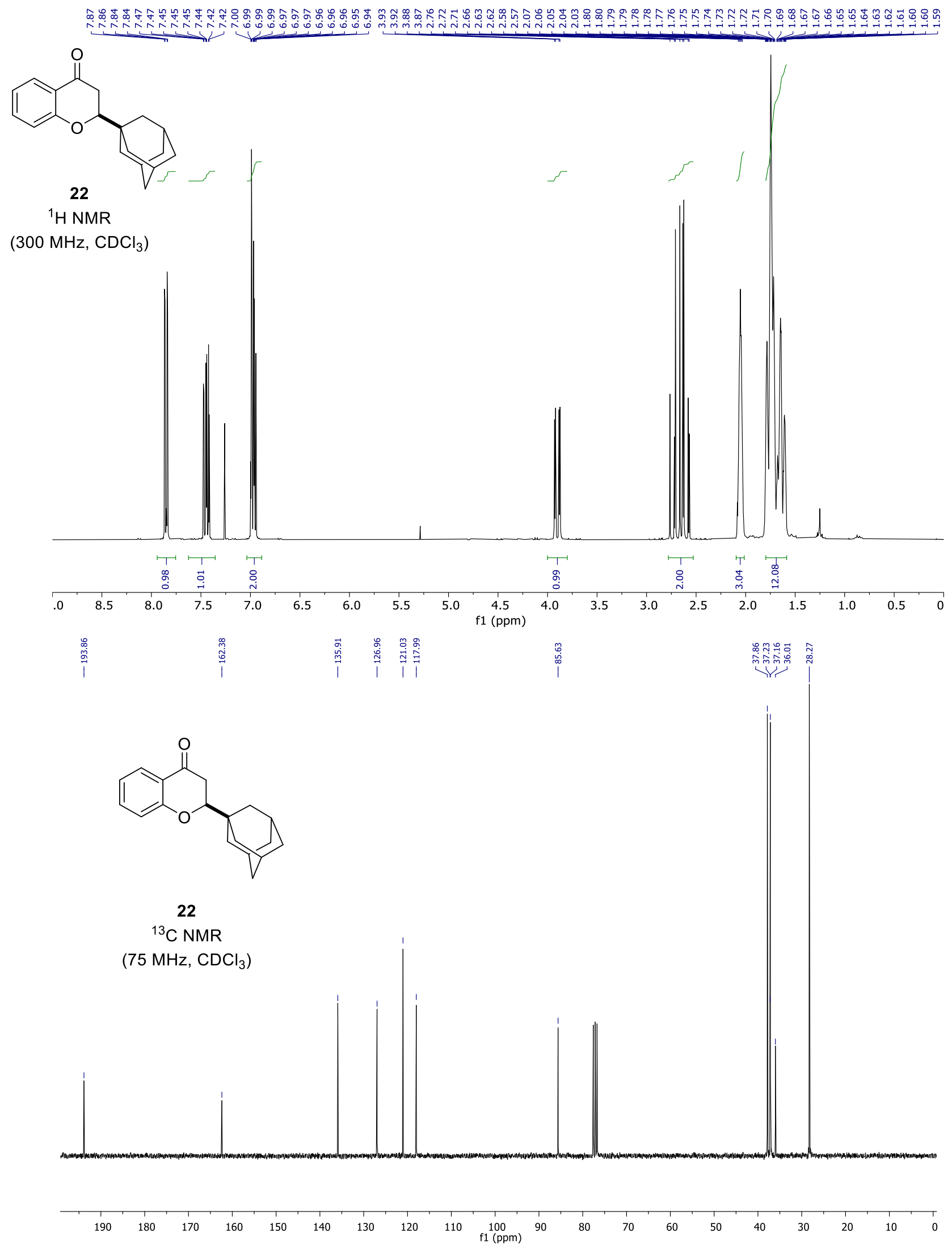

Figure S44. 1H- and 13C NMR spectra of 22. 


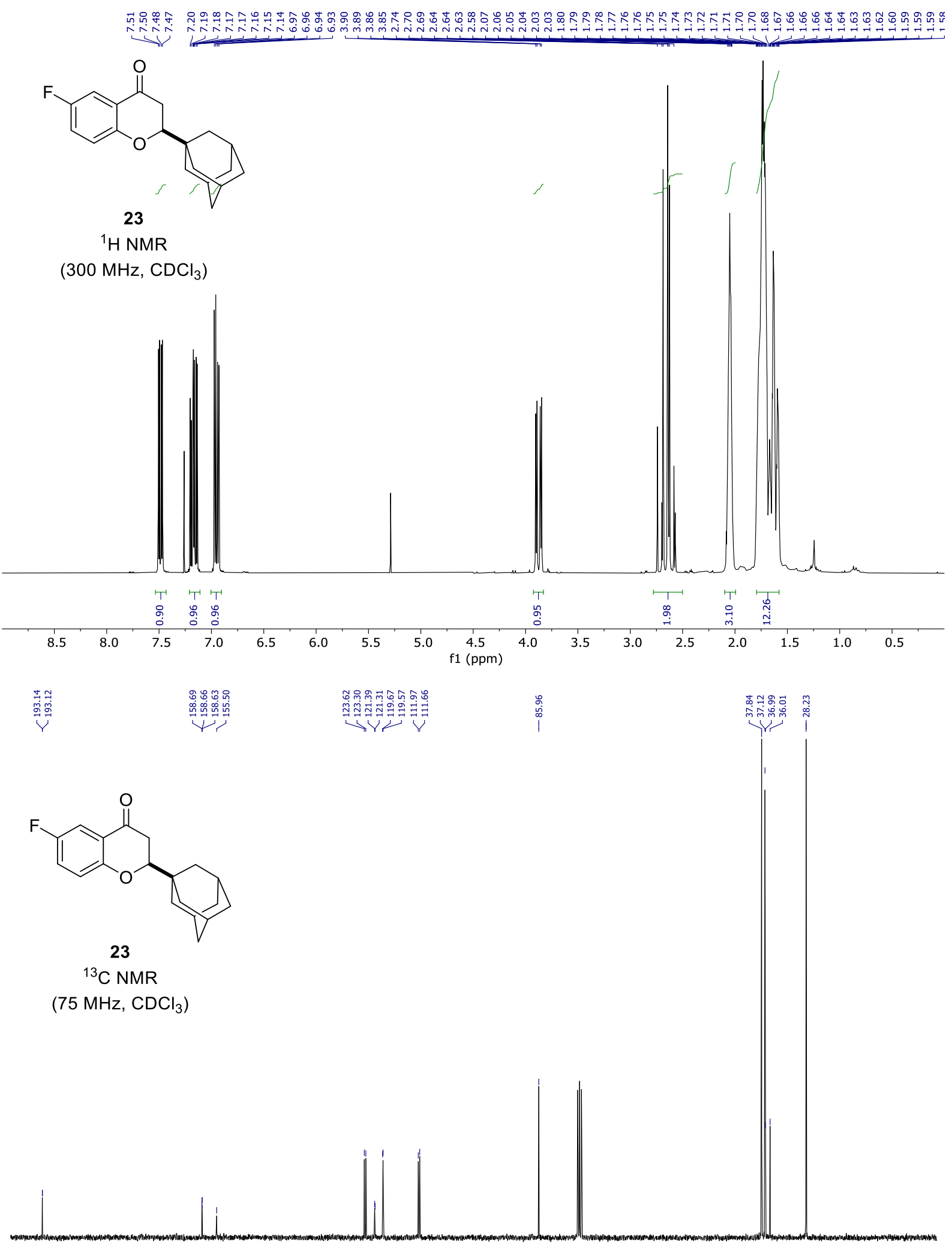

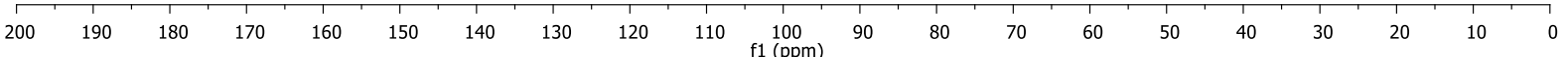

Figure S45. 1H- and 13C NMR spectra of 23. 


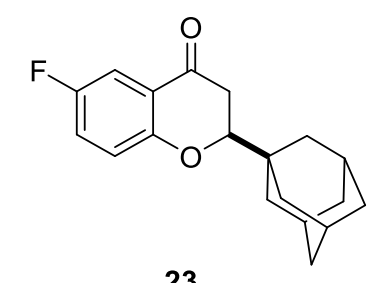

23

${ }^{19} \mathrm{~F}$ NMR

(282 $\mathrm{MHz}, \mathrm{CDCl}_{3}$ )

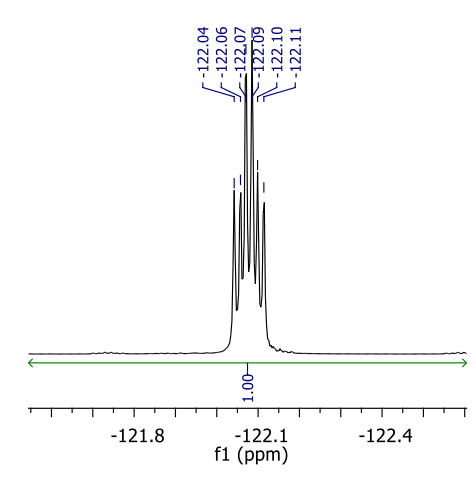

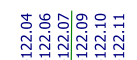

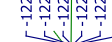

4

$\stackrel{8}{\circ}$
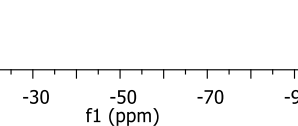

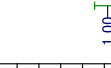

\section{-}

Figure S46. 19F NMR spectra of 23. 


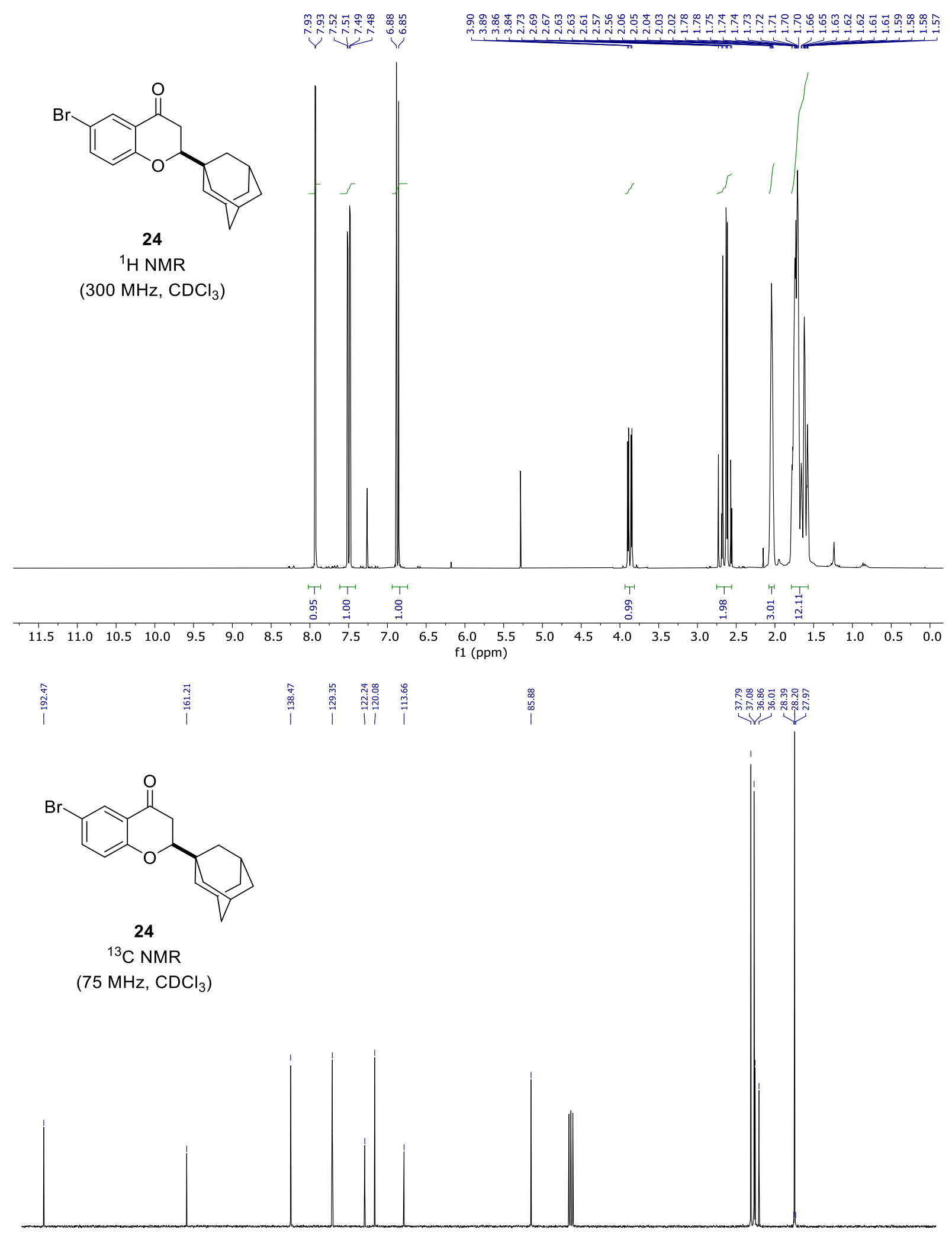

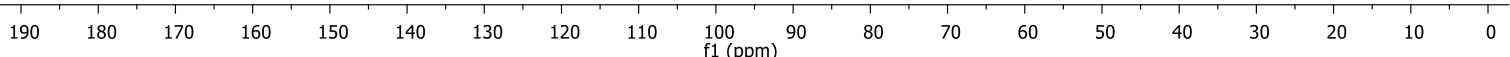

Figure S47. 1H- and 13C NMR spectra of 24. 


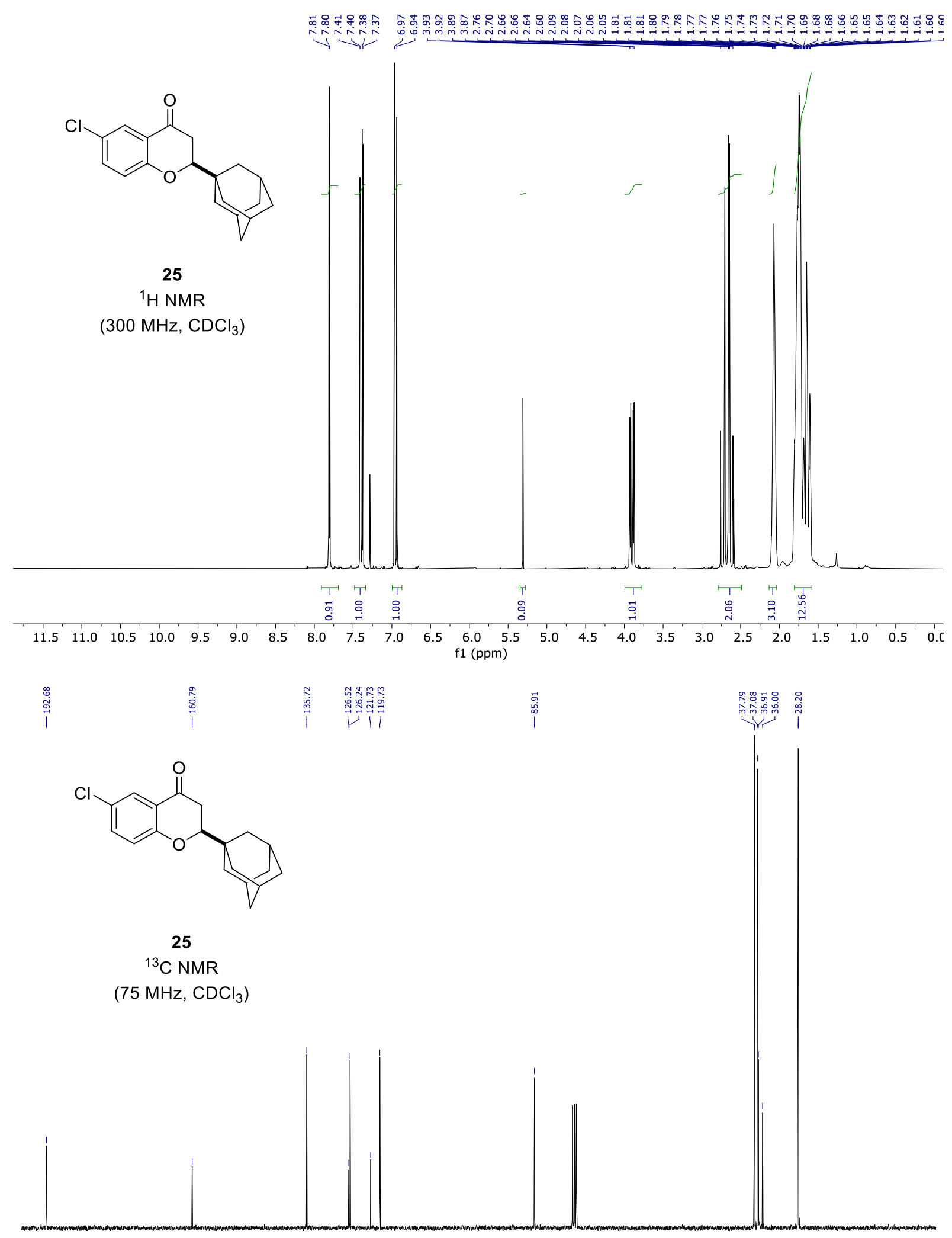

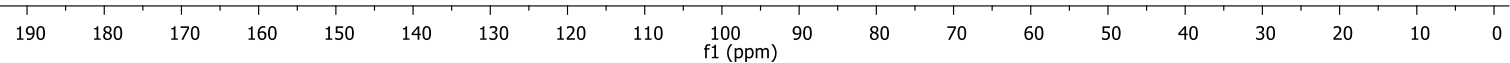

Figure S48. $1 \mathrm{H}$ - and $13 \mathrm{C}$ NMR spectra of $\mathbf{2 5 .}$ 


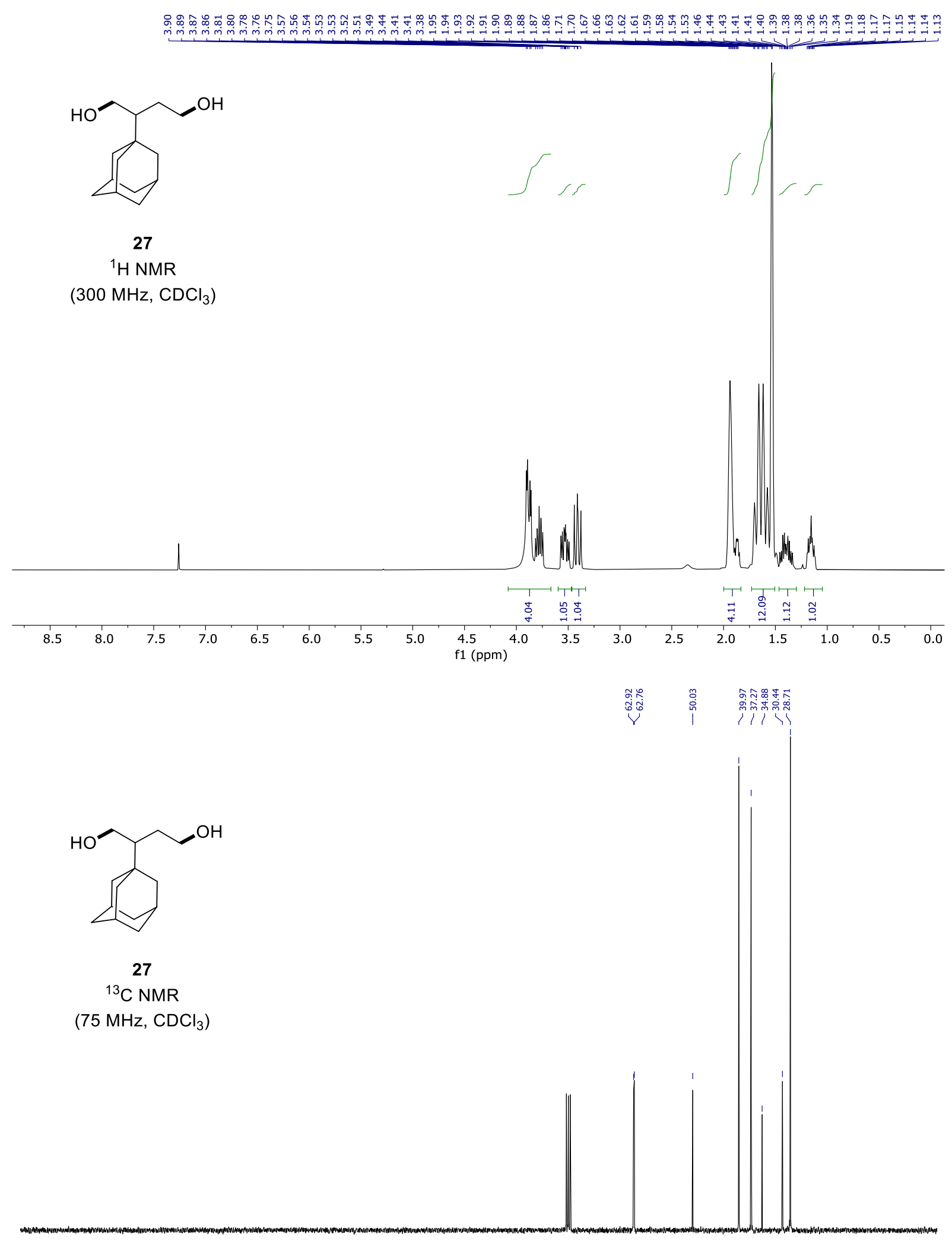

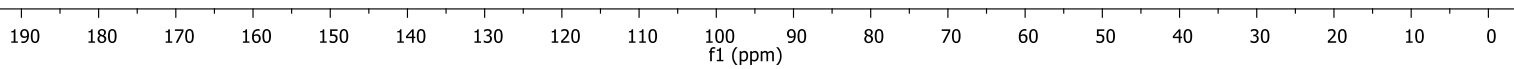

Figure S49. 1H- and 13C NMR spectra of 27. 


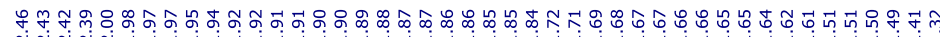<smiles>CC1(C)OC(=O)CC1C12CC3CC(CC(C3)C1)C2</smiles>

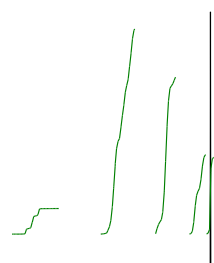

28

${ }^{1} \mathrm{H}$ NMR

$\left(300 \mathrm{MHz}, \mathrm{CDCl}_{3}\right)$

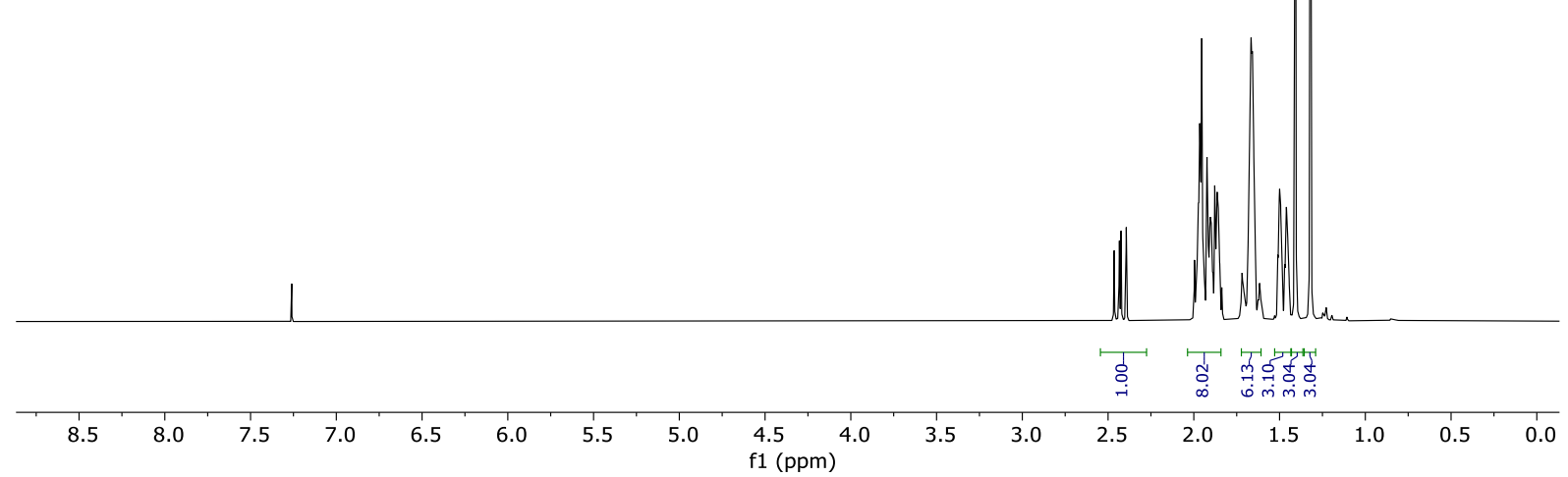

<smiles>CC1(C)OC(=O)CC1C12CC3CC(CC(C3)C1)C2</smiles>

28

${ }^{13} \mathrm{C}$ NMR

$\left(75 \mathrm{MHz}, \mathrm{CDCl}_{3}\right)$

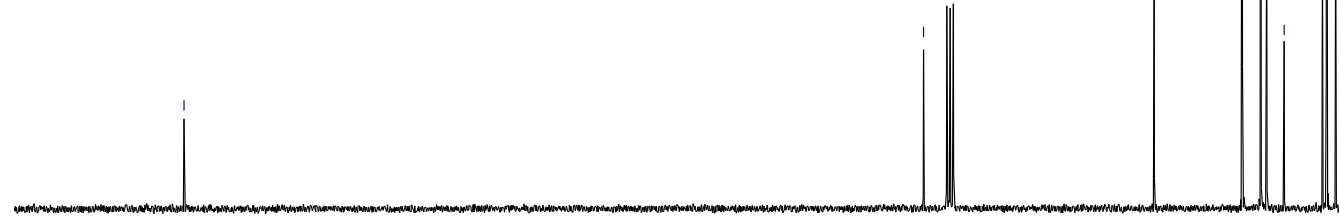

लiㅔ

परी

$\begin{array}{llllllllll}190 & 180 & 170 & 160 & 150 & 140 & 130 & 120 & 110 & \underset{f 1(\mathrm{ppm})}{100}\end{array}$

$\begin{array}{lllll}100 & 90 & 80 & 70 & 60\end{array}$

Figure S50. 1H- and 13C NMR spectra of 28. 


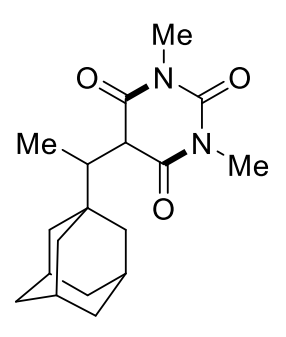

29

${ }^{1} \mathrm{H}$ NMR (300 MHz, $\mathrm{CDCl}_{3}$ )
추 ㅇํำกิ

mi mंm

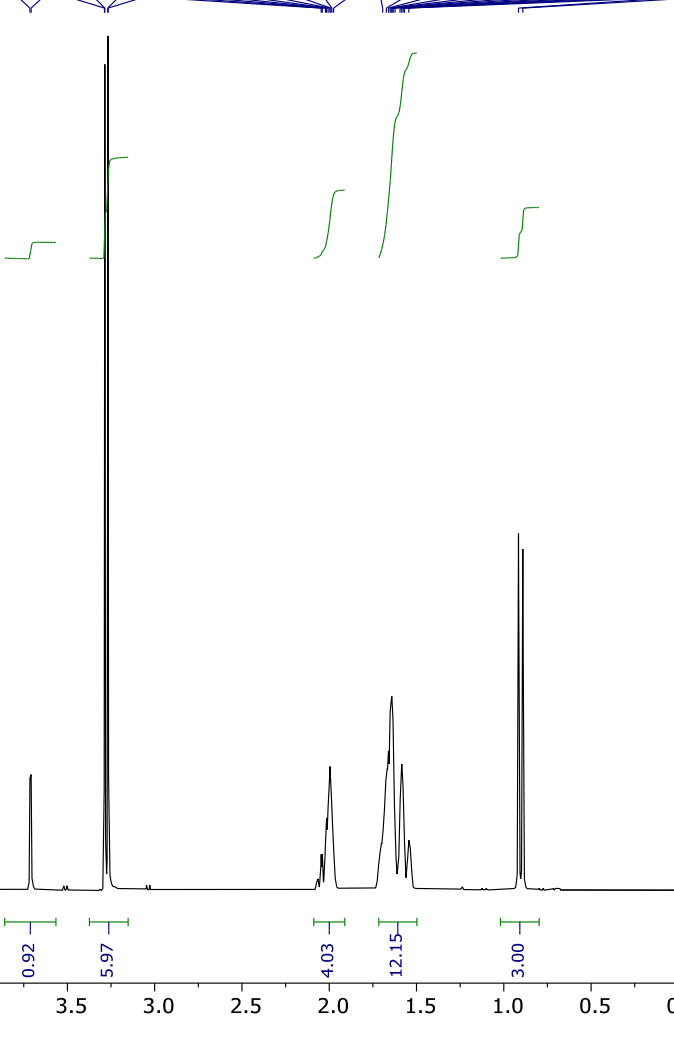<smiles>CC(C1C(=O)N(C)C(=O)N(C)C1=O)C12CC3CC(CC(C3)C1)C2</smiles>

29

${ }^{13} \mathrm{C}$ NMR (75 $\mathrm{MHz}, \mathrm{CDCl}_{3}$ )
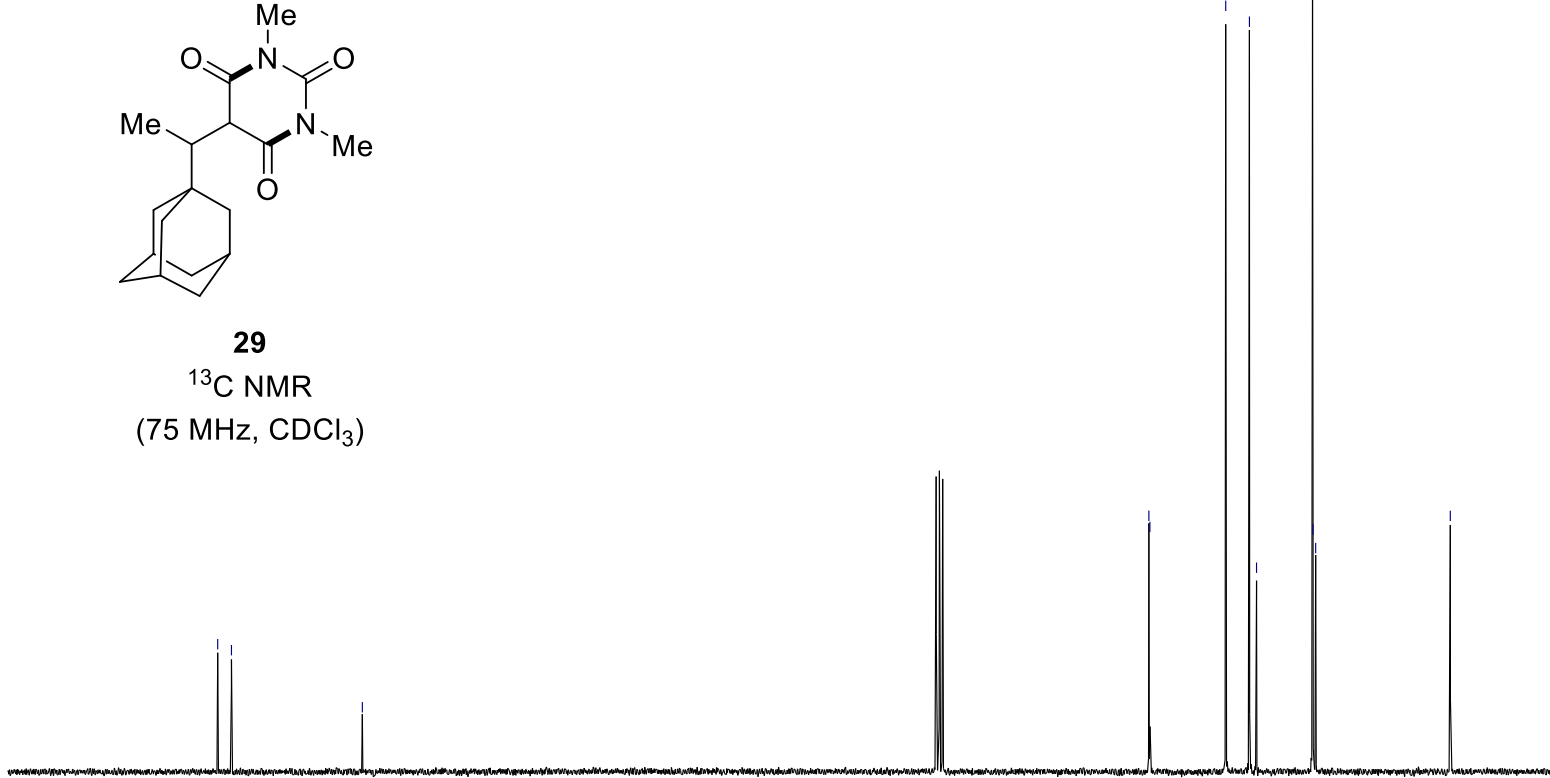

Figure S51. 1H- and 13C NMR spectra of 29. 


\section{Mechanistic experiments:}

\section{1- Deuterium incorporation experiments.}

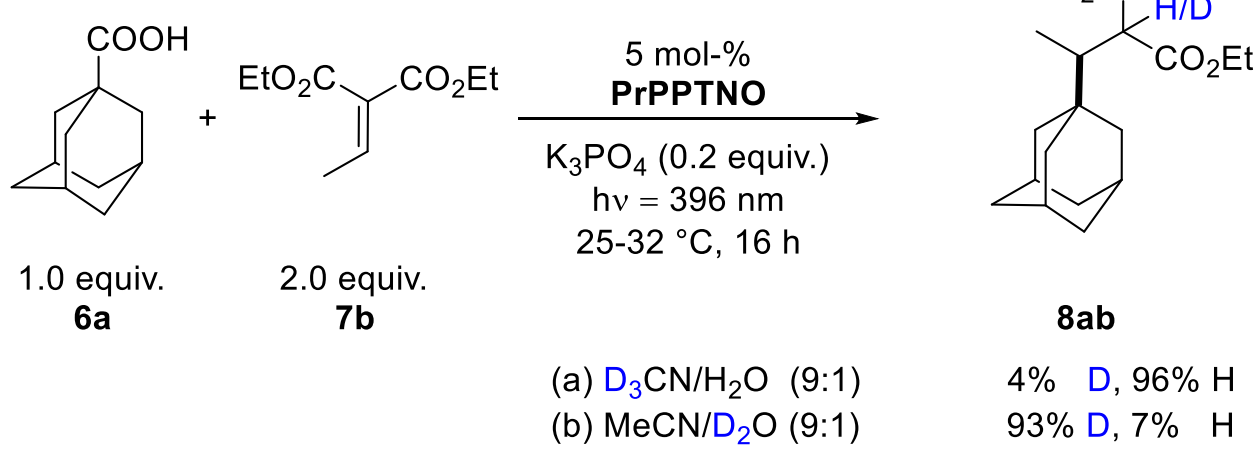

Scheme S1. Deuterium incorporation experiments of the reaction of $\mathbf{6 a}$ and $\mathbf{7 b}$.

Reactions was performed under Schlenk conditions using a $5 \mathrm{ml}$ process vial equipped with a NS 14.5 rubber septum. To this vial was added 1-adamantanecarboxylic acid (45.1 mg, $0.25 \mathrm{mmol}, 1.0$ equiv.), photocatalyst XX (5.4 mg, $5.0 \mathrm{~mol} \%)$ and $\mathrm{K}_{3} \mathrm{PO}_{4}(10.6 \mathrm{mg}, 20 \mathrm{~mol}-\%)$. Next, the vial was evacuated and purged with argon for 3 times. Diethyl ethylidenmalonate (93.1 mg, $0.5 \mathrm{mmol}, 2.0$ equiv.) was then added to the flask under argon. Afterwards, $5 \mathrm{ml}$ of (a) $\mathrm{D}_{3} \mathrm{CN}: \mathrm{H}_{2} \mathrm{O}(9: 1)$ or (b) $\mathrm{MeCN}: \mathrm{D}_{2} \mathrm{O}(9: 1)$ were added and the reaction mixture was stirred for 2 minutes in the dark. The reaction mixture was then irradiated for $16 \mathrm{~h}$ and quenched afterwards upon exposure to air and bubbling air through the solution using a pipette. The reaction was concentrated under reduced pressure and the crude product was purified by column chromatography ( $n$-pentane:EtOAc $=20: 1)$ yielding the title compound $\mathbf{X X}$ as a colorless oil. 
<smiles>CCOC(=O)C(C)(CC)C(C)C12CC3CC(CC(C3)C1)C2</smiles>

$8 \mathbf{a b}$

${ }^{1} \mathrm{H}$ NMR

$\left(300 \mathrm{MHz}, \mathrm{CDCl}_{3}\right)$
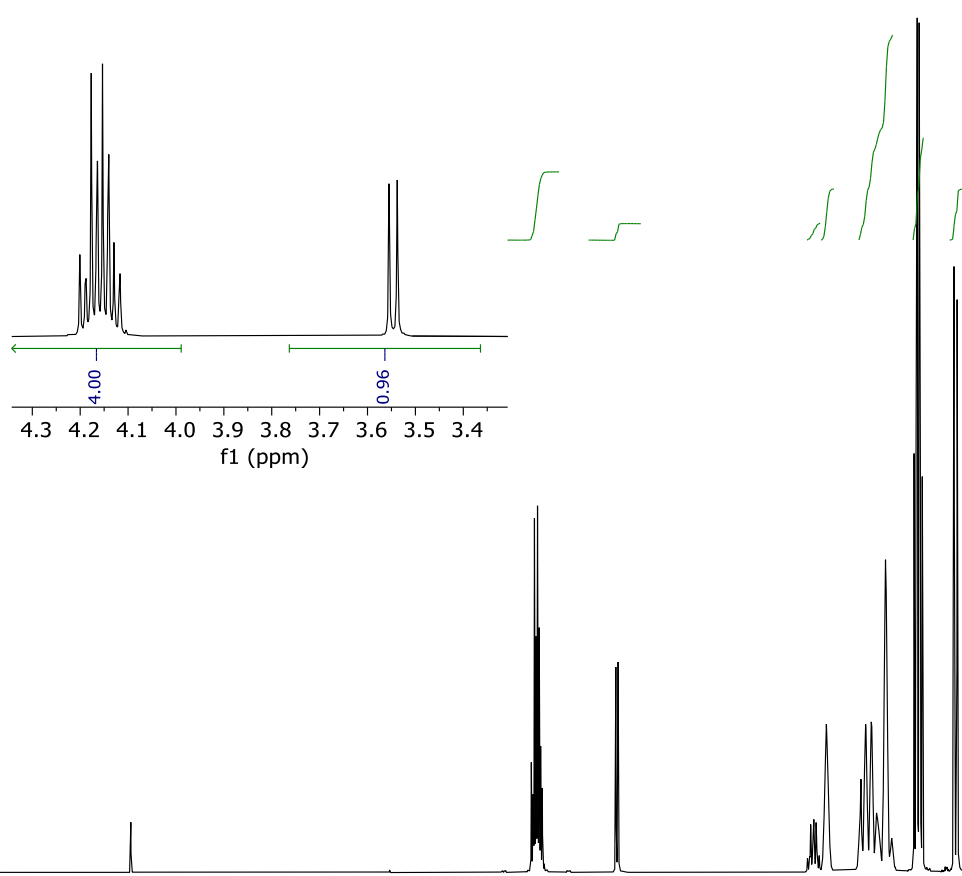

(a) $\mathrm{D}_{3} \mathrm{CN} / \mathrm{H}_{2} \mathrm{O}(9: 1)$

$4 \% \mathrm{D}, \mathbf{9 6 \%} \mathrm{H}$

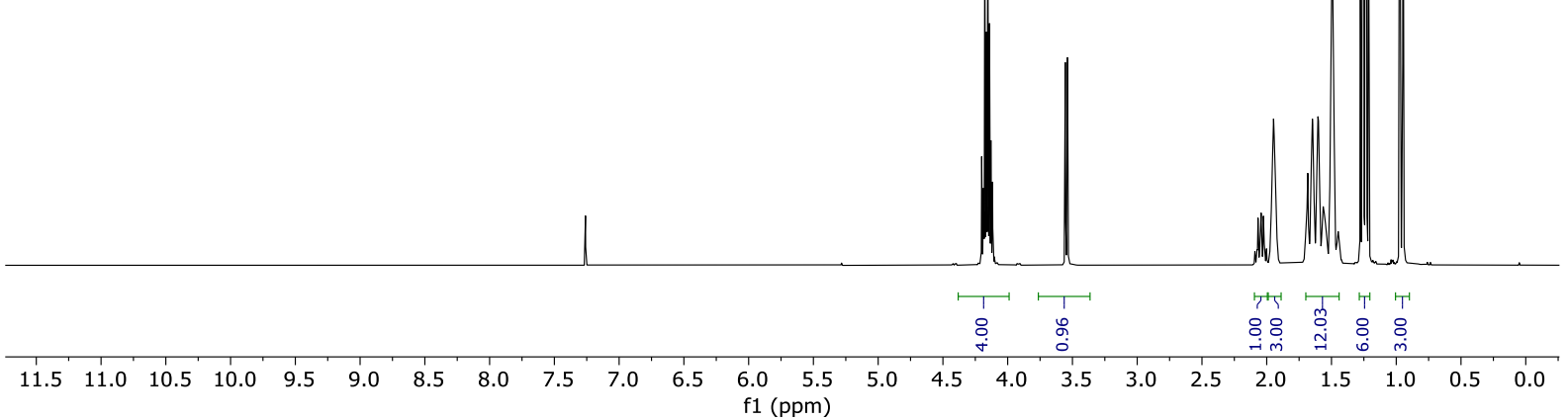

Figure S52. 1H NMR spectrum of 8ab from reaction (a).

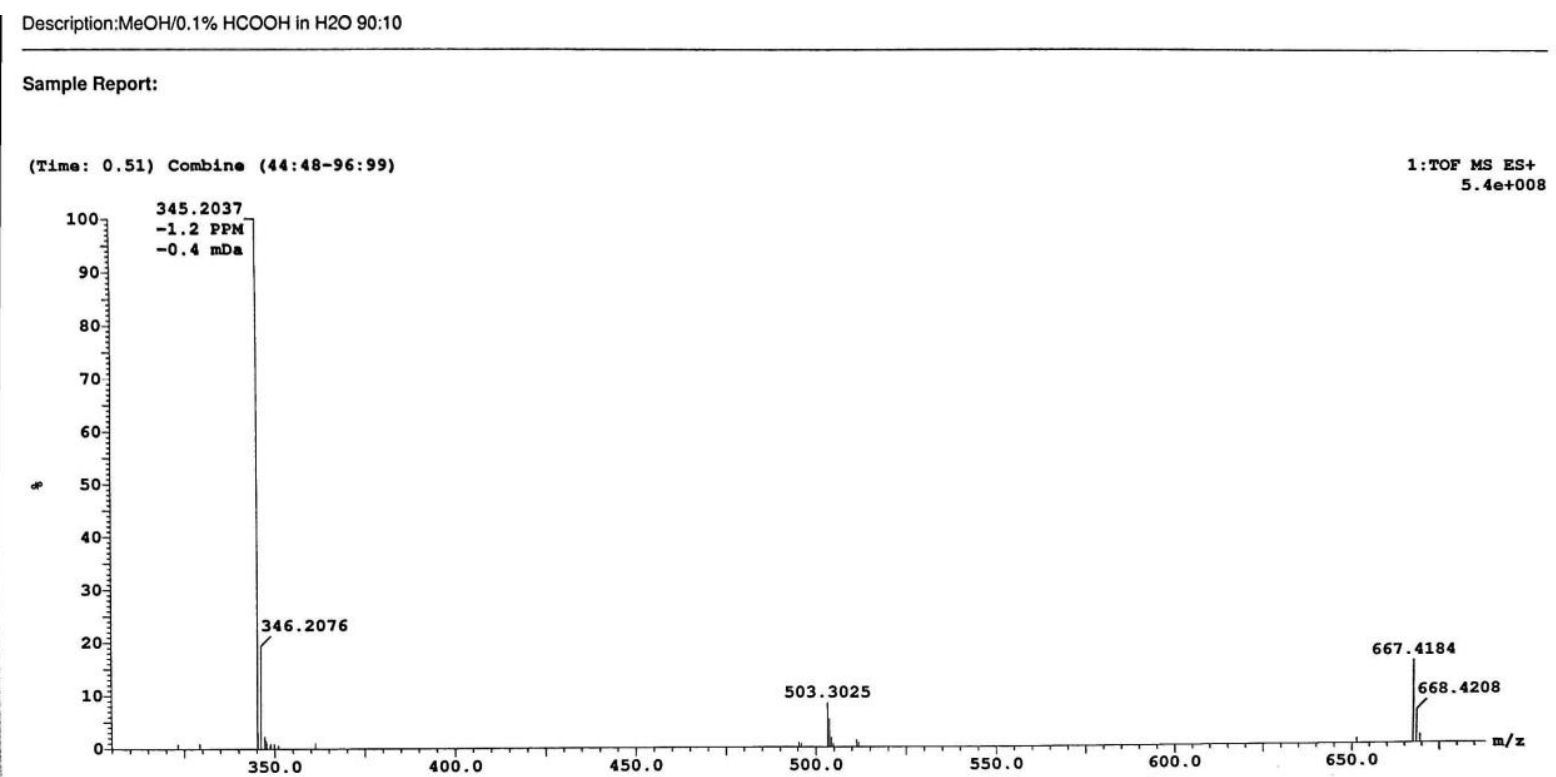

Figure S53. HRMS spectrum of the reaction mixture of reaction (a). 


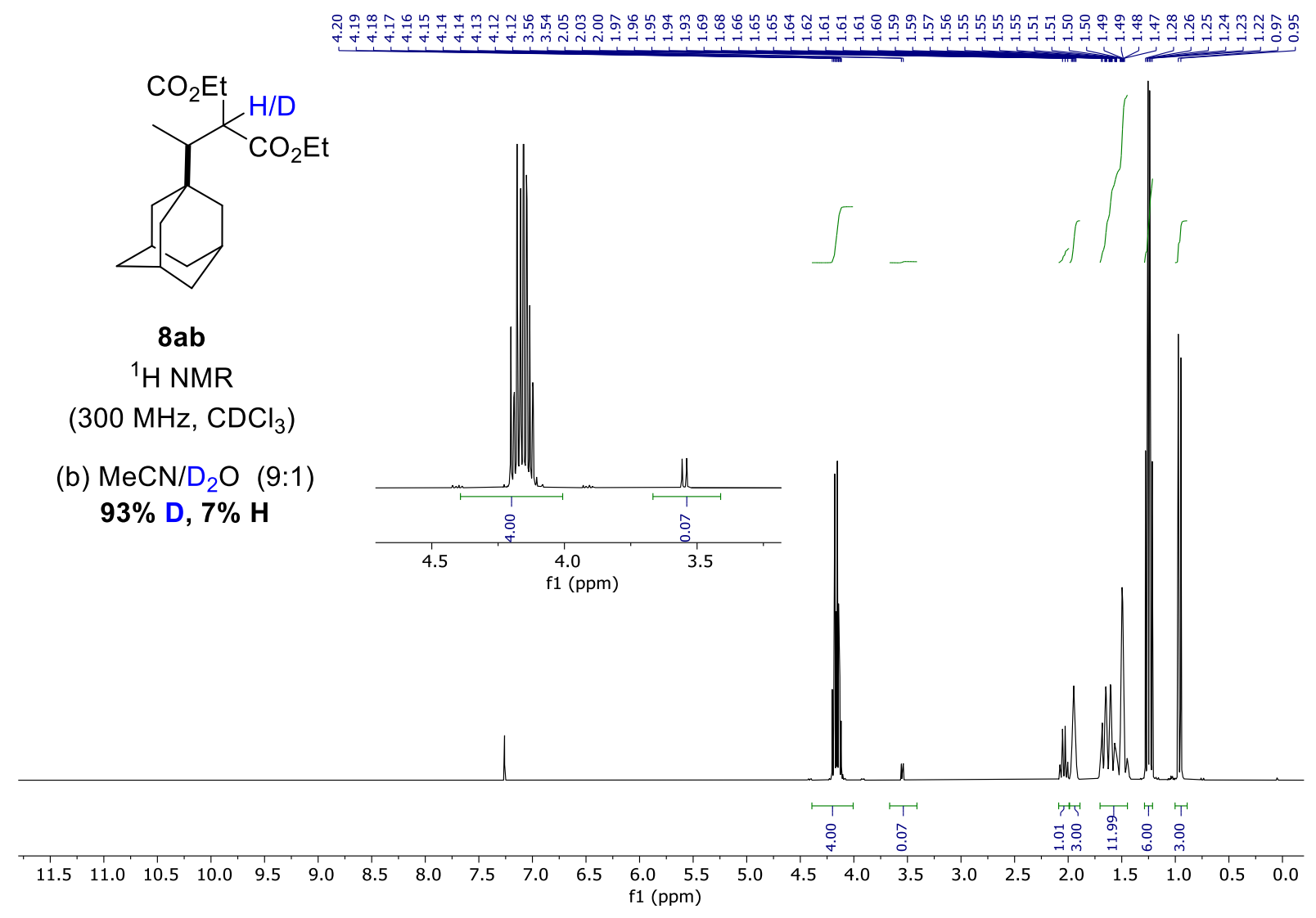

Figure S54. 1H NMR spectrum of 8ab from reaction (b). 


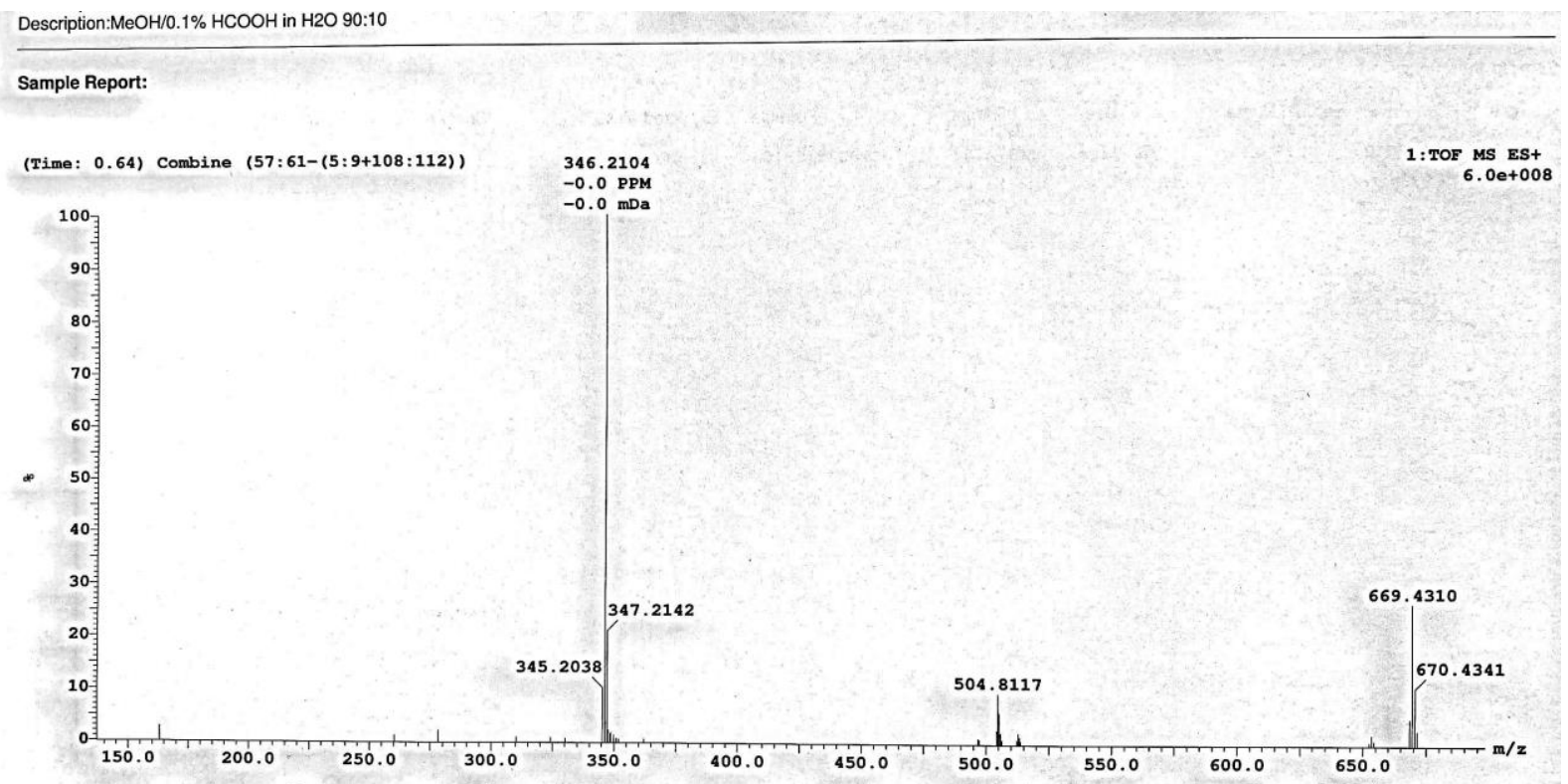

Figure S55. HRMS spectrum of the reaction mixture of reaction conditions (b).

\section{2- TEMPO quenching experiment:}

TEMPO

(1.25 equiv.)<smiles>CC=C(C(=O)OCC)C(=O)OCC</smiles>

1.0 equiv. $6 a$
2.0 equiv.

$7 \mathrm{~b}$

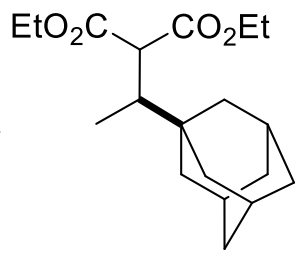

$8 a b$ not detected

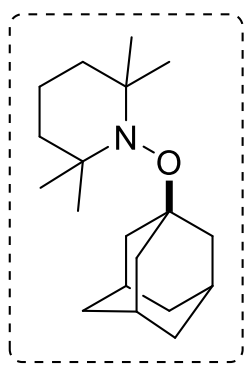

26

detected by

LC-MS

Scheme S2. TEMPO radical quenching experiment of the reaction of $\mathbf{6 a}$ and $\mathbf{7 b}$.

TEMPO quenching experiment was performed under Schlenk conditions using a $5 \mathrm{ml}$ process vial equipped with a NS 14.5 rubber septum. To this vial was added 1-adamantanecarboxylic acid carboxylic acid (90.1 mg, $0.5 \mathrm{mmol}, 1.0$ equiv.), photocatalyst XX (10.8 mg, $5.0 \mathrm{~mol} \%), 2,2,6,6-$ Tetramethylpiperidin-1-yl)oxyl (97.7 mg, 0.625 mmol, 1.25 equiv.) and $\mathrm{K}_{3} \mathrm{PO}_{4}$ (21.2 mg, $0.1 \mathrm{mmol}$, 20 mol-\%). Next, the vial was evacuated and purged with argon for 3 times followed by the addition of Diethyl ethylidenmalonate ( $93.1 \mathrm{mg}, 0.5 \mathrm{mmol}, 2.0$ equiv.) under argon. Afterwards, $4.5 \mathrm{ml}$ of $\mathrm{MeCN}$ and $0.5 \mathrm{ml}$ degassed $\mathrm{H}_{2} \mathrm{O}$ were added and the reaction mixture was irradiated for $16 \mathrm{~h}$. Subsequently, $0.5 \mathrm{ml}$ sample was taken and measured by LC-MS (see LC-MS spectrum). 


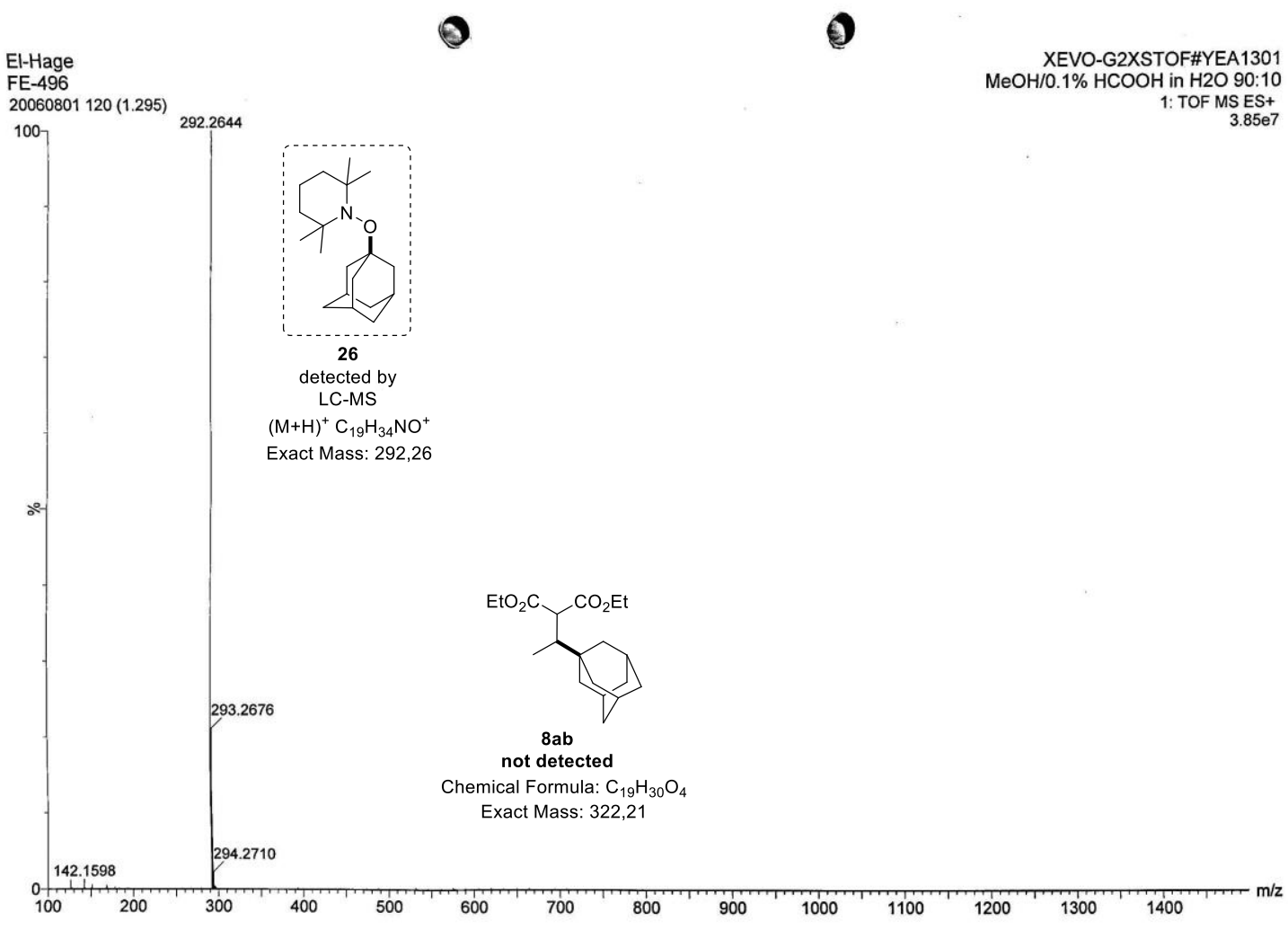

Figure S56. LC-MS spectrum of the TEMPO quenching experiment. 


\section{Crystallographic Data}

Single crystal was grown from dichloromethane by solvent evaporation. Data were collected on a Bruker Kappa APEX II Duo diffractometer using Mo-K $\alpha$ radiation. The structures were solved by direct methods (SHELXS-97: Sheldrick, G. M. Acta Cryst. 2008, A64, 112.) and refined by full-matrix least-squares procedures on $F^{2}$ (SHELXL-2014 and SHELXL-2018, resp.: Sheldrick, G. M. Acta Cryst. 2015, C71, 3.). XP (Bruker axs) was used for graphical representations.

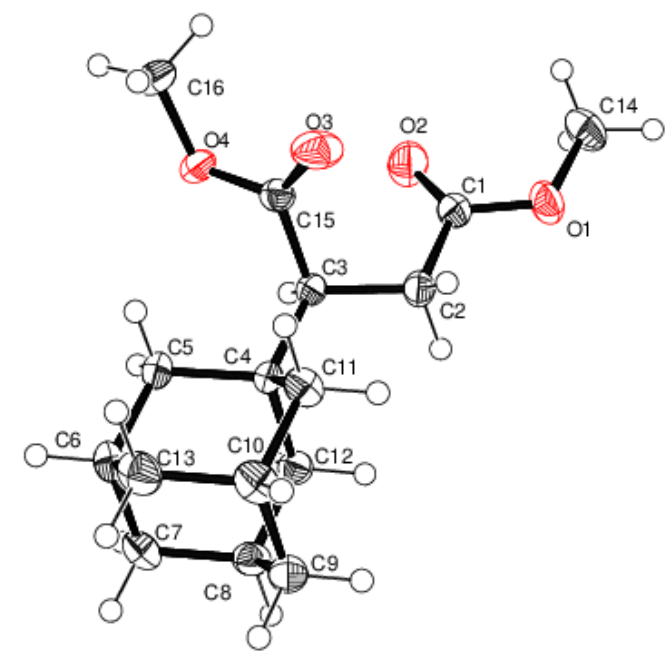

Figure S57. X-ray crystal structure of product 8ab. Displacement ellipsoids correspond to $30 \%$ probability. Hydrogen atoms are omitted for clarity.

Table S6. Crystallographic details of 8ab.

\begin{tabular}{|c|c|}
\hline chemical formula & $\mathrm{C}_{16} \mathrm{H}_{24} \mathrm{O}_{4}$ \\
\hline formula weight & 280.35 \\
\hline crystal system & monoclinic \\
\hline \multicolumn{2}{|l|}{ unit cell dimensions } \\
\hline$a[\AA ̊]$ & $9.3426(9)$ \\
\hline$b[\AA]$ & $21.642(2)$ \\
\hline$c[\AA]]$ & $7.3217(7)$ \\
\hline$\alpha[\operatorname{deg}]$ & 90 \\
\hline$\beta[\operatorname{deg}]$ & $95.5215(19)$ \\
\hline$\gamma[\operatorname{deg}]$ & 90 \\
\hline$V\left[\AA^{3}\right]$ & $1473.5(2)$ \\
\hline$T[\mathbf{K}]$ & $150(2)$ \\
\hline space group & $P 2_{1} / c$ \\
\hline
\end{tabular}


Z

$\mu\left[\mathrm{mm}^{-1}\right]$

density $\left[\mathrm{g} / \mathrm{cm}^{3}\right]$

no. of reflections measured

no. of independent reflections

no. of observed reflections $(I>2 \sigma(I))$

no. of parameters

$R_{1}(I>2 \sigma(I))$

$w R_{2}$ (all data)

Goodness of fit on $F^{2}$

largest diff. peak and hole [e/ $\left./ \AA^{3}\right]$
4

0.09

1.264

27916

$3562\left(R_{\text {int }}=0.028\right)$

2926

183

0.041

0.116

1.03

0.36 and -0.18

\section{References}

1. Kovalenko, V. N.; Kulinkovich, O. G., The resolution of trans-2,2-dichloro-3methylcyclopropanecarboxylic acid via crystallization of its salts with $(+)-$ and $(-)-\alpha-$ phenylethylamine, and the transformation of the resulting enantiomers into (R)- and (S)-dimethyl 2methylsuccinates. Tetrahedron: Asymmetry 2011, 22, 26-30.

2. Dickey, J. B.; Gray, A. R., Barbituric acid. Org Synth 1938, 18, 8-9. 